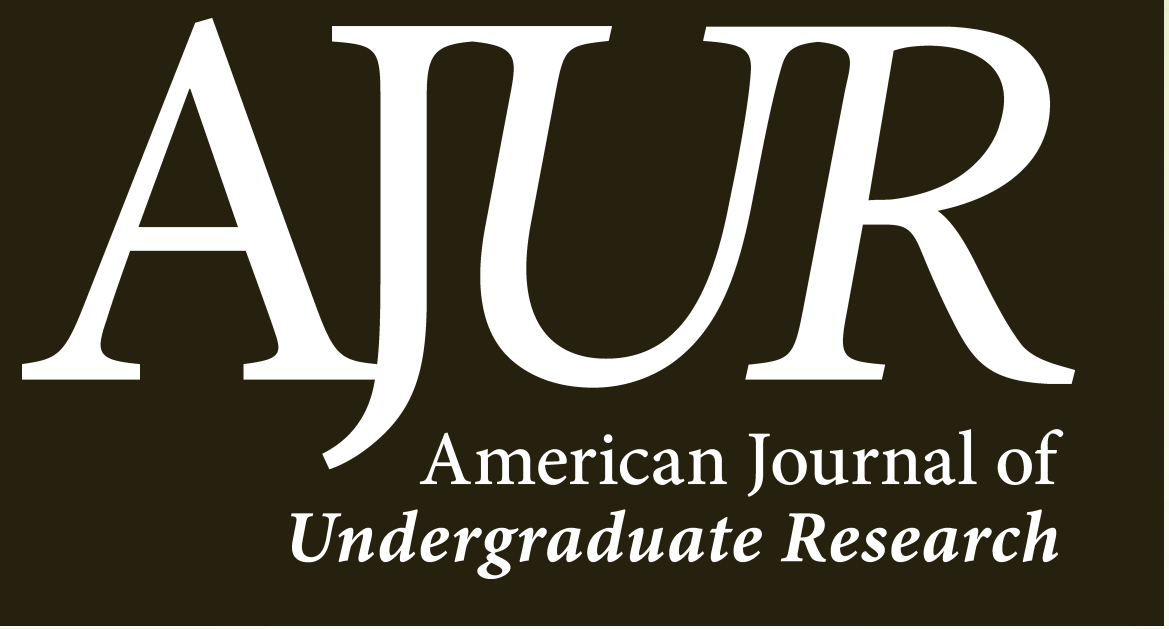

Volume 17 | Issue 3 | December 2020

www.ajuronline.org 


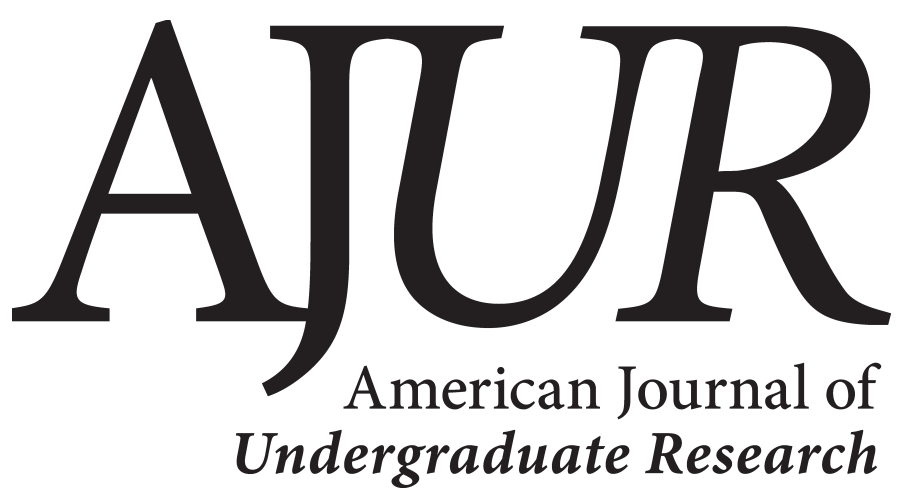

Volume 17 | Issue 3 | December 2020

www.ajuronline.org

2

3

11

AJUR History and Editorial Board

Investigating the Effect of Flock Size on Vigilance in the American Coot (Fulica americana) in Relationship to Habitat

Dat Q. Lam, Suyash P. Rizal, Roxanne Cota, Miguel Sicaja, Gabriel Cox, Brandon Wakefield,

\& Zia Nisani

Cyclophosphamide Depletes Ovarian Follicles in Mice During Both the Light and Dark Phases of the Circadian Cycle Benjamin Z. Koch \& Kristen A. Roosa

Associations of Maternal Controlling Feeding Practices with Child Internalizing Symptoms and Body Mass Index in EthnicallyDiverse Mother-Child Dyads

Paulina Mozdzierz, Genevieve F. Dunton, \& Tyler B. Mason

Synthesis of Graphene Oxide Enhanced Agar Composites: A Biocompatible Photo-catalyst for Degradation of Organic Dyes Shreyas Dindorkar, Jaymin Mistry, Jayesh Hire, Khushi Jain, Nandini Khona, Shreya Peddakolmi, \& Paresh More

The Evolution of Multidrug Resistance in an Isolated Pseudomonas Strain

Allison Grodnick, Ashley Fink, Timothy J. Johnson, \& David Mitchell

Stripping Material from a Supported Lipid Bilayer with High Speed Buffer Flow

Michael J. Ornstead, Ruth Hunter, Mason L. Valentine, Cameron Cooper, Stephen K. Smith, \& Christopher F. Monson

Assessing Initiatives for Rural Health Practices in South Carolina Aalia Soherwardy \& Elizabeth Crouch

Travel Through Time: From 9/11 to COVID-19, Parallel Predictive Analysis of Travel Marketing

Hannah Gilliam 
American Journal of Undergraduate Research (AJUR) is a national, independent, peer-reviewed, open-source, quarterly, multidisciplinary student research journal. Each manuscript of AJUR receives a DOI number. AJUR is archived by the US Library of Congress. AJUR was established in 2002, incorporated as a cheritable not-for-profit organization in 2018. AJUR is indexed internationally by EBSCO and Crossref with ISSNs of 1536-4585 (print) and 2375-8732 (web).

EDITORIAL TEAM Volume 17/ Issue 3 / December 2020

Dr. Kestutis G. Bendinskas, Executive Editor, editor@ajuronline.org

Dr. Anthony Contento, Copy Editor, Treasurer

Peter Newell, Editor, Secretary

Daniel Laird, Web Master

EDITORIAL BOARD by subject area

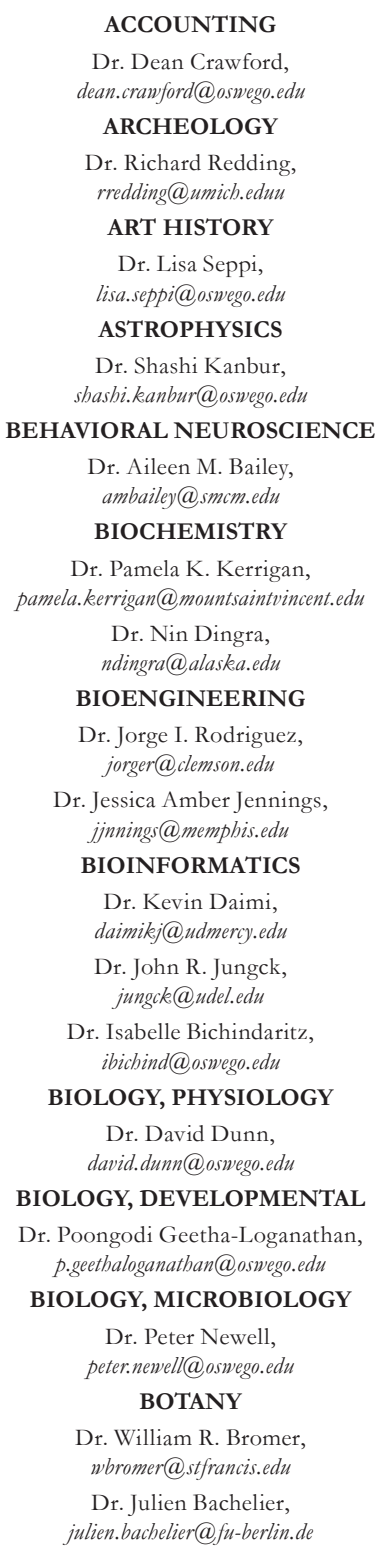

\section{CHEMISTRY}

Dr. Alfredo Castro,

castroa@felician.edu

Dr. Charles Kriley,

cekriley@gcc.edu

Dr. Vadoud Niri,

vadoud.niri@oswego.edu

COMMUNICATION DISORDERS AND SCIENCES

Dr. Kim Tillery,

Kim.Tillery@fredonia.edu

COMPUTER SCIENCES

Dr. Dele Oluwade,

deleoluwade@yaboo.com

Dr. Kevin Daimi,

daimikj@udmercy.edu

Dr. Levent Ertaul,

levent.ertaul@csueastbay.edu

Dr. Mais W Nijim,

Mais.Nijim@tamuk.edu

COMPUTATIONAL CHEMISTRY

Dr. Alexander Soudackov

alexander.soudackov@yale.edu ECOLOGY

Dr. William R. Bromer,

wbromer@stfrancis.edu

ECONOMICS

Dr. Elizabeth Schmitt,

elizabeth.schmitt@oswego.edu

EDUCATION

Dr. Marcia Burrell,

marcia.burrell@oswego.edu

EDUCATION, PHYSICS

Dr. Andrew D. Gavrin,

agavrin@iupui.edu

ENGINEERING, ELECTRICAL

Dr. Michael Omidiora,

michael.omidiora@nyu.edu

ENGINEERING, ENVIRONMENTAL

Dr. Eileen M. Cashman,

eileen.cashman@bumboldt.edu

ENGINEERING, SOFTWARE

Dr. Kevin Daimi, daimik.j@udmercy.edu

\author{
METEOROLOGY \\ Dr. Steven Skubis, \\ steven.skubis@oswego.edu \\ MUSIC \\ Dr. Juliet Forshaw, \\ juliet.forshaw@oswego.edu \\ NANOSCIENCE AND CHEMISTRY \\ Dr. Gary Baker, \\ bakergar@missouri.edu \\ NEUROSCIENCE \\ Dr. Pamela E. Scott-Johnson, \\ pscottj@calstatela.edu \\ PHYSICS \\ Dr. Mohammad Islam, \\ mohammad.islam@oswego.edu \\ Dr. Priyanka Rupasinghe, \\ priyanka.rupasingbe@oswego.edu \\ POLITICAL SCIENCE \\ Dr. Katia Levintova, \\ levintoe@ungb.edu \\ PSYCHOLOGY \\ Dr. Joseph DW Stephens, \\ jdstephe@ncat.edu \\ Dr. Melanie Dyan Hetzel-Riggin, \\ mdh33@psu.edu \\ Dr. Pamela E. Scott-Johnson, \\ pscottj@calstatela.edu \\ SOCIAL SCIENCES \\ Dr. Rena Zito, \\ rzito@elon.edu \\ STATISTICS \\ Dr. Mark Ecker, \\ mark.ecker@uni.edu \\ Dr. Mark Baker, \\ mark.baker@oswego.edu
}

TECHNOLOGY, ENGINEERING

$$
\text { Dr. Reg Pecen, }
$$
regpecen@shsu.edu ZOOLOGY

Dr. Maria Sagot, maria.sagot@oswego.edu 


\title{
Investigating the Effect of Flock Size on Vigilance in the American Coot (Fulica americana) in Relationship to Habitat
}

\author{
Dat Q. Lam, Suyash P. Rizal, Roxanne Cota, Miguel Sicaja, Gabriel Cox, Brandon Wakefield, \& Zia Nisani \\ Department of Biological and Environmental Sciences, Antelope Valley College, Lancaster, CA
}

bttps://doi.org/10.33697/ajur.2020.022

Student:dlam1@avc.edu*,srizal@avc.edu,rcota3@avc.edu,msicaja1@avc.edu,gcox7@avc.edu,bwakefield@avc.edu Mentor:znisani@avc.edu*

\begin{abstract}
Among many anti-predator behaviors, vigilance is observed in many species and plays an important role in survival. In this study, we investigated the effect of flock size on vigilance in American Coots (Fulica americana) foraging on land and water, by observing individual birds in these habitats and recording the time spent scanning (i.e., vigilance). Mean flock size was larger on land compared to water and vigilance negatively correlated with flock size. Birds in water were more vigilant compared to on land, regardless of whether they were foraging alone or in flocks. However, the effect of flock size on vigilance showed a weak linear correlation as it was possible that other factors (e.g., human habituation, food kleptoparasitism, or scramble competition) could have also played a role in shaping vigilance. These results suggest that there is a relationship between flock size and vigilance, which are related to previous researches that show a negative correlation between vigilance and flock size.
\end{abstract}

\section{KEYWORDS}

Birds; American Coot; Vigilance; Scanning; Foraging; Flock Size; Habituation; Competition; Behavior

\section{INTRODUCTION}

Survival of any organism is essential toward reproduction and the passing of genes to the next generation. In nature, perceived predation risk is a major selective force shaping animal behavior. ${ }^{1-3}$ If an animal perceives there is a predator around, it may alter its behavior by decreasing activity level or increasing vigilance. ${ }^{3,4}$

To scan their environment, birds frequently interrupt feeding and lift their heads. ${ }^{5-7}$ This behavior is called vigilance. It has been suggested that there is a negative relationship between vigilance and group size among gregarious species. ${ }^{8,9} \mathrm{~A}$ few literature reviews in birds and mammals have documented a group-size effect on vigilance, providing overwhelming support for theoretical predictions. ${ }^{1,3,10}$

The influence of group size on vigilance has been intensively studied and three hypotheses have been developed to explain why vigilance levels decline with increasing group size. According to the "many eyes" hypothesis, an increase in group size allows for more efficient surveillance, allowing the group to better detect predators while reducing each individual's vigilance.7, 11, 12 A larger group size also enjoys the benefits of group dilution by lowering an individual's risk of predation, as per the "dilution effect" hypothesis. ${ }^{13}$ The decline in individual vigilance can also be supported by the "scramble competition" hypothesis, which explains that the scarcity of resources promotes intraspecific competition, thus lowering each individual's vigilance. $6,12,14,15$

Apart from the effects of group size, other factors also affect vigilance levels. Studies have shown that the characteristics of immediate habitat can also influence vigilance. ${ }^{16-18}$ For example, tall vegetation can serve as a good shelter or refuge by lowering detection and decreasing vulnerability to predators, causing animals to lower their vigilance effort. ${ }^{17}, 19,20$ In American Coot, it has been suggested that birds in water probably experience more safety and as such, we hypothesize that birds foraging on water will be less vigilant that birds on land. ${ }^{21}$

We examined vigilance in the American Coots (Fulica americana), a highly gregarious species found across North America. The species is known to forages both on land and in water, where their diet consists mainly of aquatic and terrestrial vegetation and insects. The objectives of this study were: (1) to investigate the effect of flock size effect on vigilance, and (2) to compare how the effect varies between terrestrial and aquatic habitats. We hypothesized that vigilance would decrease with flock size (due to the many eyes, dilution, and scramble competition effects), and that birds foraging on land would be significantly more vigilant than in water, as they face a broader array of predators. 


\section{METHODS AND PROCEDURES}

Study site

The study was conducted between early September and mid-October, 2019 in Apollo Community Regional Park, Lancaster, CA, before fall migration. The park contains three interconnected, human-made lakes with pavement across the outer edge of the lakes. Along with American Coots, the park is also occupied by other avian species such as Canada Goose (Branta canadensis), Bluewinged Teal (Spatula discors), Eurasian Collared Dove (Streptopelia decaocto), and Great Horned Owl (Bubo virginianus). Being a recreational park, human presence was high, most prominently around noon. The observations were conducted between 7:00 a.m. - 10:00 a.m. in order to minimize the effect of human disturbance.

\section{Field work and data collection}

Observations were made on flock sizes of two or more individuals as well as lone foragers. In order to avoid disturbing the subjects, the observations were made from a pavement at least 15-20 meters away from the coots. For the purpose of the study, vigilance was identified as an individual raising its head (scan) and non-vigilance included behaviors such as pecking the water or ground, preening, walking, or diving. Individual birds were observed using the focal sampling method. ${ }^{22}$ Each observation lasted one minute, during which the time spent scanning (vigilance) was recorded with a stopwatch. During a session, one person reported the observation while another person operated a stopwatch, and recorded the data. Each time the bird switched behavior from vigilance to non-vigilance and vice versa, a lap was taken on the watch. After the one-minute observation, the vigilance laps were added up to determine the total time spent vigilant. In order to keep the flock size the same during each session, the observation was ceased (and not used in analysis) when the flock size changed, the bird went out of sight, or was disturbed by the presence of humans. Data was collected separately for coots foraging in water and on land.

\section{Data analysis}

A total of 630 coots were observed with 324 individuals foraging on land and 306 individuals in water. To prevent pseudoreplication, since the observations were conducted as part of a course-based research project by different individuals at different times, 149 data points from each habitat were randomly selected for analysis. Student's t-test was used to determine if mean flock size and mean vigilance time were significantly different between birds on land and water. To analyze the effect of flock size on vigilance, a Pearson's correlation test was performed. Finally, to investigate the effect of habitats (Land vs water) and birds foraging alone or in a flock, on vigilance (dependent variable) ANOVA test was conducted.

\section{RESULTS}

On land, the number of birds in a flock ranged from $2-21$, with $16.1 \%$ foraging alone. In water, the number of birds in a flock ranged from $2-7$, with $19.5 \%$ foraging alone. On average, the flock size on land was significantly larger than in water, and birds spent significantly less time scanning (vigilance) on land compared to water (Table 1). Regardless of the habitat, birds foraging alone spent significantly more time scanning compared to birds foraging in flocks of two or more $(\mathrm{F} 1,294=22.5$, $\mathrm{p}<0.0001$, Figure 1). Furthermore, birds on land scanned significantly less than birds in water (F 1, $249=146.9$, p < 0.001, Figure 1). Birds alone in water scanned the most while birds in a flock on land scanned the least (Figure 1). There was a weak negative linear relationship between flock size and vigilance on land, $(\mathrm{r}=-0.283, \mathrm{p}=0.013$, Figure 2$)$. There was also a weak negative linear relationship between flock size and vigilance in water, but it was not statistically significant $(r=-0.205, \mathrm{p}=0.077$, Figure 3).

\begin{tabular}{|c|c|c|c|c|c|c|c|}
\hline & \multicolumn{2}{|c|}{ Land } & \multicolumn{2}{c|}{ Water } & \multirow{2}{*}{ d.f. } & t & p \\
\cline { 2 - 7 } & Mean & SD & Mean & SD & & & $<0.001$ \\
\hline Flock size & 5.6 & 4.6 & 2.8 & 1.5 & 296 & 6.89 & $<0.001$ \\
\hline Vigilance (sec) & 21.3 & 16.3 & 41 & 12.2 & 296 & 11.83 & $<$ \\
\hline
\end{tabular}

Table 1. Mean flock size and vigilance for American Coots on land and in water along with results of an independent t-test comparing means. 


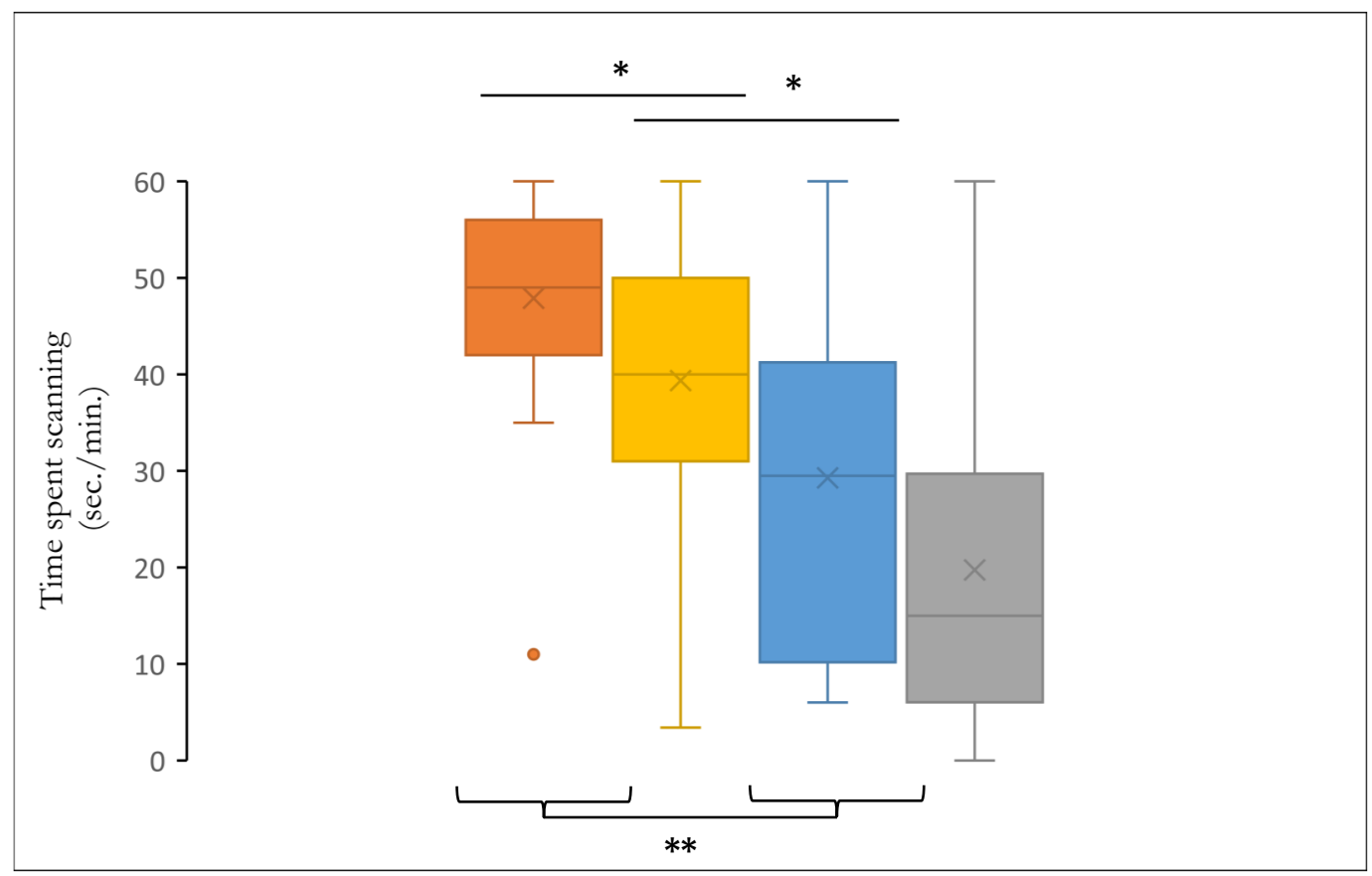

Figure 1. Time birds spent scanning either foraging alone on land ( $\square$ ) or on water $(\square)$, and in a flock on land $(\square)$ or on water $(\square)$. In the box plots, the boundary of the box closest to zero indicates the 25 th percentile, a black line within the box marks the median, and the $\mathrm{X}$ within the box marks the mean. Whiskers above and below the box indicate the 10th and 90th percentiles. Points above and below the whiskers indicate outliers outside the 10th and 90th percentiles. $\left(* \mathrm{p}<0.0001,{ }^{* *} \mathrm{p}<0.001\right)$

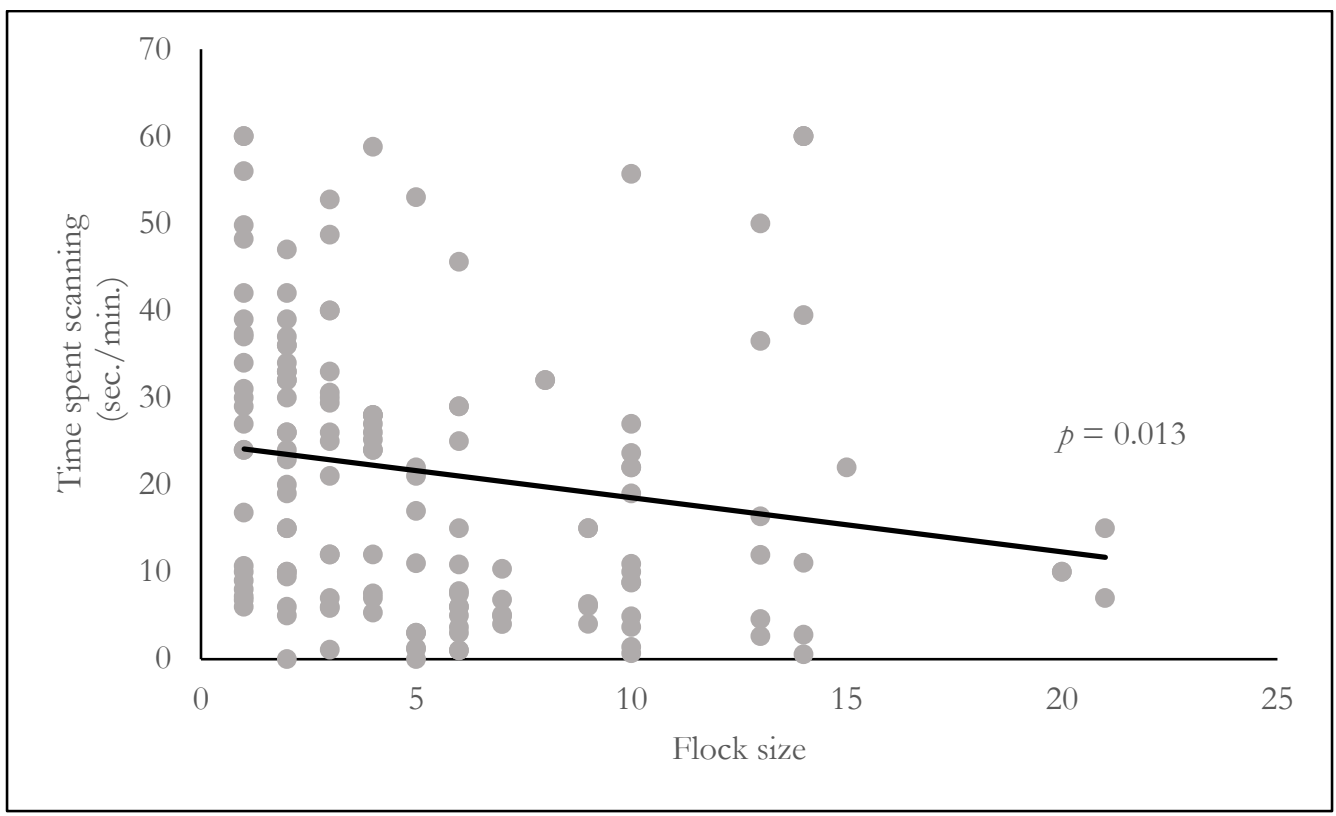

Figure 2. The time spent scanning (vigilant) as a function of flock size of the American Coot on land $(\mathrm{N}=149)$. 


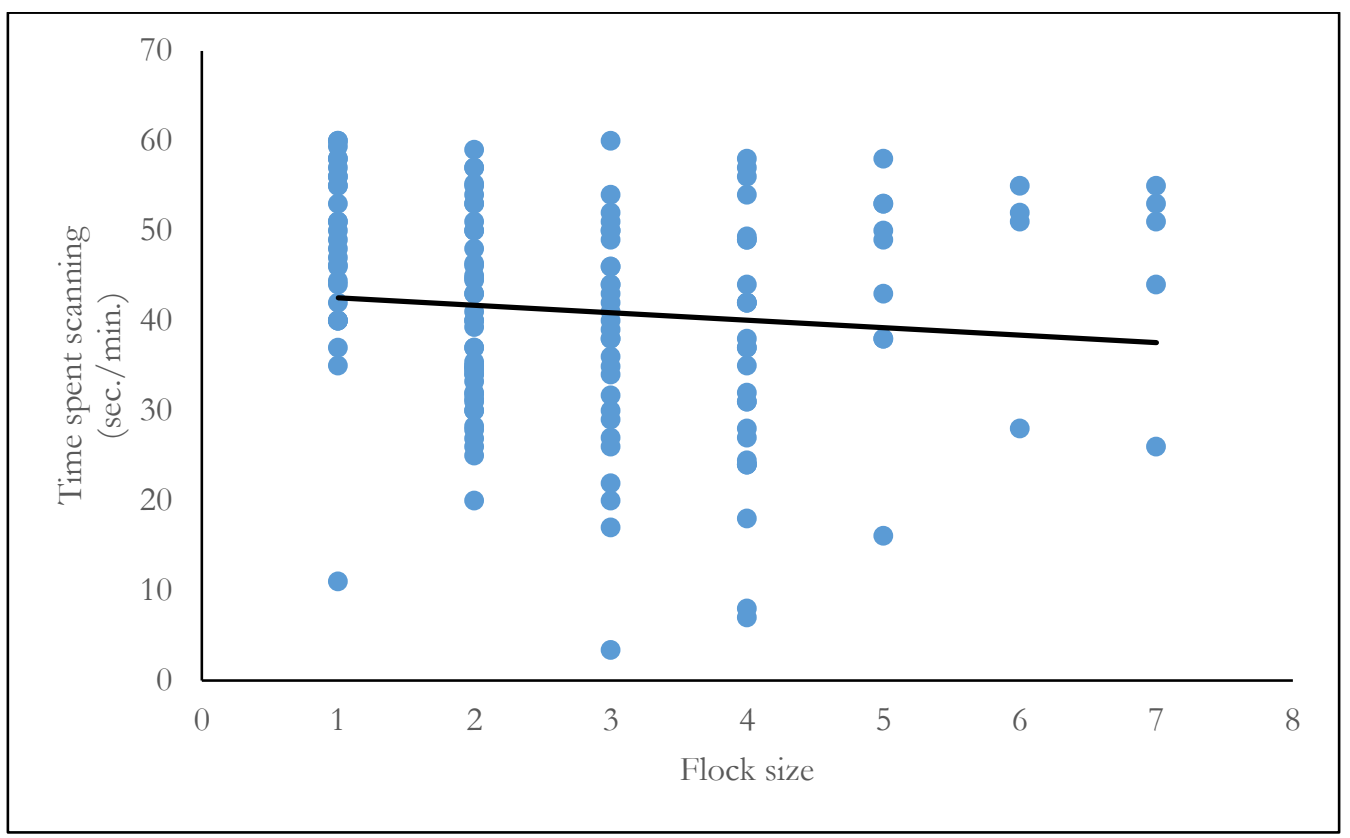

Figure 3. The time spent scanning (vigilant) as a function of flock size of the American Coot in water $(\mathrm{N}=149)$.

\section{DISCUSSION}

The decrease in individual vigilance with increasing group size is one of the most commonly reported relationships in the field of animal behavior. ${ }^{1,10,23}$ Results of this study showed that there was a negative correlation between vigilance and flock size, albeit a weak linear correlation, on land (Figure 2) but not water (Figure 3), coinciding with prior findings for this genus. ${ }^{21,24}$ In addition, the American Coots in this study foraged in larger flocks and were less vigilant while on land compared to water (Table 1).

There are a number of possible explanations to the weak effect of flock size on vigilance in our study. In nature vigilance may serve a number of functions in obtaining information about the environment. ${ }^{25}$ Nevertheless, predator detection is a major function in many species. Thus, one would expect vigilance to increase with the risk of predation and this is supported by many observations of increases in vigilance on or after exposure to a predator. ${ }^{1,10}$ Conversely, reduction or absence of predators would minimize the effect of vigilance. ${ }^{23}$ This might be a reasonable explanation for the weakness of vigilance in the American Coots studied here. Apollo Regional Park is a low-risk habitat for wildlife with little activity of avian predators (Dr. Callyn Yorke of Antelope Valley College, Personal communication) and since the park is fenced off, there are no mammalian predators. While there were park visitors who brought their pets, mostly dogs (Canis familiaris), there were no instances of these animals having predator-prey interactions. Human interactions are often perceived as predatory, which induces fear and physiological changes in animals. This effect has been documented to have significant impact on the foraging efficiency of birds. ${ }^{21,26}$ It is evident that human interactions can cause changes in birds' defense mechanisms, such as vigilance, which directs energy away from foraging in favor of these mechanisms. ${ }^{27}$ As such, human interactions tend to negatively affect reproduction and feeding behaviors of birds, though the degree of impact varies among species. ${ }^{26}$ In certain cases, individuals exposed to prolonged, non-threatening interaction with humans have shown to decrease their vigilance (e.g., acclimation), but still retain a level of 'fear' toward people. ${ }^{26}$ The combination of low predation risk with frequent positive human interaction might have induced habituation to human's presence causing these birds to be relaxed in the presence of humans. Animals that frequently experience nonlethal interactions with humans tend to habituate to humans. ${ }^{28,29}$ This adaptation allows them to optimize their foraging strategy, cutting energywasting escape and vigilance behavior toward non-lethal human disturbance and devoting more time to feeding or preening due to an assessment of low-risk surrounding. In such a situation, factors other than predation might influence vigilance. For example, the opportunity to kleptoparasitize food from conspecifics had the greatest influence on vigilance in Northwestern Crows (Corvus caurinus, recently lumped with American Crow, Corvus brachyrbynchos).$^{30}$ While reduced vigilance for predators in larger groups is a logical hypothesis, the presence of larger groups also may mean more food stealing opportunities and food loss risk for foragers. Coots normally bring their food plants to the water surface to eat them there, and this behaviour allows other waterfowl species to kleptoparasitize them. Herbivorous dabbling ducks, such as American Wigeon (Mareca Americana) and Gadwall (M. strepera), have frequently been observed kleptoparasitizing coots and do reside in Apollo Park. ${ }^{31,32}$ Thus it is reasonable to speculate that kleptoparasitism may have caused the coots in our study to scan more frequently when in water compared to land. 
The presence of more individuals in close proximity, regardless of species, might increase scramble competition, resulting in coots devoting less time to vigilance and more to feeding. ${ }^{33,34}$ In this study, birds foraging alone, regardless of habitat, were significantly more vigilant than birds in flocks (Figure 1). This is to be expected given the greater vulnerability of a single individual to predation and has been extensively documented in other studies. ${ }^{35-37}$ Furthermore, though food density was not measured in the study area, birds on land tended to be in larger flocks compared to water, thus increasing scramble competition. This could have also minimized the strength of the association between vigilance and flock size.

Finally, American Coots either feed in water (by diving, dabbling, or surface feeding) or on land (by grazing or picking up food from the ground). These two environments might be perceived differently in terms of predation risk. Birds on land might be susceptible to both aerial and terrestrial predators, while birds in water are only exposed to aerial threats. In a previous study, feeding-bout lengths in aquatic habitat were longer than terrestrial feeding in Eurasian Coots. ${ }^{21}$ This study suggests that these birds perceived less risk in aquatic habitat and spent more time feeding, thus conversely less time scanning. Though we did not measure feeding bouts, our results contradict (albeit indirectly) these conclusions, as the American coots in this study were more vigilant in water than land whether they were foraging alone or in flocks.

\section{CONCLUSIONS}

We found a significant correlation between flock size and vigilance rate. American Coots were less vigilant in larger flocks and exhibited the highest amount of vigilance when foraging alone. In general, this study is in agreement with previously published studies of this genus. ${ }^{21,24}$ However, many other factors might influence vigilance. ${ }^{10,23}$ We believe that habituation to humans, ${ }^{28,29}$ food kleptoparasitism, ${ }^{30}$ and possibility of scramble competition ${ }^{33,34}$ weakened the relationship between vigilance and flock size. Future studies should investigate individual variation in vigilance of a specific bird, in order to track its patterns of plasticity as the flock size changes.

\section{ACKNOWLEDGMENTS}

This study was conducted as part of a course-based undergraduate research study for Biology 120 (General Organismal, Ecological and Evolutionary Biology) class. We are grateful for all the students that participated in data collection and the anonymous reviewers whose comments/suggestions helped improve this manuscript.

\section{REFERENCES}

1. Lima, S.L., and Dill, L.M. (1990) Behavioral decisions made under the risk of predation: a review and prospectus. Candian Journal of Zoology 68: 619-640. https:// doi.org/10.1139/ 290-092

2. Lima, S.L. (1998) Nonlethal Effects in the Ecology of Predator-Prey Interactions. BioScience 48: 25-34. https://doi.org/10.2307/1313225

3. Caro, T. (2005) Antipredator Defenses in Birds and Mammals. University of Chicago Press, Chicago, IL 592 pages.

4. Kats, L.B., and Dill, L.M. (1998) The scent of death: Chemosensory assessment of predation risk by prey animals. Écoscience vol. 5, no. 3, 1998, p. 361-394. https://doi.org/10.1080/11956860.1998.11682468

5. Bednekoff, P. and Lima, S.L. (2002) Why Are Scanning Patterns So Variable? An Overlooked Question in the Study of AntiPredator Vigilance. Journal of Avian Biology 33(2): 143-149. https:/ / doi.org/10.1034/j.1600-048X.2002.330204.x

6. Beauchamp, G. (2003) Group-size effects on vigilance: a search for mechanisms. Behavioural processes 63: 141-145. https://doi.org/10.1016/S0376-6357(03)00011-1.

7. Randler, C. (2005) Vigilance during Preening in Coots Fulicia atra. Ethology 111: 169_178. https://doi.org/10.1111/j.14390310.2004.01050.x

8. Zhang, Y., Luo, Z., \& Liu, B. (2014) The effect of group size on the vigilance of Mongolian gazelle (Procapra gutturosa). Folia Zoologica 63(2): 81-86. https://doi.org/10.25225/foro.v63.i2.a5.2014

9. Dalerum, F., Lange, H., Skarpe, C., Rooke, T., Inga, B. and Bateman, P. W. (2008) Foraging competition, vigilance and group size in two species of gregarious antelope. South African Journal of Wildlife Research 38(2): 138-145. https:/ / doi.org/ 10.3957/03794369-38.2.138

10. Elgar, M.A. (1989) Predator vigilance and group size in mammals and birds: a critical review of the empirical evidence. Biological Reviews 64: 13-33. https:/ / doi.org/10.1111/j.1469-185X.1989.tb00636.x

11. Pulliam, H.R. (1973). On the advantages of flocking. Journal of theoretical biology 38(2): 419-422. bttps://doi.org/10.1016/0022-5193(73)90184-7.

12. Boukhriss, J., Selmi, S., Béchet, A., and Nouira, S. (2007) Vigilance in Greater Flamingos Wintering in Southern Tunisia: Age-Dependent Flock Size Effect. Ethology 113: 377 - 385. https://doi.org/10.1111/j.1439-0310.2007.01335.x 
13. Hamilton, W.D. (1971) Geometry for the selfish herd. Journal of theoretical biology 31(2): 295-311. bttps:// doi.org/10.1016/0022-5193(71)90189-5

14. Beauchamp, G. (2001) Should vigilance always decrease with group size? Behavioral Ecology and Sociobiology 51: 47-52. https:// doi.org/10.1007/s002650100413

15. Rieucau, G., and Giraldeau, L.A. (2009). Group size effect caused by food competition in nutmeg mannikins (Lonchura punctulata). Behavioral Ecology 20: 421-425. https://doi.org/10.1093/beheco/arn144

16. Metcalfe NB. (1984) The effects of habitat on the vigilance of shorebirds: Is visibility important? Animal Behaviour. 1984;32:981-985. doi: 10.1016/S0003-3472(84)80210-9.

17. Lima SL. (1987) Distance to Cover, Visual obstructions, and vigilance in house sparrows. Behaviour. 1987;102:231-238. doi: 10.1163/156853986X00144.

18. Lazarus J, Symonds M. (1992) Contrasting effects of protective and obstructive cover on avian vigilance. Animal Behaviour. 1992;43:519-521. doi: 10.1016/S0003-3472(05)80110-1.

19. Moreno S, Delibes M, Villafuerte R. (1996) Cover is safe during the day but dangerous at night: the use of vegetation by European wild rabbits. Canadian Journal of Zoology. 1996;74:1656-1660. doi: 10.1139/296-183.

20. Tchabovsky VA, Krasnov B, Khokhlova SI, Shenbrot IG. (2001) The effect of vegetation cover on vigilance and foraging tactics in the fat sand rat Psammomys obesus. Journal of Ethology. 2001;19:105-113. doi: 10.1007/s101640170006.

21. Randler, C. (2006). Feeding Bout Lengths Differ between Terrestrial and Aquatic Feeding Coots Fulica atra. Waterbirds: The International Journal of Waterbird Biology 29(1): 95-99. https:/ / doi.org/ 10.1675/1524-4695(2006)29 [95:FBLDBT]2.0.CO;2

22. Altmann, J. (1974). Observational Study of Behavior: Sampling Methods. Behaviour 49(3/4): 227-267. https:// doi.org/10.1163/156853974X00534

23. Roberts, G. (1996). Why individual vigilance declines as group size increases. Animal Behaviour 51: 1077-1086. https:/ / doi.org/10.1006/anbe.1996.0109

24. Severcan, C.., \& Yamaç, E. (2011). The effects of flock size and human presence on vigilance and feeding behavior in the Eurasian Coot (Fulica atra L.) during breeding season. Acta Ethologica 14(1): 51-56. bttps:// doi.org/10.1007/s10211010-0089-y

25. Lima, S. (1990) Evolutionarily stable antipredator behavior among isolated foragers: On the consequences of successful escape. Journal of Theoretical Biology 143: 77-89. https:/ / doi.org/10.1016/S0022-5193(05)80289-9

26. Price, Megan. (2008) The impact of human disturbance on birds: A selective review, in Too close for comfort (Munn, A., Lunney, D., and Meikle, W., Eds.), 163-196, Royal Zoological Society of New South Wales, Mosmon, Australia. bttps:// doi.org/10.7882/FS.2008.023.

27. Siegel, H. S. (1980). Physiological stress in birds. Bioscience 30: 529-534. https:// doi.org/10.2307/1307973

28. Louis, S. \& Berre, M. (2000). Adjustment of flight distances in Marmota marmota. Canadian Journal of Zoology 78: 556563. https:// doi.org/10.1139/299-242

29. Stankowich, T. (2008). Ungulate flight responses to human disturbance: A review and meta-analysis. Biological Conservation 141: 2159-2173. bttps:/ / doi.org/10.1016/j.biocon.2008.06.026

30. Robinette, R.L., \& Ha, J.C. (2001). Social and ecological factors influencing vigilance by northwestern crows, Corvus caurinus. Animal Behaviour 62: 447-452. bttps:// doi.org/10.1006/anbe.2001.1772

31. Amat, J., \& Soriguer, R. (1984). Kleptoparasitism of Coots by Gadwalls. Ornis Scandinavica (Scandinavian Journal of Ornithology), 15(3), 188-194. doi:10.2307/3675962

32. Eddleman, W.R., Patterson C.T., \& Knopf, F.L. (1985) Interspecific relationships between American coots and waterfowl during fall migration. The Wilson Bulletin, 97(4), 463-472

33. Beauchamp, G. (2007). Competition in foraging flocks of migrating semipalmated sandpipers. Oecologia 154: 403-409. https:// doi.org/10.1007/s00442-007-0818-8

34. Beauchamp, G. (2012). Foraging speed in staging flocks of semipalmated sandpipers: evidence for scramble competition. Oecologia 169(4): 975-980. https:/ / doi.org/10.1007/s00442-012-2269-0

35. Ortiz, C. A., Pendleton, E. L., Newcomb, K. L., \& Smith, J. E. (2019). Conspecific presence and microhabitat features influence foraging decisions across ontogeny in a facultatively social mammal. Behavioral Ecology \& Sociobiology, 73(4), N.PAG. bttps:/ / doi.org/10.1007/s00265-019-2651-6

36. Ridley, A. R., Raihani, N. J., \& Nelson-Flower, M. J. (2008). The cost of being alone: the fate of floaters in a population of cooperatively breeding pied babblers Turdoides bicolor. Journal of Avian Biology, 39(4), 389-392. https:// doi.org/10.1111/j.09088857.2008.04479.x

37. Pettorelli, N., Coulson, T., Durant, S.M., \& Gaillard, J.M. (2011) Predation, individual variability and vertebrate population dynamics. Oecologia 167, 167(2):305-14. https:// doi.org/10.1007/s00442-011-2069-y 


\begin{abstract}
ABOUT STUDENT AUTHORS
At the time of writing, Dat Q. Lam is an undergraduate student at Antelope Valley College, and is transferring to the University of California, Irvine during fall 2020 where he will undertake a B.S in Chemistry. He intends to pursue an M.D in the future with interest in Radiology.
\end{abstract}

Suyash P. Rizal is an undergraduate student at Antelope Valley College, and is transferring to the University of California, Los Angeles in September 2020. He is working towards a B.S. in Molecular, Cell, \& Development Biology and minoring in Biomedical Research. He plans to pursue a medical degree and aims to become a Board-Certified Physician.

Roxanne Cota partook in this research as a 3rd year community college student at Antelope Valley College. She now attends UC Riverside and is pursuing a B.S. in Biological Sciences. Her future education plans include completing a physician assistant master's degree program.

Miguel Sicaja is an undergraduate seeking to obtain a major in Marine Biology. He completed half of his education at Antelope Valley College and will attend UC Santa Barbara for Fall 2020. His major at UC Santa Barbara is Aquatic Biology, in which he hopes to complete his undergraduate education and gain experience in working with aquatic life. After obtaining a bachelor's degree, he will aim to gain a master's degree to further his goals for shark conservation.

Gabriel Cox completed this manuscript as a sophomore at Antelope Valley College, Lancaster, California. He will graduate with an Associate of Science degree in biology and chemistry in the spring of 2021. His future plans are to obtain a graduate degree in molecular biology.

Brandon Wakefield was a sophomore at Antelope Valley College when this research paper was completed. He is currently transferring to University of California, San Diego and will earn a B.A. in Microbiology in the spring of 2022. During his enrollment in UCSD, he plans on exploring the range of occupational opportunities in the field of Microbiology.

\title{
PRESS SUMMARY
}

Many studies about animal's vigilance have shown a negative correlation between flock size and scanning rate among gregarious species. This study investigated the effect of flock size on vigilance in Fulica americana foraging on land and water, adding to the extensive data of anti-predatory behavior among animals. Data was collected by recording the coots scanning rate within their respective habitats. The results show that birds in water were more vigilant compared to the ones on land regardless if they were foraging alone or in flocks. While Fulica Americana exhibits a negative correlation between flock size and vigilance for both habitats, the effect of flock size on vigilance was minimal as it was possible that other factors (human habituation, food kleptoparasitism, or scramble competition) could have also played a role in shaping vigilance. 


\title{
Cyclophosphamide Depletes Ovarian Follicles in Mice During Both the Light and Dark Phases of the Circadian Cycle
}

\author{
Benjamin Z. Koch \& Kristen A. Roosa* \\ Biology Department, State University of New York College at Oneonta, Oneonta, NY \\ https://doi.org/10.33697/ ajur.2020.023 \\ Student: bkoch4@jh.edu \\ Mentor: Kristen.roosa@oneonta.edu*
}

\begin{abstract}
The alkylating agent cyclophosphamide $(\mathrm{CY})$ is a potent ovarian toxicant. It damages growing follicles and causes premature activation and depletion of the resting follicles that constitute the ovarian reserve. While there is abundant information on the impact of CY on the ovary and its toxicity mechanisms, the influence of the circadian rhythm on ovarian toxicity has not been evaluated. To test the hypothesis that time of exposure affects ovarian toxicity of CY, C57BL/6 mice were treated with a single injection of CY $(75 \mathrm{mg} / \mathrm{kg}$ ) at either two hours after lights on (Zeitgeber time (ZT) 02) or two hours after lights off (ZT14). Toxicity was evaluated one week after treatment by counting ovarian follicles in histological sections. Fewer primordial follicles were counted in the ovaries of $\mathrm{CY}$-treated animals at both treatment times, and fewer antral follicles were counted in the ovaries of animals treated at ZT02. There was no difference in the number of primordial follicles in the ovaries of CY-treated animals between the two treatment times. These results demonstrate that CY-induced depletion of the ovarian reserve occurs when mice are exposed early in the light phase and early in the circadian cycle's dark phase. There is no impact of the circadian rhythm on follicle depletion by CY at these time points.
\end{abstract}

\section{KEYWORDS}

Cyclophosphamide; ovary; circadian; ovarian follicles; toxicity; mouse; chronotherapy; alkylating agent

\section{INTRODUCTION}

Female mammals are endowed with a finite number of germ cells (i.e., oocytes) assembled in follicles composed of somatic cells. Non-growing primordial follicles represent a female's ovarian reserve. ${ }^{1,2}$ In healthy females, the number and quality of ovarian follicles decrease gradually throughout life. ${ }^{3}$ Eventually, a high degree of germ cell depletion contributes to ageassociated reproductive senescence and infertility. ${ }^{4,5}$ The age-associated decline in the oocyte reserve may be accelerated by exposure to environmental toxicants that damage ovarian follicles. ${ }^{6-9}$

Many routinely used chemotherapeutic agents are toxic to the ovary. They are a significant source of ovarian toxicant exposure in pre-menopausal women. ${ }^{8,10}$ Alkylating agents are particularly harmful to the ovary. These compounds add alkyl groups to DNA, interfering with transcription, DNA replication, and cell division. ${ }^{11}$ Alkylating agent exposure is associated with a significantly reduced ovarian follicle reserve, ${ }^{12,13}$ amenorrhea, ${ }^{14}$ and premature ovarian insufficiency in women. ${ }^{15}, 16$

Cyclophosphamide (CY) is a bifunctional alkylating agent that induces DNA crosslinks and double-strand breaks. ${ }^{11}$ It is the cornerstone of chemotherapy regimens used to treat cancers in women, such as breast cancer, ovarian cancer, and nonHodgkin lymphoma. ${ }^{17}$ It is also used to treat autoimmune disorders such as systemic lupus erythematosus, ${ }^{18}$ which predominantly affect females. ${ }^{19}$

Cyclophosphamide is a potent ovarian toxicant. Exposure is associated with reduced primordial follicle reserve ${ }^{20}$ and an increased risk of acute ovarian failure in women treated as young adults. ${ }^{14,15}$ In rodents, a single dose $(75 \mathrm{mg} / \mathrm{kg})$ is sufficient to reduce the primordial follicle reserve by $50 \% .^{21,22}$ Cyclophosphamide significantly reduced the number of primordial follicles in human ovarian tissue mouse xenograft experiments. ${ }^{23}, 24$ Treatment of human ovarian xenografts was also associated with increased follicular apoptosis as measured by TUNEL staining ${ }^{24}$ and cleaved caspase-3 quantification. ${ }^{23}$

As cancer survival rates continue to improve, and women delay childbearing to later in life, preserving females' fertility exposed to ovarian toxicants such as $\mathrm{CY}$ has become an important research area. ${ }^{25}$ Current strategies for fertility preservation during chemotherapy include cryopreservation of ovarian tissue, germ cells, or embryos. ${ }^{26}$ Researchers continue to search for less invasive methods to spare females' fertility after exposure to alkylating agents.

The science of chronotherapy involves treating illnesses according to a patient's biological rhythms. The goals of chronotherapy are to minimize toxicity and enhance the effectiveness of pharmaceutical agents. ${ }^{27}$ Twenty-four-hour circadian rhythms in drug response have been observed for many compounds, including at least 40 anticancer agents and ten alkylating 
agents. ${ }^{28}$ Therefore, biological rhythms may be an important consideration during chemotherapy when pre-menopausal women are exposed to alkylating agents.

There is specific evidence that the degree of CY toxicity is influenced by the time of exposure. Male mice treated with CY (3 doses of $150 \mathrm{mg} / \mathrm{kg}$ ) at the light to dark transition of their daily cycle (Zeitgeber time (ZT)14) had better survival (80\%) and retained more bodyweight after CY than those treated at the dark to light transition (ZT02, 20\% ${ }^{29}$. Others had reported the most significant survival rate in mice when they were treated with a single dose of CY $(375 \mathrm{mg} / \mathrm{kg})$ in the four hours around the light to dark transition compared to other times of the day. ${ }^{30}$

While there is abundant information available on the effects of CY on the ovary and its toxicity mechanisms, the influence of the circadian rhythm on its ovarian toxicity has not been evaluated. This study aimed to determine if a circadian rhythm in CY toxicity is evident at a sub-lethal dose that induces significant follicle depletion. A rhythm in ovarian toxicity could be important for CY treatment in women when ovarian follicles should be spared. Furthermore, the circadian rhythm may be an important variable to consider as studies on other ovarian toxicants are planned and reported. We predicted that CY would deplete the ovarian reserve when mice were exposed at both ZT02 and ZT14, but that toxicity would be less severe in the group treated at ZT14. To test this hypothesis, mice were treated with a single dose of CY (75 mg/ $\mathrm{kg})$ at times predicted to yield the most (ZT02) and least (ZT14) overall toxicity. ${ }^{29}$ Ovarian follicles were classified and counted in histological sections one week after treatment.

\section{METHODS AND PROCEDURES}

Animals

Breeding pairs of C57BL/6 mice were obtained from the Jackson Laboratory to generate experimental females for this study. Experimental females were weaned at 21 days old and individually housed in polycarbonate cages with aspen shaving bedding. The light cycle was the main time indicator (Zeitgeber) for the mice in this experiment. All animals were held on a 12-hour light/12-hour dark cycle. The time of lights on was Zeitgeber time 00 (7:00 am EST). Animals were provided with water and chow (Mazuri Rat and Mouse Diet) ad libitum. The temperature was $20 \pm 2{ }^{\circ} \mathrm{C}$ and humidity $30 \pm 10 \%$. All animal procedures were approved by the Institutional Animal Care and Use Committee at the State University of New York at Oneonta (protocol 2016-20).

Treatments

At six weeks old, each female was assigned to one of four treatment groups (seven to eight animals per group). The CYtreated females received an intraperitoneal injection of CY $(75 \mathrm{mg} / \mathrm{kg}$ in saline, Sigma Aldrich) at either ZT02 or ZT14. Control animals received an injection of saline at an equivalent volume at ZT02 or ZT14. All animals were killed at ZT12 by $\mathrm{CO}_{2}$ inhalation one week following the injection. The final body mass of each animal was recorded. The ovaries were removed, dissected free of fat and connective tissue, and weighed. The left ovary was used for histological analysis.

Tissue preparation and histological evaluation

The left ovary from each animal was fixed in 10\% buffered formalin overnight and then serially dehydrated in ethanol. Ovaries were embedded in paraffin and serially sectioned at five $\mu \mathrm{m}$. Every tenth section was placed on a glass slide and stained with hematoxylin and eosin. Sections were viewed under 400X magnification, and ovarian follicles were classified and counted. Oocytes surrounded by a single layer of flattened granulosa cells were classified as primordial. Primary follicles were characterized by an oocyte surrounded by a single layer of cuboidal granulosa cells. The oocytes of secondary follicles were surrounded by more than one layer of cuboidal granulosa cells, and antral follicles were characterized by having multiple layers of cuboidal granulosa cells and an antral space. ${ }^{31}$ Follicles were classified as atretic if the following were observed: degenerating oocyte and/or $>10 \%$ granulosa cells containing apoptotic bodies. ${ }^{32}$ Only follicles containing oocytes with a visible nucleus were counted.

\section{Statistical analysis}

Data were analyzed with JMP version 14.0.0 (SAS Institute, Inc.). A generalized linear model with planned comparisons was used to compare the numbers of follicles (primordial, primary, and secondary, antral, atretic, and total) counted in the ovaries of each CY treated group to controls and among the two treatment times. Levene's test was used to test for equal variance in follicle numbers between females treated at ZT02 and ZT14. All results were expressed as mean \pm standard error of the mean (SEM). The level of significance $(\alpha)$ for each test was 0.05 .

\section{RESULTS}

Representative histological sections from each treatment group are shown in Figure 1. A single dose of cyclophosphamide $(75 \mathrm{mg} / \mathrm{kg}$ ) significantly reduced the number of primordial follicles counted in the ovaries of mice treated both at two hours after lights on (ZT02, 37\% reduction) and two hours after lights off (ZT14, 39\% reduction, Figure 2). However, there was no difference in the number of primordial follicles in the ovaries of females treated at ZT02 and ZT14 (p $=0.64)$, indicating that time of treatment did not affect this measure of ovarian toxicity. In addition to depleting primordial follicles, 
cyclophosphamide reduced the number of antral follicles in the ovaries of mice treated at ZT02. This was not observed in females treated at ZT14, and animals treated at ZT14 had significantly more antral follicles than those treated at ZT02.
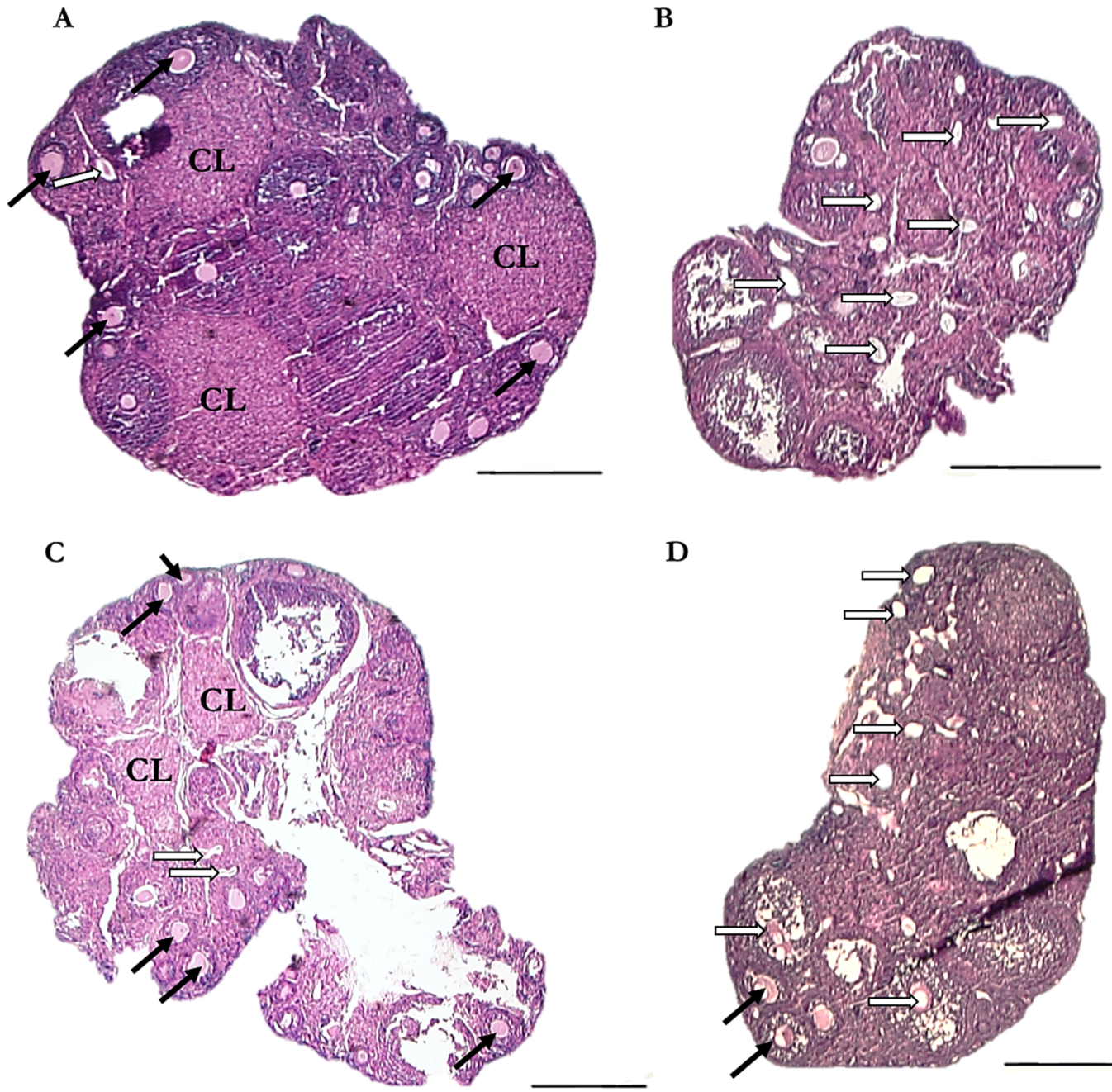

D

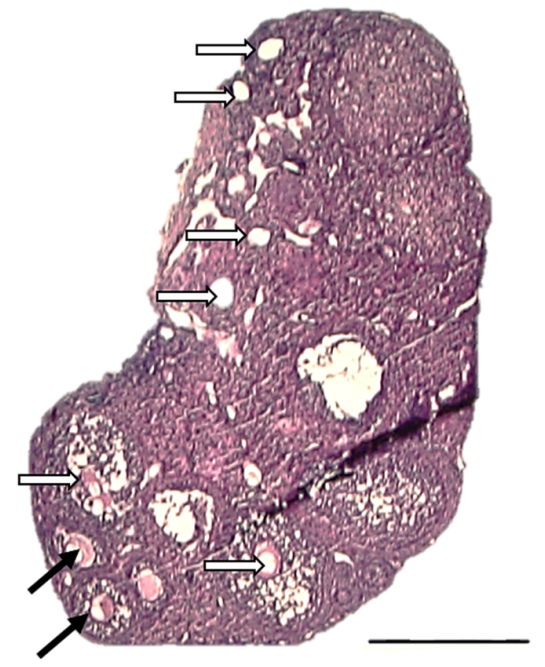

Figure 1. Representative photomicrographs (40X magnification) of ovaries from six-week-old mice treated with saline vehicle at ZT02 (A) or ZT14 (C) or CY at ZT02 (B) or ZT14 (D). Solid arrows indicate healthy secondary follicles, and open arrows indicate remnants of atretic follicles. CL indicates corpora lutea. Scale bars $=500 \mu \mathrm{m}$.

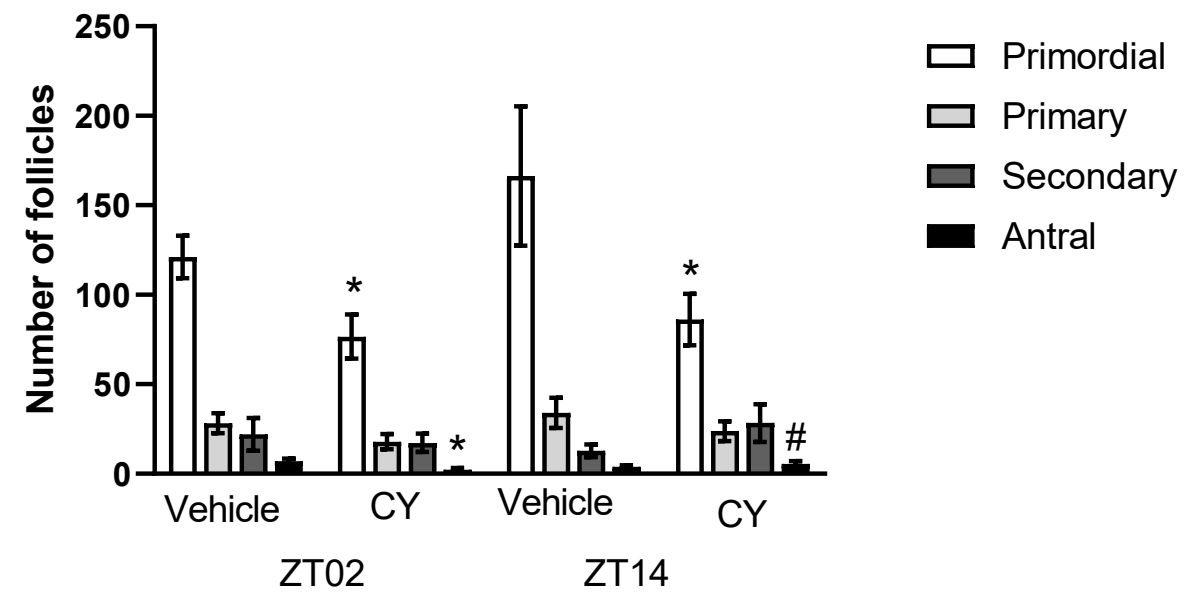

Figure 2. Mean ( \pm SEM) number of follicles counted in every $10^{\text {th }}$ section of one ovary from six-week-old mice treated with $C Y$ or vehicle at ZT02 and ZT14. * indicates a significant difference $(\mathrm{p}<0.05)$ between CY and vehicle-treated animals within the same treatment time, and \# indicates a significant difference between CY-treated animals between ZT02 and ZT14 $(\mathrm{p}=0.034)$. Sample sizes were 4-8 females per group. 
The total number of follicles counted was also compared among the groups. There was a trend toward a decreased total number of follicles counted in the CY group treated at ZT02 as compared to the time-matched controls $(p=0.075$, Figure 3). There was no significant decrease in the number of follicles counted when mice were treated at ZT14 ( $p=0.22)$, and there was no significant difference between treatment times $(\mathrm{p}=0.36)$. Atretic follicles were counted, and there were no differences in the number across any of the comparisons made.

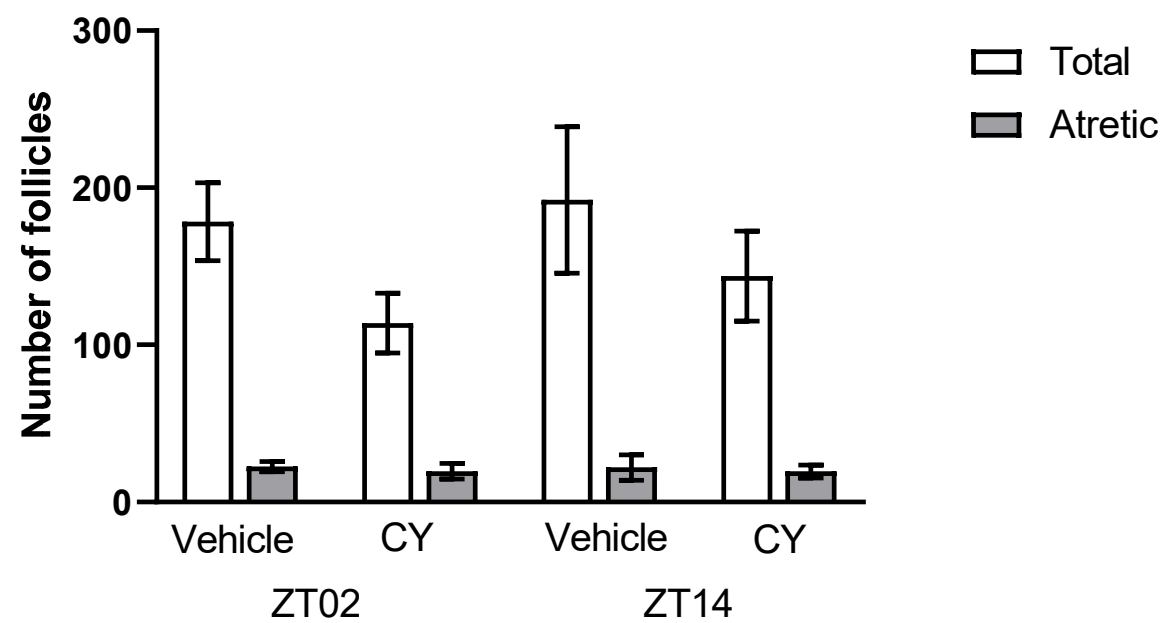

Figure 3. Mean ( \pm SEM) number of follicles of all stages of development combined and atretic follicles counted in every $10^{\text {th }}$ section of one ovary of sixweek-old mice treated with CY or vehicle at ZT02 and ZT14. Sample sizes were 4-8 females per group.

\section{DISCUSSION}

Significant depletion of primordial follicles after CY treatment was expected, based on the results of previous studies using this dose ${ }^{21-33}$. We predicted that females treated at ZT14 would have a greater resistance to CY than those treated at ZT02 (i.e., more primordial follicles remaining after treatment). This prediction was based on the reduced mortality in mice after high-dose CY treatment at ZT14 as compared to ZT02. ${ }^{29,} 30$ The data did not support our hypothesis and suggest that CY reduces the pool of ovarian follicles when animals are exposed both in the light and dark phases of the circadian cycle.

Cyclophosphamide induces premature activation of dormant primordial follicles, which accelerates the depletion of the follicle reserve. ${ }^{33}$ It also induces apoptosis in both dormant and growing follicles. ${ }^{23,24,} 34$ In previous studies, there was at least a $50 \%$ reduction in primordial follicles in mice seven days after a single dose of $75 \mathrm{mg} / \mathrm{kg}$ CY. ${ }^{21,33}$ The degree of follicle depletion by CY in our study is more consistent with the results of Plowchalk \& Mattison, ${ }^{9}$ where there was about a $33 \%$ reduction in primordial follicles.

Others have reported a reduction of antral follicles after CY treatment with either a higher dose $\left(200 \mathrm{mg} / \mathrm{kg}^{9}\right)$ or $\mathrm{more}$ acutely after treatment ( 3 days following an injection of $75 \mathrm{mg} / \mathrm{kg}^{22}$ ). Given that the resting follicle reserve is considered the most representative indicator of long-term ovarian health and fertility, their number is likely more biologically meaningful than the number of antral follicles.

While the atretic follicle number has increased after CY treatment in rodents, it was observed either after in vitro exposure or when ovaries were observed 24 hours after treatment. ${ }^{34,35}$ There were no signs of systemic toxicity after treatment with cyclophosphamide at either time. The change in body mass throughout the treatment or ovary mass did not differ from controls or between CY treatment groups (data not shown).

Dosage is likely a major reason for the presence of a circadian rhythm in CY toxicity when mortality is the endpoint but not for ovarian toxicity. We used a dose that results in depletion of follicles but not systemic toxicity. This dosage (a single injection of $75-100 \mathrm{mg} / \mathrm{kg}^{21,22}$ ) is low compared to the lethal doses used in previous studies (3 doses of $150 \mathrm{mg} / \mathrm{kg}$ ) that demonstrated an impact of the circadian rhythm on toxicity and mortality. Additionally, ovarian toxicity and depletion of follicles require a single injection of $\mathrm{CY}$, whereas circadian effects of toxicity were observed after repeated doses $(3 \times 150$ $\mathrm{mg} / \mathrm{kg}$ ). While multiple doses may better mimic what women undergoing cancer treatment are exposed to, this is difficult to recapitulate due to the great amount of variability in chemotherapy regimens. Whether the circadian rhythm impacts ovarian toxicity after multiple doses of CY remains unknown.

We focused on follicle depletion as the toxicity outcome after CY treatment. This is commonly used as a measure of CY toxicity by many investigators in mechanistic studies and investigations of potential ameliorators of ovarian damage. ${ }^{22,23,33,36,}$ 
${ }^{37}$ It is unlikely that the dose we administered would significantly impact fertility one week after treatment, ${ }^{21}$ therefore, it was not tested. However, it remains unknown if the circadian rhythm would impact fertility after CY treatment in the long term or in aged animals with an already diminished primordial follicle reserve.

Given current information on circadian patterns in toxicity in rodents, it seems unlikely that other points in the circadian cycle would yield different results in ovarian toxicity. We evaluated time points based on the maximum and minimum times of sensitivity for a high dose of cyclophosphamide $(300-375 \mathrm{mg} / \mathrm{kg}) \cdot{ }^{29},{ }^{30} \mathrm{In}$ rodents, significant differences in sensitivity to several other toxicants have been observed when animals were exposed just after lights on and just after lights off, ${ }^{38-40}$ including the toxicity of the alkylating agent cisplatin. ${ }^{40}$ Given these trends, it is unlikely that other times in the circadian cycle would impact ovarian toxicity of CY (e.g., middle of dark or light phases).

\section{CONCLUSIONS}

To our knowledge, this is the first evaluation of the potential impact of the circadian rhythm on ovarian toxicity. We report that time of treatment does not impact the toxicity of $\mathrm{CY}$, in terms of follicle depletion, at two specific times in the circadian rhythm. Future studies to evaluate the influence of time of treatment with longer-duration dosing regimens or in combination with other alkylating chemotherapeutic agents may shed more light on the role the circadian rhythm may play in ovarian toxicity of CY.

\section{ACKNOWLEDGEMENTS}

This research was funded by the Research Foundation for the State University of New York. The authors thank Morgan Gray, Brittany Riddick, and Coreena Monroe for their technical assistance and Dr. Jennifer Nagashima for her review of this manuscript. We also thank the anonymous reviewers for their constructive feedback on an earlier draft of this article.

\section{REFERENCES}

1. Gougeon A. (2004) Dynamics of human follicular growth: morphologic, dynamic, and functional aspects, in The Ovary (Leung, P. \& Adashi E., Eds.) 2nd ed., 25-38. Elsevier Academic Press, San Diego, CA.

2. Kerr, J. B., Myers, M., and Anderson, R. A. (2013) The dynamics of the primordial follicle reserve, Reproduction 146(6), R205-R215. bttps:// doi.org/10.1530/ rep-13-0181

3. Faddy, M.J. (2000) Follicle dynamics during ovarian ageing, Mol Cell Endocrinol 163, 43-48. https://doi.org/10.1016/s03037207(99)00238-5

4. Gosden, R. G., Laing, S. C., Felicio, L. S., Nelson, J. F., and Finch, C. E. (1983) Imminent oocyte exhaustion and reduced follicular recruitment mark the transition to acyclicity in aging C57Bl/6J mice, Biol Reprod 28(2), 255-260. bttps:// doi.org/ 10.1095/ biolreprod28.2.255

5. Nelson, J. F., Felicio, L. S., Randall, P. K., Sims, C., and Finch, C.E. (1982) A longitudinal study of estrous cyclicity in aging c57bl-6j mice: cycle frequency, length and vaginal cytology, Biol Reprod 27(2), 327-339. bttps:/ / doi.org/10.1095/biolreprod27.2.327

6. Dyer, C.A., Raymond-Whish, S., Schmuki, S., Fisher, T., Pyzyna, B., Bennett, A., and Mayer, L. P. (2013) Accelerated follicle depletion in vitro and in vivo in Sprague-Dawley rats using the combination of 4-vinylcyclohexene diepoxide and triptolide, J Zoo Wildl Med 44(4 Suppl), S9-17. bttps:/ / doi.org/ 10.1638/1042-7260-44.4s.s9

7. Igawa, Y., Keating, A. F., Rajapaksa, K. S., Sipes, I. G., and Hoyer, P. B. (2009) Evaluation of ovotoxicity induced by 7 , 12-dimethylbenz[a]anthracene and its 3,4-diol metabolite utilizing a rat in vitro ovarian culture system. Toxicol Appl Pharmacol, 234(3), 361-369. https:// doi.org/10.1016/j.taap.2008.10.009

8. Meirow, D., and Nugent, D. (2001) The effects of radiotherapy and chemotherapy on female reproduction. Hum Reprod Update, 7(6), 535-543. bttps:// doi.org/10.1093/ humupd/7.6.535

9. Plowchalk, D. R., and Mattison, D. R. (1992) Reproductive toxicity of cyclophosphamide in the C57BL/6N mouse: 1. Effects on ovarian structure and function, Reprod Toxicol, 6(5), 411-421. https:// doi.org/10.1016/0890-6238(92)90004-d

10. Mark-Kappeler, C. J., Hoyer, P. B., and Devine, P. J. (2011) Xenobiotic effects on ovarian preantral follicles, Biol Reprod 85(5), 871-883. bttps:/ / doi.org/10.1095/ biolreprod.111.091173

11. Fu, D., Calvo, J. A, and Samson, L. D. (2012) Balancing repair and tolerance of DNA damage caused by alkylating agents, Nat Rev Cancer 12(2), 104-120. https:/ / doi.org/10.1038/nrc3185

12. Gracia, C. R., Sammel, M. D., Freeman, E., Prewitt, M., Carlson, C., Ray A., Vance, A., and Ginsberg, J. P (2012) assessing the impact of cancer therapies on ovarian reserve, Fertil Steril 97(1), 134-140. https:// dx.doi.org/10.1016\%2Fj.fertnstert.2011.10.040

13. Oktem, O., and Oktay, K. (2007) Quantitative assessment of the impact of chemotherapy on ovarian follicle reserve and stromal function, Cancer 110(10), 2222-2229. https:/ / doi.org/10.1002/ cncr.23071

14. Minton, S. E., and Munster, P. N. (2002) Chemotherapy-induced amenorrhea and fertility in women undergoing adjuvant treatment for breast cancer, Cancer Control 9(6), 466-472. https:// doi.org/10.1177/107327480200900603

15. Chemaitilly, W., Mertens, A. C, Mitby, P., Whitton, J., Stovall, M., Yutaka, Y., Robinson, L. L., and Sklar, C. A. (2006) Acute ovarian failure in the childhood cancer survivor study, J Clin Endocrinol Metab 91(5), 1723-1728.

bttps:// doi.org/10.1210/jc.2006-0020 
16. Chemaitilly, W., Li, Z., Krasin, M. J., Brooke, R. J., Wilson, C. L., Green, D. M., Klosky, J. L., Barnes, N., Clark K. L., Farr, J. B., Fernandez-Pineda, I., Bishop, M. W., Metzger, M., Pui, C., Kaste, S. C., Ness, K. K., Srivastava, D. K., Robison, L. L., Hudson, M. M., Yasui, Y. and Sklar, C. A. (2017) Premature ovarian insufficiency in childhood cancer survivors: A report from the St. Jude Lifetime Cohort, J Clin Endocrinol Metab 102(7), 2242-2250. https:// doi.org/10.1210/jc.2016-3723

17. Emadi, A., Jones, R. J., and Brodsky, R. A. (2009) Cyclophosphamide and cancer: golden anniversary, Nat Rev Clin Oncol 6(11), 638-647. https:// doi.org/10.1038/nrclinonc.2009.146

18. Lisnevskaia, L., Murphy, G., and Isenberg, D. (2014) Systemic lupus erythematosus, Lancet, 384(9957), $1878-1888$. bttps:/ / doi.org/10.1016/S0140-6736(14)60128-8

19. Cooper, G. S., and Stroehla, B. C. (2003) The epidemiology of autoimmune diseases, Autoimmun Rev 2(3), $119-125$. bttps:// doi.org/10.1016/s1568-9972(03)00006-5

20. Marcello, M. F., Nuciforo, G., Romeo, R., Di Dino, G., Russo, I., Russo, A., Palumbo, G., and Schiliro, G. (1990) Structural and ultrastructural study of the ovary in childhood leukemia after successful treatment, Cancer 66(10), 20992104. bttps:/ / doi.org/ 10.1002/1097-0142(19901115)66:10\%3C2099::AID-CNCR2820661010\%3E3.0.CO;2-3

21. Meirow, D., Lewis, H., Nugent, D., and Epstein, M. (1999) Subclinical depletion of primordial follicular reserve in mice treated with cyclophosphamide: clinical importance and proposed accurate investigative tool, Hum Reprod 14(7), 19031907. https:// doi.org/10.1093/ bumrep/14.7.1903

22. Plowchalk, D. R., and Mattison, D. R. (1991) Phosphoramide mustard is responsible for the ovarian toxicity of cyclophosphamide, Toxicol Appl Pharmacol 107(3),472-481. bttps:/ / doi.org/10.1016/0041-008x(91)90310-b

23. Li, F., Turan, V., Lierman, S., Cuvelier, C., De Sutter, P., and Oktay, K. (2014) Sphingosine-1-phosphate prevents chemotherapy-induced human primordial follicle death, Hum Reprod 29(1), 107-113. bttps://doi.org/10.1093/ bumrep/det391

24. Oktem, O., and Oktay, K. (2007) A novel ovarian xenografting model to characterize the impact of chemotherapy agents on human primordial follicle reserve, Cancer Res 67(21), 10159-10162. https:/ / doi.org/ 10.1158/0008-5472.can-07-2042

25. Roness, H., Kashi, O., and Meirow, D. (2016) Prevention of chemotherapy-induced ovarian damage, Fertil Steril 105(1), 20-29. https:// doi.org/10.1016/j.fertnstert.2015.11.043

26. Kim, S. Y., Kim, S. K,. Lee, J. R., and Woodruff, T. K. (2016) Toward precision medicine for preserving fertility in cancer patients: existing and emerging fertility preservation options for women, J Gynecol Oncol 27(2), e22 bttps://doi.org/10.3802/jgo.2016.27.e22

27. Ballesta, A., Innominato, P., Dallmann, R., Rand, D., and Levi, F. (2017) Systems chrontherapeutics, Pharmacol Rev 69(2), 161-199. https:// doi.org/10.1124/pr.116.013441

28. Lévi, F., Okyar. A., Dulong, S., Innominato, P. F., and Clairambault, J. (2010) Circadian timing in cancer treatments, Anпu Rev Pharmacol Toxicol 50, 377-421. https:// doi.org/10.1146/ annurev.pharmtox.48.113006.094626

29. Gorbacheva, V. Y., Kondratov, R. V., Zhang, R., Cherukuri, S., Gudkov, A. V., Takahashi, J. S, and Antoch, M. P. (2005) Circadian sensitivity to the chemotherapeutic agent cyclophosphamide depends on the functional status of the CLOCK/BMAL1 transactivation complex, Proc Natl Acad Sci USA., 102(9), 3407-3412. https:// doi.org/10.1073/pnas.0409897102

30. Haus, E., Fernandes, G., Kühl, J. F., Yunis, E. J. , Lee, J. K., and Halberg, F. (1974) Murine circadian susceptibility rhythm to cyclophosphamide, Chronobiologia 1(3), 270-277.

31. Place, N. J., and Cruickshank, J. (2009) Graded response to short photoperiod during development and early adulthood in Siberian hamsters and the effects on reproduction as females age, Horm Behav 55(3), 390-397. bttps:// doi.org/10.1016/j.ybbeh.2009.01.005

32. Greenfield, C., Babus, J., Furth, P., Marion, S., Hoyer, P., and Flaws, J. (2007) BAX is involved in regulating follicular growth, but is dispensable for follicle atresia in adult mouse ovaries, Reproduction 133(1), 107-116. https:/ / doi.org/10.1530/ rep-06-0144

33. Kalich-Philosoph L, Roness H, Carmely A, Fishel-Bartal, M., Ligumsky, H., Paglin, S., Wolf, I., Kanety, H., Sredni, B., and Meirow, D. (2013) Cyclophosphamide triggers follicle activation and "burnout"; AS101 prevents follicle loss and preserves fertility, Sci Transl Med. 5(185), 185ra162. https://doi.org/10.1126/scitranslmed.3005402

34. Desmeules, P., and Devine, P.J. (2006) Characterizing the ovotoxicity of cyclophosphamide metabolites on cultured mouse ovaries, Toxicol Sci 90(2), 500-509. https:// doi.org/10.1093/toxsci/kfj086

35. Lopez, S.G, and Luderer, U. (2004) Effects of cyclophosphamide and buthionine sulfoximine on ovarian glutathione and apoptosis, Free Radic Biol Med 36(11), 1366-1377. https:/ / doi.org/10.1016/j.freeradbiomed.2004.02.067

36. Meirow, D, Assad, G., Dor, J., and Rabinovici J. (2004) The GnRH antagonist cetrorelix reduces cyclophosphamideinduced ovarian follicular destruction in mice, Hum Reprod 19(6),1294-1299. https:// doi.org/10.1093/ humrep/deh257

37. Madden, J. A., Hoyer, P. B, Devine, P. J, and Keating, A. F. (2014) Involvement of a volatile metabolite during phosphoramide mustard-induced ovotoxicity, Toxicol Appl Pharmacol 277(1),1-7. bttps:// doi.org/10.1016/j.taap.2014.03.006

38. DeBruyne, J. P, Weaver, D. R, and Dallmann, R. (2014) The hepatic circadian clock modulates xenobiotic metabolism in mice, J Biol Rhythms 29(4), 277-287. https:/ / doi.org/10.1177/0748730414544740

39. Johnson, B. P., Walisser, J. A., Liu, Y., Shen, A. L., McDearmon, E. L., Moran, S. L., McIntosh, B. E., Vollrath, A. L., Schook, A. C., Takahashi, J. S., and Bradfield, C. A. (2014) Hepatocyte circadian clock controls acetaminophen bioactivation through NADPH-cytochrome P450 oxidoreductase, Proc Natl Acad Sci USA. 111(52), 18757-18762. https:// doi.org/10.1073/pnas.1421708111 
40. Dakup, P. P., Porter, K. I., Little, A. A., Gajula, R. P., Zhang, H., Skornyakov, E., Kemp, M. G., Van Dongen, H. P. A., and Gaddameedhi, S. (2018) The circadian clock regulates cisplatin-induced toxicity and tumor regression in melanoma mouse and human models, Oncotarget 9(18), 14524-14538. https:// doi.org/ 10.18632/ oncotarget.24539

\section{ABOUT STUDENT AUTHOR}

Benjamin Koch recently graduated from SUNY Oneonta with a B.S. with a focus in biology and organic chemistry. Benjamin's interest in preserving the health of the environment for the enjoyment and use of future generations was inspired by his time spent researching, and SCUBA diving in, the freshwater lakes and stream surrounding Oneonta and Cooperstown. He is currently working towards his Master's Degree in Environmental Science and Policy at John Hopkins University. He plans to one day be able to enact meaningful environmental solutions that will help mitigate the current effects of global climate change on natural resources.

\section{PRESS SUMMARY}

Many of the chemicals used to treat cancer patients also have negative side effects, including damaging the ovary. cyclophosphamide (CY) is one such chemical commonly used during chemotherapy. CY is a known alkylating agent that interferes with DNA replication cell division. This is useful for fighting cancer cells, but it also damages egg cells (oocytes). Finding ways to mitigate the harmful effects of these ovarian toxicants was a primary focus of this study. One theory that was tested involved giving female mice sub-lethal dosages of the CY at two different stages of their circadian rhythm (in the evening vs. the morning). We hypothesized that the mice who receive the treatment when they are most metabolically active (in the evening) would have a higher resistance to the toxicant. These mice would be presumed to have more viable ovarian follicles left intact after the treatment. While previous studies have shown that chronotherapy is a viable method in reducing mice mortality to a lethal dosage of $\mathrm{CY}$, no prior research has shown how the resistance of ovarian follicle health to toxins can be linked to metabolic rate. Our results suggest that the circadian rhythm has no significant effect on the mice's ovarian resistance to $\mathrm{CY}$ at times tested. 


\title{
Associations of Maternal Controlling Feeding Practices with Child Internalizing Symptoms and Body Mass Index in Ethnically-Diverse Mother-Child Dyads
}

\author{
Paulina Mozdzierz, Genevieve F. Dunton, \& Tyler B. Mason* \\ Department of Preventive Medicine, University of Southern California, Los Angeles, CA \\ bttps:/ / doi.org/10.33697/ ajur.2020.024 \\ Student:pmozdzie@usc.edu \\ Mentors: tylermas@usc.edu*,dunton@usc.edu
}

\begin{abstract}
Mothers may use controlling feeding practices (i.e., pressure to eat and restriction) to regulate their child's weight. However, these practices may have unintended consequences on the weight and mental health of children. The first aim of this study was to investigate differences in maternal controlling feeding practices by child gender, age, and maternal ethnicity. The second aim was to examine cross-sectional associations among maternal controlling feeding practices, child body mass index $z$-scores (BMI-z), global internalizing symptoms (i.e., depression and anxiety symptoms), and self-esteem. The third aim was to determine whether child sex and mother ethnicity moderate these associations. A sample of 202 ethnically diverse mother-child dyads (children ages $8-12 ; 49 \%$ female) completed self-report questionnaires and had weight and height measurements taken. Results showed no differences in maternal controlling feeding practices by gender, ethnicity, or age. Pressure to eat was negatively related to child BMI-z, and restriction was positively related to BMI-z. Moreover, pressure to eat was negatively related to child self-esteem. There were no associations between maternal controlling feeding practices and global internalizing symptoms. Further, no associations differed by child gender or mother ethnicity. Maternal controlling feeding practices may be used to move a child's weight toward a healthy weight range. Overall, there was little evidence for associations between feeding practices and poor mental health; although, pressure to eat was related to poorer self-esteem in children.
\end{abstract}

\section{KEYWORDS}

Maternal; Feeding; Practices; Child; BMI-z; Mental; Health; Controlling; Restricting

\section{INTRODUCTION}

Obesity is increasingly becoming a concern in children of all ages, genders, and ethnicities. ${ }^{1,2}$ Similar to statistics for obesity, approximately one in every five children and adolescents have a mental health problem. ${ }^{3-6}$ Higher body weight and mental health problems, both of which have been found to correlate with controlling feeding practices, can lead to numerous health complications that may persist well into adulthood. ${ }^{5,6,7}$ Further, studies have found associations between higher body weight in childhood and an increased prevalence of anxiety and mood disorders later in life. ${ }^{8}$ Particularly, middle childhood is a time where mental health disorders and weight gain begin developing. ${ }^{6,8,11-13}$

While obesity itself may lead to negative consequences such as depression, anxiety disorders, and chronic disease risk, ${ }^{8,14-16}$ it is possible that efforts used to control children's weight, such as controlling feeding practices, may contribute to these factors and pose an even greater risk for these physical and mental health problems. ${ }^{8}$ Previous studies have found that controlling feeding practices can contribute to a failure to appropriately respond to hunger or fullness signals, which can lead to weight gain. Research suggests that children tend to overeat when given free access to foods that are typically restricted from their diet.17,18 Further, feeding practices may lead to the development of weight and eating behavior concerns and a lack of autonomy. ${ }^{19}$ Additional research in adolescents found that maternal controlling feeding practices were positively correlated with bulimic symptoms (in females) and negatively correlated with self-esteem. ${ }^{6,20-21}$

Given healthcare providers' focus on the importance of proper nutrition and maintenance of a healthy weight in children,,10 parents may use controlling feeding practices (i.e., restricting child's food intake and pressuring a child to eat) in order to help their child achieve and maintain a healthy weight, which is defined by body mass index (BMI) for age and sex from the 5th through the 85th percentile. ${ }^{11}$ Parents may restrict eating by limiting meal portions and commenting on the large intake of food by their child, 
or parents may pressure their child to eat by forcing their child to consume an entire meal or punishing their child for failure to finish a meal. ${ }^{12,20,22-24}$ Among infants and toddlers, controlling feeding practices, both restriction and pressure to eat, have been shown to predict lower weight in children. ${ }^{11-12}$ Despite being predictive of lower weight in infants, restrictive and controlling feeding practices may be associated with poor weight and mental health-related outcomes in older children. ${ }^{8,12-13}$

Research findings have not been consistent regarding the relationship between parental controlling feeding practices and child weight status. Some research has found positive associations between restrictive feeding practices and weight and negative associations between pressure to eat and weight in early childhood.13,25-26 This finding could be due to child overeating behavior when given the opportunity to eat away from the supervision of a restrictive parent. ${ }^{27}$ Pressure to eat may reduce weight due to the development of aversion or anxiety towards food; in the absence of parental pressure, the child refuses to eat. ${ }^{27-28}$ In contrast, other studies of toddlers found no relation between child BMI-z scores and maternal controlling feeding practices; ${ }^{27}$ Similarly, in separate studies of Hispanic preschool children, there was no relation between maternal controlling feeding practices and BMI-z scores. ${ }^{23,29}$

Empirical research has primarily focused on how maternal feeding practices can affect the physical weight of a child. Thus, there is a lack of data on the relationship between maternal feeding practices and child internalizing symptoms and self-esteem. One study found a positive relationship between increased maternal internalizing symptoms and increased controlling feeding practices ${ }^{30}$ but studies have yet to look at child internalizing symptoms. Studies have found positive correlations between restrictive maternal feeding practices and disordered eating, such as binge eating in adolescents. ${ }^{20,24}$ Although disordered eating is highly associated with internalizing symptoms, ${ }^{31}$ it is unclear if the link between maternal controlling feeding practices extends to internalizing symptoms, such as mood and anxiety symptoms.

The Role of Child Gender

Gender may be an important variable to consider when examining the associations between maternal feeding practices and child internalizing symptoms and weight. Findings on gender and maternal feeding practices are varied; some studies suggest that maternal restriction is associated with self-esteem in girls with obesity and also associated with food intake control in girls, yet no significant associations were found for boys. ${ }^{32}$ This finding suggests that girls may be disproportionately affected by controlling feeding practices. Additional research observed that maternal pressure to eat in children 7-12 years old was linked to greater consumption of food in the absence of hunger, and a stronger correlation was observed for boys compared to girls. ${ }^{21.33-34}$ One study found that fathers reported more pressure to eat for boys than girls. ${ }^{35}$ However, in a separate study comparing maternal and paternal feeding practices among parents of boys and girls, there were no differences. ${ }^{20}$ Additional research found that maternal feeding is more likely to occur between mother-daughter than mother-son relationships; one possible explanation for this difference is the societal pressure to adhere to gender-related dietary norms. ${ }^{36-38}$ These associations provide preliminary evidence of gender being a possible variable to consider when assessing maternal controlling feeding practices and their relation to child weight and internalizing symptoms.

\section{The Role of Ethnicity}

In addition to gender, ethnicity may be an important factor to consider. Lifestyle differences between Hispanic and non-Hispanic families may lead to behavior that results in varied child weight and internalizing symptoms. Findings are mixed. However, some evidence suggests that non-White Hispanic mothers restrict foods less than White mothers. Other studies did not find evidence to support this; some research has found that Hispanic mothers were more likely to pressure their children to eat. ${ }^{38-39}$ One study found that non-White parents, including Hispanic parents, engaged in more restrictive and pressuring feeding practices. ${ }^{40}$

Differences between Hispanic and non-Hispanic mothers may be linked to Hispanic culture, which emphasizes social eating. ${ }^{41-43}$ In Hispanic culture, the ideal maternal figure is closely associated with feeding children; the more food, the more nurturing a mother is. ${ }^{44}$ This can lead to maternal pressuring to eat. Research suggests that Hispanic mothers have a different idea of what being overweight is, and thus they participate in pressuring to eat at times when non-Hispanic parents would not. ${ }^{45-46}$ Studies indicate that non-White Hispanic mothers may prefer their children to be heavier. They believe that this indicates child health and strength; this mentality can lead to more pressuring and less restrictive feeding practices. ${ }^{41,45-47}$

\section{The Current Study}

Varying patterns of feeding practices by gender and ethnicity have led us to expect differences between Hispanic and nonHispanic children in relation to how maternal feeding patterns affect child internalizing symptoms and weight. We expect these differences due to cultural differences; for example, Hispanics regard food as a core part of their culture and thus are less likely to restrict it. ${ }^{19}$ Gender differences are expected due to the societal norms placed on females and males, such as that females should eat less than males, as well as the difference in mother-daughter and mother-son relationships. ${ }^{36-37,48}$ These patterns could explain 
differences in child obesity and internalizing symptoms, thus establishing the importance of the further study of these associations. ${ }^{15}$

Studying the effect of maternal controlling feeding practices on adolescents, ages $8-12$, is important because adolescence is a time where children strive to be more independent; they make their own decisions, yet decisions are still influenced by decisions made for them growing up. Additionally, mental health disorders are emerging in this age group, and it is important to study the role of controlling feeding practices in this trend. $6,8,11-13$

The current study had several goals. The first research question examined differences in maternal controlling feeding practices by child gender, age, and maternal ethnicity. Age was examined as children become more independent as they get older, and mothers' controlling feeding practices may change due to increased autonomy. ${ }^{49}$ The second research question was examined to see if bivariate associations were among maternal feeding practices, child BMI, child internalizing symptoms (i.e., depressive and anxiety symptoms), and self-esteem. The third research question examined child gender and maternal ethnicity as moderators of associations between maternal controlling feeding practices and child BMI, internalizing symptoms, and self-esteem. The study aims to add knowledge to a topic that has not been extensively studied. Specifically, findings will allow us to understand the effect of controlling feeding practices on children's mental health and how this may differ for children of different gender and ethnicity. Findings may be used to develop interventions to educate parents on controlling eating habits that best promote healthy child weight and mental health, thus bettering health outcomes during adolescence and into adulthood.

\section{METHODS AND PROCEDURES}

\section{Participants and Procedure}

The current study sample was drawn from Wave one of the Mothers, and Their Children's Health (MATCH) study, which includes 202 dyads of ethnically diverse mothers and their 8-12-year-old children (age range during baseline assessment). The sample included 103 boys and 99 girls, and the mean age of children was 9.60 (SD=0.91). There were 99 mothers (49\% of dyads) who indicated being Hispanic or Latina, with 103 being non-Hispanic. Mother-child dyads were recruited from urban schools in the greater Los Angeles community. Children were in third through sixth grade. Inclusion criteria included $\geq 50 \%$ custodianship of the child with the mother and ability of mothers and children to read in either English or Spanish. The study exclusion criteria for children or mothers: (1) currently taking medications for thyroid function or psychological conditions such as depression, anxiety, mood disorders, and ADHD, (2) health issues that limit physical activity, (3) enrolled in special education programs, (4) currently using oral or inhalant corticosteroids, (5) pregnancy, (6) mother works more than two weekday evenings per week (e.g., between 5 and $9 \mathrm{pm}$ ) or more than eight hours on any weekend day, and (7) child classified as underweight by a BMI percentile $<5 \%$ adjusted for age and sex.

The study was reviewed and approved by the institutional review board at the University of Southern California and Northeastern University. Dyads were recruited. Mothers provided consent for their own participation and parental consent for their child. Children provided assent. After this process, dyads completed paper and pencil questionnaire measures. A trained research assistant took weight and height measures. Dyads were given additional paper and pencil measures to take home to complete and return to the researchers. Mothers and children each received $\$ 100$ compensation for their time and effort in the first wave of the study.

\section{Measures}

Child BMI. Height and weight were measured using an electronically calibrated digital scale and professional stadiometer. These measures were used to calculate BMI $\left(\mathrm{kg} / \mathrm{m}^{2}\right)$ for mothers and age- and gender-specific body mass index percentiles for children.

Child internalizing symptoms. The Revised Children's Anxiety and Depression Scale (RCADS) ${ }^{\mathbf{5 0}}$ was used to assess child internalizing symptoms. The following subscales were measured: generalized anxiety, major depression, panic disorder, and separation anxiety. Some sample items include: "I feel sad or empty" and "I worry that bad things will happen to me." This 47-item questionnaire utilizes a response scale ranging from 0 (never) to 3 (always) for each item. A total score was calculated with higher scores indicating greater internalizing symptoms. A systematic review and meta-analysis showed that the RCADS has adequate psychometric properties across various assessment settings, languages, and locations. ${ }^{\mathbf{5 1}}$

Child self-esteem. The Rosenberg Self-Esteem (RSE) ${ }^{\mathbf{5}}$ scale was used to assess child global self-esteem. This 10 -item scale asks participants to reflect on their feelings about themselves on a scale ranging from 1 (strongly disagree) to 4 (strongly agree). Sample items include: "I feel that I have a number of good qualities" and "I am able to do things as well as most other people." The RSE has shown good convergent validity for students in high school and college students, men, women, various ethnic groups, and reliability and concurrent validity for high school students. ${ }^{53-54}$ 
Maternal controlling feeding practices. The Child Feeding Questionnaire (CFQ) ${ }^{55}$ utilizes self-reported measures to determine parental beliefs, attitudes, and practices and their effects on child feeding. Restrictive \& Pressure to Eat subscales were used in the current study. The eight-item Restriction Eating subscale assessed restrictive pressures enforced by parents on the type and amount of food a child eats. A sample item is, "I have to be sure that my child does not eat too many high-fat foods." The eight-item Pressure to Eat subscale assesses the frequency of pressuring the child to eat more food. A sample item is, "My child should always eat all of the food on her plate." Mothers indicated on a scale from 1 (disagree) to 5 (agree) as to how much they engaged in various feeding behaviors. Studies found good psychometric properties of the CFQ in Hispanic and non-Hispanic mothers. ${ }^{29,55-56}$

\section{Statistical Analyses}

Analysis of covariance (ANCOVA) models were calculated to examine main effects and two- and three-way interactions among child gender, child age, and mother ethnicity in relation to maternal feeding practices (i.e., restrictive eating and pressure to eat). Age was used as a covariate given developmental influences on maternal feeding practices. ${ }^{49}$ To answer the second research question, bivariate Pearson correlations and descriptive statistics were calculated among maternal feeding practices and BMI- $z$, global internalizing symptoms, and self-esteem. Three hierarchical linear regression analyses were used to examine differences in the effect of maternal feeding practices on internalizing symptoms, self-esteem, and BMI-z, moderated by gender and ethnicity. Two-way interaction terms were created between gender and maternal feeding practices and ethnicity and maternal feeding practices. Age was included as a covariate in regression analyses. In the hierarchical linear regression, the first step included age; the second step included age, gender, ethnicity, and maternal feeding practice main effects; the third step added the interactions between gender and maternal feeding practices and ethnicity and maternal feeding practices. Significance tests were conducted at $p<.05$ level.

\section{RESULTS}

\section{Research Question 1}

There were no significant two- or three-way interactions among child age, child gender, and mother ethnicity in relation to pressure to eat or restriction. Thus, interactions were removed from models. Neither child gender $(p=.96)$ nor mother ethnicity $(p=.52)$ were related to pressure to eat. Older children had mothers who reported greater pressure to eat $(p=.01)$. Neither child gender $(p=.17)$ nor mother ethnicity $(p=.38)$ were related to restriction. Older children had mothers who reported greater restriction $(p=.04)$

\section{Research Question 2}

Bivariate correlations are presented in Table 1. Pressure to eat and restriction were only weakly positively correlated. Pressure to eat was significantly related to lower BMI-z scores and lower child self-esteem scores. Greater restriction was significantly, yet weakly, associated with higher BMI-z scores. There were no associations between maternal controlling feeding practices and global internalizing symptoms.

\begin{tabular}{llllll}
\hline & $\mathbf{1}$ & $\mathbf{2}$ & $\mathbf{3}$ & $\mathbf{4}$ & $\mathbf{5}$ \\
\hline 1. Maternal pressure to eat & - & $.16^{*}$ & $-.41^{* * *}$ & .07 & $-.23^{* *}$ \\
2. Maternal restriction & & - & $.20^{* *}$ & .09 & -.04 \\
3. Child BMI-z & & & - & .12 & -.09 \\
4. Child internalizing symptoms & & & & - & $-.59^{* * *}$ \\
5. Child self-esteem & & & & & - \\
\hline $\boldsymbol{M}$ & 2.39 & 3.03 & 0.52 & 0.58 & 31.88 \\
SD & 1.07 & 0.97 & 1.05 & 1.05 & 5.31 \\
Minimum & 1.00 & 1.00 & -2.63 & 0.00 & 13.00 \\
Maximum & 5.00 & 5.00 & 2.61 & 2.23 & 40.00 \\
Skewness & 0.36 & -0.24 & -0.16 & 1.36 & -0.78 \\
Kurtosis & -0.95 & -0.73 & -0.51 & 2.42 & 0.64 \\
\hline Note. BMI & & & & & \\
\hline
\end{tabular}

Note. $\mathrm{BMI}=$ body mass index. ${ }^{* * *} \mathrm{p}<.001, * * p<.01, * p<.05$

Table 1. Descriptive Statistics among Study Variables. 
Research Question 3

Results of hierarchical linear regression analyses are displayed in Table 2. Older children had fewer internalizing symptoms and better self-esteem across steps. There were no significant statistical interactions among maternal controlling feeding practices and gender or ethnicity in relation to any dependent variables. Further, results showed similar patterns on findings as bivariate correlations.

\begin{tabular}{|c|c|c|c|c|c|c|c|c|c|}
\hline & \multicolumn{3}{|c|}{ BMI-z } & \multicolumn{3}{|c|}{ Internalizing Symptoms } & \multicolumn{3}{|c|}{ Self-Esteem } \\
\hline & $B$ & $\mathrm{SE}$ & $p$ & $B$ & $\mathrm{SE}$ & $p$ & $B$ & $\mathrm{SE}$ & $p$ \\
\hline \multicolumn{10}{|l|}{ Step 1} \\
\hline Child Age & .09 & .08 & .26 & -.76 & .25 & .003 & 1.31 & .44 & .003 \\
\hline \multicolumn{10}{|l|}{ Step 2} \\
\hline Child Age & .02 & .07 & .82 & -.75 & .26 & .01 & 1.00 & .44 & .02 \\
\hline Pressure to eat & -.46 & .06 & $<.001$ & .16 & .23 & .47 & -1.12 & .37 & .003 \\
\hline Restriction & .31 & .07 & $<.001$ & .18 & .25 & .47 & -.10 & .41 & .81 \\
\hline Female & .15 & .13 & .25 & .10 & .47 & .83 & -1.50 & .77 & .05 \\
\hline Hispanic & .34 & .13 & .01 & .82 & .47 & .08 & -.83 & .77 & .28 \\
\hline \multicolumn{10}{|l|}{ Step 3} \\
\hline Child Age & .01 & .07 & .93 & -.76 & .27 & .01 & .99 & .45 & .03 \\
\hline Pressure to eat & -.45 & .11 & $<.001$ & -.26 & .38 & .49 & -.68 & .64 & .29 \\
\hline Restriction & .33 & .12 & .01 & .31 & .42 & .45 & .32 & .71 & .66 \\
\hline Female & .13 & .13 & .31 & .10 & .47 & .83 & -1.47 & .78 & .06 \\
\hline Hispanic & .37 & .13 & .01 & .90 & .47 & .06 & -.91 & .78 & .25 \\
\hline Pressure to eatXFemale & .20 & .13 & .12 & .60 & .45 & .19 & -.70 & .74 & .35 \\
\hline Pressure to eatXHispanic & -.25 & .13 & .05 & .20 & .45 & .66 & -.09 & .74 & .90 \\
\hline RestrictionXFemale & -.11 & .14 & .45 & -.57 & .50 & .26 & .28 & .83 & .74 \\
\hline RestrictionXHispanic & .11 & .14 & .46 & .34 & .50 & .49 & -1.10 & .83 & .19 \\
\hline
\end{tabular}

Table 2. Hierarchical Linear Regressions of Outcomes on Maternal Controlling Feeding Practices, Gender, Ethnicity, and Interactions.

\section{DISCUSSION}

This cross-sectional study examined how maternal controlling feeding practices were associated with weight, global internalizing symptoms, and self-esteem among 8-12-year-old children and how child gender and mother ethnicity may moderate these relationships. There were no differences in reported maternal controlling feeding practices by child gender or mother ethnicity, but older children had mothers who reported greater restriction. This pattern of findings suggests that other factors may be more salient as to which mothers are more likely to use these practices with children of this age range, such as maternal mental health and parenting styles. ${ }^{30,57}$ 
Maternal pressure to eat was associated with lower child BMI z-scores, and maternal restriction was associated with higher child BMI-z scores. Mothers may be more likely to pressure lower weight children to eat more food as they may perceive their child as not getting appropriate nutrition. However, of note, severely underweight children were excluded. Oppositely, mothers may restrict the food intake of higher weight children given the health detriments and social stigma associated with being overweight and obese. Some prior research has shown no associations between maternal controlling feeding practices and BMI among Hispanics ${ }^{23-29}$; however, the current results found that ethnicity did not moderate the significant associations. Given the increasing public health focus on maintaining healthy child weight, ${ }^{43}$ mothers of children, regardless of ethnicity, may become more focused on using controlling feeding practices with children outside of prescribed weight ranges.

Results indicated that pressure to eat and self-esteem scores were negatively correlated. One study showed that higher parental pressure to achieve was related to lower child self-esteem suggesting that undue parental pressure could potentially decrease child self-esteem. ${ }^{59}$ Results of the current study show that this may extend to pressure to eat. Perhaps maternal pressuring reduces a child's feelings of autonomy, which then decreases their self-esteem and confidence to make decisions. ${ }^{60}$

There were no significant relationships among maternal controlling feeding practices and global internalizing symptoms. This is the first study to our knowledge that has examined this relation; however, studies have found associations between maternal controlling feeding practices and child bulimic symptoms and emotional eating scores. ${ }^{20,24}$ It is possible that controlling feeding practices are limited to being associated with eating psychopathology and no other forms of psychopathology. Furthermore, maternal controlling feeding practices may be more detrimental to older adolescents' mental health or only in children at risk for eating disorders. Given the dearth of literature in this area, it is important to conduct more research on these relationships in diverse samples of mother-child dyads.

This study did not find any differences in the association of controlling feeding practices and weight and internalizing symptoms by gender and ethnicity. It is possible that differences between boys and girls may emerge later into adolescence. Continuous exposure to controlling feeding patterns at ages 13-15 has been correlated with unhealthy eating attitudes, such as restriction (more prevalent in boys as compared to girls) and pressuring oneself to eat (more prevalent in girls as compared to boys) ${ }^{61-62}$ Girls are more likely to diet during adolescence, as compared to boys; a past review indicated that 41-66\% adolescent girls diet, and 20$31 \%$ adolescent boys diet, both for the purpose of weight loss. Girls are more likely to diet because of society's perception of the ideal, extremely thin female body. Popular adolescent dieting methods, especially for girls, include crash diets, fasting, slimming tablets, diuretics, and laxatives. Peer pressure, media pressure, and perception of the harmlessness of dieting strategies perpetuate these behaviors. ${ }^{63-64}$ Internalized symptoms starting at a young age become the norm for these adolescents and thus follow them into adulthood. Further, ethnic differences may be more likely to emerge in Hispanic families who are less acculturated to Western society. ${ }^{44}$ More research is needed in adolescents and diverse groups of Hispanic dyads with regard to acculturation.

A limitation of the current study includes a cross-sectional analytic strategy, which limits the ability to infer causation and directionality of effects. This was a community-based sample that was generally well-adjusted and had fewer mental health problems compared to clinical samples. Samples with greater numbers of children with mental health problems may produce differing results. Thus, future studies will be improved by sampling from clinical populations, and future studies should consider examining differences between overt (i.e., controlling a child's food intake in a way that can be detected by the child such as verbally restricting or encouraging eating) versus covert (i.e., controlling a child's food intake in a way that cannot be detected by the child such as placing smaller portions on plates or reducing amounts of snack food available in-home) forms of pressure to eat and restricting, which differ in whether the child can detect the controlling behavior. ${ }^{34,65}$ Overt and covert control can be measured with a 9-item questionnaire that asks about direct and indirect controlling feeding practices. ${ }^{65}$ These constructs are difficult to measure, and further research needs to be done to improve current measures. Future studies may be improved by utilizing more accurate video observational methods, as compared to self-reporting of intake; this may aid in the standardization of measures ${ }^{66}$ Finally, parental stress, home environment, socioeconomic standing, single versus dual-parent households, food scarcity, and cultural versus ethnic differences should be considered in future studies as possible moderators or mediators.

While future studies are necessary to explore the relationships between maternal controlling feeding practices and child weight and internalizing problems, this study provided new insights into these associations and showed that associations are similar across gender and ethnicity.

\section{CONCLUSIONS}

Maternal pressure to eat was correlated with lower child BMI z-scores, and maternal restriction was correlated with higher child BMI-z scores. In general, there were no significant correlations between maternal controlling feeding practices and internalizing symptoms, as well as no significant differences based on gender and ethnicity of a child. However, more maternal pressure to eat was related to lower self-esteem. This is the first study, to our knowledge, that studied the effect of controlling feeding practices 
on global internalizing symptoms and self-esteem. Results provide some evidence that controlling feeding practices may be associated with less self-esteem in middle childhood, but there were no differences in relation to internalizing symptoms. More research will be needed to determine if controlling feeding practices impact mental health into adolescence. Also, results were similar across gender and ethnicity, suggesting that controlling feeding practices do not have a differential impact on mental health and weight in middle childhood. Future studies must be done to further explore the relationship between maternal feeding practices, child weight, and mental health, as well as the effect of gender and ethnicity, particularly in older children and samples with more variability in internalizing symptoms.

\section{ACKNOWLEDGEMENTS}

The author thanks everyone at the USC REACH lab for being so welcoming and for providing advice and suggestions throughout the duration of this project.

\section{REFERENCES}

1. Hruby, A., and Hu, F. B. (2015) The epidemiology of obesity: A big picture, Pharmacoeconomics 33, 673-689. doi:10.1007/s40273 014-0243-x

2. Ludwig, D. S. (2018) Epidemic childhood obesity: Not yet the end of the beginning, Pediatrics 141, e20174078. doi:10.1542/peds.2017-4078

3. Bor, W., Dean, A. J., Najman, J., and Hayatbakhsh, R. (2014) Are child and adolescent mental health problems increasing in the 21st century? A systematic review, Aust N Z J Psychiatry, 48, 606-616. doi:10.1177/0004867414533834

4. Fink, E., Patalay, P., Sharpe, H., Holley, S., Deighton, J., and Wolpert, M. (2015) Mental health difficulties in early adolescence: A comparison of two cross-sectional studies in England from 2009 to 2014, J Adolesc Health 56, 502-507. doi:10.1016/j.jadohealth.2015.01.023

5. Patel, V., Flisher, A. J., Hetrick, S., and Mcgorry, P. (2007) Mental health of young people: A global public-health challenge, Lancet 369, 1302-1313. doi:10.1016/s0140-6736(07)60368-7

6. Bennett, A. C., Brewer, K. C., and Rankin, K. M. (2011) The association of child mental health conditions and parent mental health status among U.S. children, 2007, Maternal Child Health J 16, 1266-1275. doi:10.1007/s10995-011-0888-4

7. Moussavi, S., Chatterii, S., Verdes, E., Tandon, A., Patel, V., and Ustun, B. (2007) Depression, chronic diseases, and decrements in health: Results from the World Health Surveys, Lancet 370, 851-858. doi:10.1016/s0140-6736(07)61415-9

8. Russell-Mayhew, S., McVey, G., Bardick, A., and Ireland, A. (2012) Mental health, wellness, and childhood overweight/obesity, J Obes 2012, 281801. doi:10.1155/2012/281801

9. Canadian Task Force on Preventive Health Care. (2015) Recommendations for growth monitoring, and prevention and management of overweight and obesity in children and youth in primary care, CMAJ 187, 411-421. doi:10.1503/cmaj.141285

10. Payne, L. O., Galloway, A. T., and Webb, R. M. (2011) Parental use of differential restrictive feeding practices with siblings, Int J Pediatr Obes 6, e540-546. doi:10.3109/17477166.2011.575144

11. Farrow, C. V., and Blissett, J. (2008) Controlling feeding practices: Cause or consequence of early child weight?, Pediatrics 121 , e164-e169. doi:10.1542/peds.2006-3437

12. Rodgers, R. F., Paxton, S. J., Massey, R., Campbell, K. J., Wertheim, E. H., Skouteris, H., and Gibbons, K. (2013) Maternal feeding practices predict weight gain and obesogenic eating behaviors in young children: A prospective study, Int J Behav Nutr Phys Act 10, 24. doi:10.1186/1479-5868-10-24

13. Boswell, N., Byrne, R., and Davies, P. S. W. (2018) An examination of children's eating behaviours as mediators of the relationship between parents' feeding practices and early childhood BMIz, Obes Sci Pract. doi:10.1002/osp4.320

14. Pi-Sunyer, F. X. (2002) The Medical Risks of Obesity. Obesity Surgery, 12(S1) doi:10.1007/bf03342140

15. Kearns, K., Dee, A., Fitzgerald, A. P., Doherty, E., \& Perry, I. J. (2014) Chronic disease burden associated with overweight and obesity in Ireland: The effects of a small BMI reduction at population level. BMC Public Health,14(1) doi:10.1186/14712458-14-143

16. Grucza, R. A., Krueger, R. F., Racette, S. B., Norberg, K. E., Hipp, P. R., \& Bierut, L. J. (2010) The Emerging Link Between Alcoholism Risk and Obesity in the United States. Archives of General Psychiatry, 67(12), 1301. doi:10.1001/archgenpsychiatry.2010.155

17. M.S. Faith, K.S. Scanlon, L.L. Birch, L.A. Francis, B. SherryParent-child feeding strategies and their relationships to child eating and weight status Obesity Research, 12 (11) (2004), pp. 1711-1722, doi: 10.1038/oby.2004.212

18. L.L. Birch, J.O. Fisher, K.K. Davison Learning to overeat: Maternal use of restrictive feeding practices promotes girls' eating in the absence of hunger American Journal of Clinical Nutrition, 78 (2) (2003), pp. 215-220, 10.1038/ $\mathrm{nrm} 2621$

19. Smokowski, P.R., Bacallao, M.L., Cotter, K.L. et al. Child Psychiatry Hum Dev (2015) 46: 333. https:// doiorg.libproxy1.usc.edu/10.1007/s10578-014-0474-2

20. Blissett, J., Meyer, C., and Haycraft, E. (2006) Maternal and paternal controlling feeding practices with male and female children, Appetite 47, 212-219. doi:10.1016/j.appet.2006.04.002 
21. Van Strien, T., and Bazelier, F. G. (2007) Perceived parental control of food intake is related to external, restrained and emotional eating in 7-12-year-old boys and girls, Appetite 49, 618-625. doi:10.1016/j.appet.2007.03.227

22. Haycraft, E. L., and Blissett, J. M. (2008) Maternal and paternal controlling feeding practices: reliability and relationships with BMI, Obes 16, 1552-1558. doi:10.1038/oby.2008.238

23. Hughes, S. O., Power, T. G., O’Connor, T. M., Fisher, J. O., and Chen, T. (2016) Maternal feeding styles and food parenting practices as predictors of longitudinal changes in weight status in Hispanic preschoolers from low-income families, $J$ Obes 2016, 1-9. doi:10.1155/2016/7201082

24. Farrow, C. V., Haycraft, E., and Blissett, J. M. (2015) Teaching our children when to eat: How parental feeding practices inform the development of emotional eating — a longitudinal experimental design, Am J Clin Nutr 101, 908-913. doi:10.3945/ajcn.114.103713

25. Darling, K. E., Fahrenkamp, A. J., Ruzicka, E. B., and Sato, A. F. (2018) Controlling feeding practices mediate the association between food insecurity and parent-reported child BMI percentile, Child Care Health 47, 275-288. doi:10.1080/02739615.2017.1337517

26. Gu, C., Warkentin, S., Mais, L. A., and Carnell, S. (2017) Ethnic differences in parental feeding behaviors in UK parents of preschoolers, Appetite 113, 398-404. doi:10.1016/j.appet.2017.03.001

27. Gregory, J. E., Paxton, S. J., and Brozovic, A. M. (2010) Pressure to eat and restriction are associated with child eating behaviours and maternal concern about child weight, but not child body mass index, in 2- to 4-year-old children, Appetite 54, 550-556. doi:10.1016/j.appet.2010.02.013

28. Dovey, T. M., Staples, P. A., Gibson, E. L., and Halford, J. C. (2008) Food neophobia and 'picky/fussy' eating in children: a review. Appetite 50, 181-193. https://doi.org/10.1016/j.appet.2007.09.009

29. Anderson, C. B., Hughes, S. O., Fisher, J. O., and Nicklas, T. A. (2005) Cross-cultural equivalence of feeding beliefs and practices: The psychometric properties of the child feeding questionnaire among Blacks and Hispanics, Prev Med 41, 521-531. doi:10.1016/j.ypmed.2005.01.003

30. Goulding, A. N., Rosenblum, K. L., Miller, A. L., Peterson, K. E., Chen, Y., Kaciroti, N., and Lumeng, J. C. (2014) Associations between maternal depressive symptoms and child feeding practices in a cross-sectional study of low-income mothers and their young children, Int J Behav Nutr Phys Act 11, 75. doi:10.1186/1479-5868-11-75

31. Herpertz-Dahlmann, B., Wille, N., Hölling, H., Vloet, T. D., Ravens-Sieberer, U., and BELLA Study Group. (2008) Disordered eating behaviour and attitudes, associated psychopathology and health-related quality of life: results of the BELLA study, Eur Child Adolesc Psychiatry 17, 82-91. doi:10.1007/s00787-008-1009-9

32. Bauer, K. W., Hearst, M. O., Escoto, K., Berge, J. M., \& Neumark-Sztainer, D. (2012) Parental employment and work-family stress: Associations with family food environments. Social Science \& Medicine, 75(3), 496-504. doi: 10.1016/j.socscimed.2012.03.026.

33. Birch, L., Fisher, J., Grimm-Thomas, K., Markey, C., Sawyer, R., and Johnson, S. (2001) Confirmatory factor analysis of the Child Feeding Questionnaire: A measure of parental attitudes, beliefs and practices about child feeding and obesity proneness, Appetite 36, 201-210. doi:10.1006/appe.2001.0398

34. Jennings, K. M., Loth, K. A., Tate, A. D., Miner, M. H., \& Berge, J. M. (2019) Application of latent profile analysis to define subgroups of parenting styles and food parenting practices. Appetite, 139, 8-18. doi: 10.1016/j.appet.2019.04.001

35. Brown, K. A., Ogden, J., Vögele, C., and Gibson, E. L. (2008) The role of parental control practices in explaining childrens diet and BMI, Appetite 50, 252-259. doi:10.1016/j.appet.2007.07.010

36. Fisher, J., \& Birch, L. (1999) Restricting Access to Foods and Childrens Eating. Appetite, 32(3), 405-419. doi: 10.1006/appe.1999.0231

37. Wertheim, E.H., Mee, V. \& Paxton, S.J. Relationships Among Adolescent Girls' Eating Behaviors and Their Parents' WeightRelated Attitudes and Behaviors. Sex Roles 41, 169-187 (1999) https://doi.org/10.1023/A:1018850111450

38. Cardel, M., Willig, A. L., Dulin-Keita, A., Casazza, K., Beasley, T. M., \& Fernández, J. R. (2012) Parental feeding practices and socioeconomic status are associated with child adiposity in a multi-ethnic sample of children. Appetite, 58(1), 347-353. doi: 10.1016/j.appet.2011.11.005

39. Wehrly, S. E., Bonilla, C., Perez, M., and Liew, J. (2014) Controlling parental feeding practices and child body composition in ethnically and economically diverse preschool children, Appetite 73, 163-171. doi:10.1016/j.appet.2013.11.009

40. Berge, J. M., Tate, A., Trofholz, A., Loth, K., Miner, M., Crow, S., \& Neumark-Sztainer, D. (2018) Examining variability in parent feeding practices within a low-income, racially/ethnically diverse, and immigrant population using ecological momentary assessment. Appetite, 127, 110-118. doi: 10.1016/j.appet.2018.04.006

41. Cachelin, F. M., \& Thompson, D. (2013) Predictors of maternal child-feeding practices in an ethnically diverse sample and the relationship to child obesity. Obesity, 21(8), 1676-1683. doi: 10.1002/oby.20385

42. Caprio, S., Daniels, S. R., Drewnowski, A., Kaufman, F. R., Palinkas, L. A., Rosenbloom, A. L., ... Kirkman, M. S. (2008) Influence of Race, Ethnicity, and Culture on Childhood Obesity: Implications for Prevention and Treatment. Obesity, 16(12), 2566-2577. doi: 10.1038/oby.2008.398 
43. Shea, M., Cachelin, F., Uribe, L., Striegel, R. H., Thompson, D., \& Wilson, G. T. (2012) Cultural Adaptation of a Cognitive Behavior Therapy Guided Self-Help Program for Mexican American Women with Binge Eating Disorders. Journal of Counseling \& Development, 90(3), 308-318. doi: 10.1002/j.1556-6676.2012.00039.x

44. New, C., Xiao, L., and Ma, J. (2013) Acculturation and overweight-related attitudes and behavior among obese Hispanic adults in the United States, Obes 21, 2396-2404. doi: 10.1002/oby.20146

45. Jain, A., Sherman, S. N., Chamberlin, D. L. A., Carter, Y., Powers, S. W., \& Whitaker, R. C. (2001) Why Don't Low-Income Mothers Worry About Their Preschoolers Being Overweight? Pediatrics, 107(5), 1138-1146. doi: 10.1542/peds.107.5.1138

46. Martinez, S. M., Rhee, K. E., Blanco, E., \& Boutelle, K. (2016) Latino mothers' beliefs about child weight and family health. Public Health Nutrition, 20(6), 1099-1106. doi: 10.1017/s1368980016002962

47. Bruss, M. B., Morris, J. R., Dannison, L. L., Orbe, M. P., Quitugua, J. A., \& Palacios, R. T. (2005) Food, Culture, and Family: Exploring the Coordinated Management of Meaning Regarding Childhood Obesity. Health Communication, 18(2), 155-175. doi: $10.1207 /$ s15327027bc1802_4

48. Blissett, J., Meyer, C., \& Haycraft, E. (2006) Maternal and paternal controlling feeding practices with male and female children. Appetite, 47(2), 212-219. doi: 10.1016/j.appet.2006.04.002

49. Bassett, R., Chapman, G. E., \& Beagan, B. L. (2008) Autonomy and control: The co-construction of adolescent food choice. Appetite, 50(2-3), 325-332. doi:10.1016/j.appet.2007.08.009

50. Chorpita, B. F., Yim, L., Moffitt, C., Umemoto, L. A., and Francis, S. E. (2000) Assessment of symptoms of DSM-IV anxiety and depression in children: A revised child anxiety and depression scale, Behav Res Ther 38, 835-855. doi:10.1016/S00057967(99)00130-8

51. Piqueras, J. A., Martín-Vivar, M., Sandin, B., Luis, C. S., and Pineda, D. (2017) The Revised Child Anxiety and Depression Scale: A systematic review and reliability generalization meta-analysis, J Affect Disord 218, 153-169. doi:10.1016/j.jad.2017.04.022

52. Rosenberg, M. (1965) Rosenberg self-esteem scale (RSE) Acceptance and commitment therapy. Measures package, 61, 52.

53. Ciarroch, J., and Bilich, L. (2006) Acceptance and Commitment Therapy. Measures Package Process measures of potential relevance to ACT(Publication) School of Psychology University of Wollongong.

54. Robins, R. W., Hendin, H. M., \& Trzesniewski, K. H. (2001) Measuring global self-esteem: Construct validation of a singleitem measure and the Rosenberg Self-Esteem Scale, Pers Soc Psychol Bull 27, 151-161. doi:10.1177/0146167201272002

55. Birch, L., Fisher, J., Grimm-Thomas, K., Markey, C., Sawyer, R., and Johnson, S. (2001) Confirmatory factor analysis of the Child Feeding Questionnaire: A measure of parental attitudes, beliefs and practices about child feeding and obesity proneness, Appetite 36, 201-210. doi:10.1006/ appe.2001.0398

56. Nowicka, P., Sorjonen, K., Pietrobelli, A., Flodmark, C., and Faith, M. S. (2014) Parental feeding practices and associations with child weight status. Swedish validation of the Child Feeding Questionnaire finds parents of 4-year-olds less restrictive, Appetite 81, 232-241. doi:10.1016/j.appet.2014.06.027

57. Blissett, J., and Haycraft, E. (2008) Are parenting style and controlling feeding practices related?, Appetite 50, $477-485$. doi:10.1016/j.appet.2007.10.003

58. Ballard, R. M., Rodgers, A. B., Khan, L. K., Orleans, C. T., Arkin, E., and Phillips, T. M. (2018) Developing a partnership for change: The National Collaborative on Childhood Obesity Research, Am J Prev Med 54, 465-474. doi:10.1016/j.amepre.2017.12.001

59. Eskilson, A., Wiley, M., Muehlbauer, G., and Dodder, L. (1986) Parental Pressure, Self-Esteem and Adolescent Reported Deviance: Bending the Twig Too Far. ProQuest, 21(83), PMID: 3812058

60. Frederick, C. M., and Grow, V. M. (1996) A mediational model of autonomy, self-esteem, and eating disordered attitudes and behaviors, Psychol Women Q 20, 217-228. doi:10.1111/j.1471-6402.1996.tb00468.x

61. Haycraft, E., Goodwin, H., \& Meyer, C. (2014) Adolescents Level of Eating Psychopathology Is Related to Perceptions of Their Parents Current Feeding Practices. Journal of Adolescent Health, 54(2), 204-208. doi: 10.1016/j.jadohealth.2013.08.007

62. Hautala, L. A., Junnila, J., Helenius, H., Väänänen, A.-M., Liuksila, P.-R., Räihä, H., Välimäki, R., Saarijärvi, S. (2008) Towards understanding gender differences in disordered eating among adolescents. Journal of Clinical Nursing, 17(13), 1803-1813. doi: 10.1111/j.1365-2702.2007.02143.x

63. Grigg, M., Bowman, J., \& Redman, S. (1996) Disordered eating and unhealthy weight reduction practices among adolescent females. Preventive Medicine, 25(6), 748-756. doi: 10.1006/pmed.1996.0115

64. Whyte, H., Findlay, S. M., \& Canadian Paediatric Society, Adolescent Health Committee. (2004) Dieting in adolescence, Paediatrics \& Child Health, 9, 487-491, https:// doi.org/10.1093/pch/9.7.487

65. Nowicka, P., Flodmark, C. E., Hales, D., and Faith, M. S. (2014) Assessment of parental overt and covert control of child's food intake: A population-based validation study with mothers of preschoolers, Eat Behav 15, 673-678. doi:10.1016/j.eatbeh.2014.10.001

66. O'Connor, T. M., Mâsse, L. C., Tu, A. W., Watts, A. W., Hughes, S. O., Beauchamp, M. R., Baranowski, T., Pham, T., Berge, J. M., Fiese, B., Golley, R., Hingle, M., Kremers, S. P. J., Rhee, K. E., Skouteris, H., \& Vaughn, A. (2017) Food parenting 
practices for 5 to 12 year old children: a concept map analysis of parenting and nutrition experts input. International Journal of Behavioral Nutrition and Physical Activity, 14, 122. https://psycnet.apa.org/record/2017-40559-001

\section{ABOUT STUDENT AUTHOR}

Paulina Mozdzierz will graduate in May 2021 with a bachelor's degree in Health Promotion and Disease Prevention and a minor in Business Economics. She plans on enrolling in a physician assistant program after graduating.

\section{PRESS SUMMARY}

Mothers may attempt to control their children's food intake in order to make the child gain or lose weight. The purpose of this study was to determine if these practices have an association with weight and mental health of children. Results were based on 202 pairs of mothers and their children. The following findings were determined as part of the results: mothers pressuring their children to eat was related to a lower child body mass, restricting children from eating certain foods was related to a higher child body mass, and mothers pressuring their children to eat was related to lower child self-esteem. 


\title{
Synthesis of Graphene Oxide Enhanced Agar Composites: A Biocompatible Photo-catalyst for Degradation of Organic Dyes
}

\author{
Shreyas Dindorkar*a, Jaymin Mistry ${ }^{a}$, Jayesh Hire ${ }^{a}$, Khushi Jain ${ }^{b}$, Nandini Khona ${ }^{b}$, Shreya Peddakolmi ${ }^{b}$ \& Paresh More ${ }^{a b}$ \\ a Department of Chemistry, K. E. T's, Vinayak Ganesh Vare College (Autonomous), Mulund, Mumbai, India \\ ${ }^{b}$ Department of Biotechnology, K. E. T's, Vinayak Ganesh Vaze College (Autonomous), Mulund, Mumbai, India
}

bttps:/ / doi.org/10.33697/ajur.2020.025

Students:shreyasdindorkar@gmail.com*,jayminmistry2000@gmail.com,jayesh.hire456@gmail.com,jainkhushi2812@gmail.com, khona.nandini1805@gmail.com,peddakolmis@gmail.com

Mentor:paresh.m34@gmail.com

\begin{abstract}
Herein we report the synthesis of graphene oxide-based agar composites using a solution casting method. Graphene oxide was synthesized by modified Hummer's method and was characterized using X-ray diffraction (XRD) and Raman spectroscopy. The graphene oxide-based agar composites were characterized using X-ray diffraction (XRD) and UV-visible spectroscopy. Optical band gap obtained from the Tauc plot showed that the composites could be used in the photodegradation of dyes. The synthesized composite material was checked for its practical applicability in the degradation of methylene blue dye under solar irradiation; with an increase in the concentration of graphene oxide, catalyst, and $\mathrm{H}_{2} \mathrm{O}_{2}$, the rate constant increases. The rate constant was found to be inversely proportional to the concentration of methylene blue dye. Dosage of graphene oxide was found to be the most prominent factor in increasing the rate of photodegradation. It is clear from the data for the reaction system that the degradation reaction follows pseudo-first-order kinetics.
\end{abstract}

\section{KEYWORDS}

Composites; Ultra-sonication; Photodegradation; Methylene Blue; XRD; Graphene Oxide; Kinetics; Biocompatibility

\section{INTRODUCTION}

Dyes are an important class of synthetic organic compounds used in many industries, especially textiles. Consequently, they have become common industrial environmental pollutants during their synthesis and later during fiber dyeing. ${ }^{1}$ The harmful organic substances generated from chemical, textile, and dye industries are highly polluting the water resources and producing serious problems to all life and their environment. Organic dyes are toxic, mutagenic, and carcinogenic. ${ }^{2}$ Around $10-20 \%$ of the dyes are lost during the dyeing process, and hence, the removal of textile dyes from contaminated water bodies has become a concern worldwide. ${ }^{3}$ The natural photosynthesis cycle process is also affected by the discharge of the textile industry effluents into the environment. ${ }^{4}$ If allowed to flow in drains and rivers, it affects the quality of drinking water in hand pumps, rivers, lakes, and potable reservoirs, making them unfit for human consumption. It is important to remove these pollutants from the wastewaters before their final disposal. Hence, there is a need to address the pollution problem immediately. Due to the chemical and biological stability, the removal of the toxic dyes by natural degradation processes is difficult. ${ }^{5-8}$ The effective removal of these dyes before discharge or their conversion into useful or less harmful products is of great importance. Various attempts are being made worldwide to address this issue by synthesizing novel materials for degradation or conversion of dyes into less harmful products. However, many of these materials, such as $\mathrm{TiO}_{2}$, are toxic to living communities. Replacement of such materials with biodegradable or biocompatible ones is the most attractive yet simple strategy and has received much attention in the last few years. ${ }^{9-11}$

Graphene oxide (GO), a derivative of graphene, is in use for about one and a half centuries. It is a two dimensional (2-D) covalently bonded and oxygen-rich carbon skeletal. Because of its large surface area, excellent strength, and reactive oxygenated groups spread over its surface, it has recently attracted attention in various fields such as optoelectronics, energy storage, catalysis, thermoelectric devices, tissue engineering, and drug delivery. ${ }^{12-16}$ The functionalized surface of graphene oxide with reactive oxygenated species distinguishes it from graphene and has attracted scientists towards its valuable applications in chemistry (Figure 1). This oxygen-rich nature of graphene oxide is responsible for its easy dispersibility in water. This is the most important property of graphene oxide as it enables one to dope it into the polymer and ceramic matrices. Moreover, it also shows excellent dispersibility in most of the organic solvents such as $\mathrm{N}, \mathrm{N}$-dimethylformamide. ${ }^{17}$ Hence, graphene oxide is used in the wet preparation experiments in both aqueous media as well as in organic media. The electrical, mechanical, catalytic properties of 
ceramics, polymers, and composites can be enhanced by doping them with graphene oxide. It was also found that the mechanical strength, electrical conductivity of polymers, more specifically ceramics, were increased to many folds when doped with graphene oxide. ${ }^{18-19}$ Graphene oxide is non-toxic at low concentrations, and the toxicity can be further reduced by surface modification with biocompatible material. ${ }^{20}$

Biodegradable polymers have expanded the idea to tackle the growing environmental problem associated with the use of synthetic polymers leading to plastic waste. The hunt for cost-effective, environmentally friendly materials has led to the development of different biodegradable plastics. ${ }^{21}$ Agar is a jelly-like substance obtained from red marine algae and possesses distinctive characteristics such as biocompatibility, solubility in water, biodegradability, and low cost. It gets its gelling properties from an unbranched polysaccharide, which is obtained from the cell walls of some species of red algae, primarily from Gelidiaceae and Gracilaria. It has a wide range of applications, most commonly in the pharmaceutical and medical industries. ${ }^{22}$

There are numerous reports in the literature where graphene oxide has been combined with other materials to make nanocomposites for application in the degradation of industrial dyes. M. Bakhtir Azim et al. synthesized GO-TiO 2 nanocomposites for degradation of methylene blue (MB), which showed around 89\% degradation. ${ }^{23}$ Humaira Seema et al. had reported the synthesis of $\mathrm{GO}-\mathrm{SnO}_{2}$ composites which gave complete degradation of methylene blue under solar light. ${ }^{24} \mathrm{Yanhui}$ Li et al. synthesized GO-Agar aerogel for removal of methylene blue dye, which exhibited an excellent adsorption capacity of 578 $\mathrm{mg} / \mathrm{g}$ and reusability up to 3 cycles. ${ }^{25}$ Gelatin/PVA-GO biocomposites have been reported by L. E. Crica et al., which showed remarkable improvements in mechanical properties. ${ }^{26}$

Methylene blue is an industrial dye that is commonly used for dying fabrics and in staining biological samples. Apart from industries, it finds application as medication. It is mainly used to treat methemoglobinemia and methemoglobin levels that are greater than $30 \%$. However, if present in high concentrations, it is extremely hazardous to human health. Direct contact of methylene blue with eyes causes extreme irritation, and it is highly toxic by oral and intravenous routes. It is also combustible in powder form and releases highly toxic gases such as nitrogen oxides. Therefore, proper treatment is necessary before releasing such dyes into water bodies.

Verma et al. and their group have reported biocompatible graphene and graphene oxide doped agar composites, which exhibited excellent tensile strength, thermal properties, and water resistance property. ${ }^{27}$ The present work is on the same line as Verma $e t$ al. and aims to synthesize biocompatible Agar@GO composites by a simple solution casting method. Various analytical techniques such as XRD, Raman, UV-Visible were utilized. Further, the composites were tested for photocatalytic degradation of methylene blue.

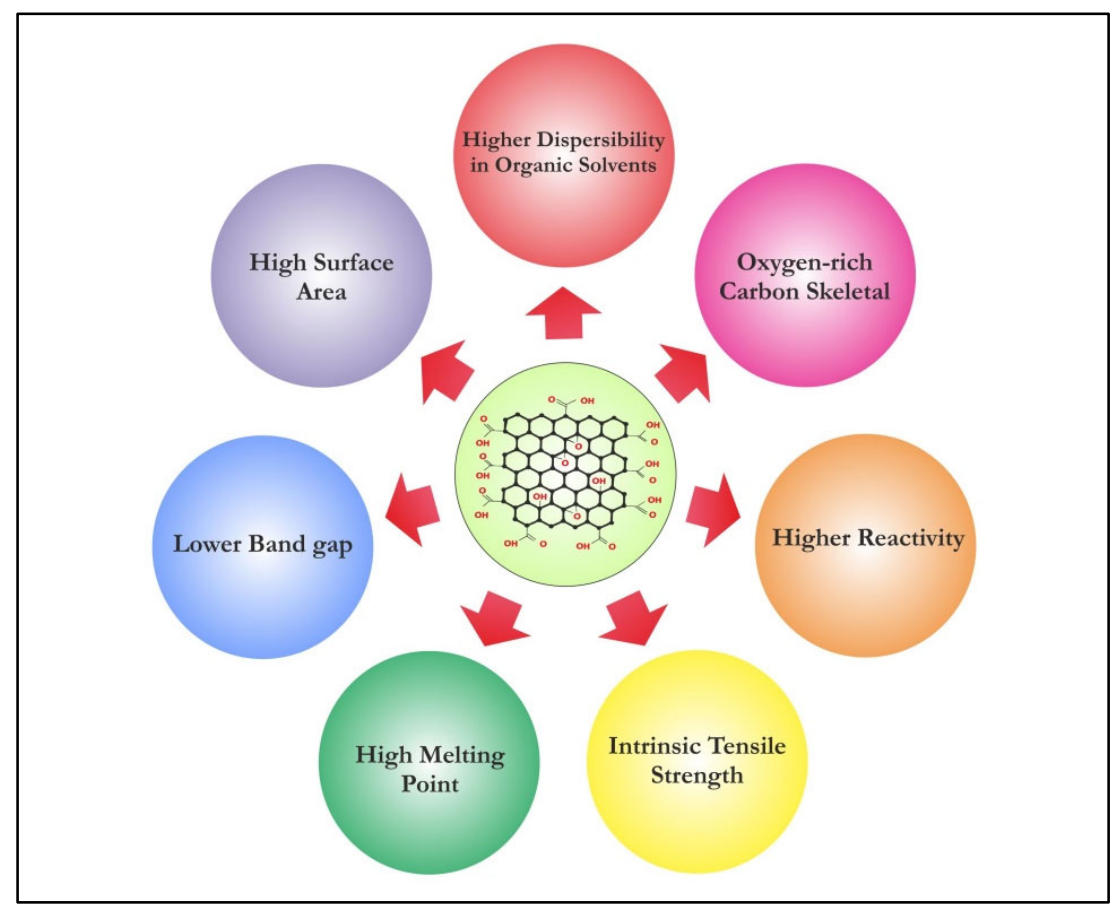

Figure 1. Properties of graphene oxide. 


\section{EXPERIMENTAL}

\section{Materials:}

Finely powdered graphite was purchased from LOBA Pvt. Ltd. Gelatin Agar powder was purchased from Weiss mill. Potassium permanganate $\left(\mathrm{KMnO}_{4}\right)$, sulfuric acid $\left(\mathrm{H}_{2} \mathrm{SO}_{4}, 98 \%\right)$, hydrochloric acid $(\mathrm{HCl}, 10 \%)$, hydrogen peroxide $\left(\mathrm{H}_{2} \mathrm{O}_{2}, 30 \%\right)$, sodium nitrate $\left(\mathrm{NaNO}_{3}\right)$ were of the analytical grade and were used as received.

\section{Synthesis of Graphene oxide:}

Graphene oxide was synthesized by modified Hummer's method. ${ }^{28} 2.0 \mathrm{~g}$ of finely powdered graphite and $1.0 \mathrm{~g}$ of sodium nitrate $\left(\mathrm{NaNO}_{3}\right)$ were added to $\mathrm{H}_{2} \mathrm{SO}_{4}(46 \mathrm{ml} ; 98 \%)$. The mixture was subjected to constant stirring for 1 hour. $6.0 \mathrm{~g}$ of analytical grade $\mathrm{KMnO}_{4}$ was then added gradually to the mixture. The temperature of the reaction system was maintained at $20^{\circ} \mathrm{C}$ using an ice bath. After addition, the mixture was stirred constantly for 12 hours at $35^{\circ} \mathrm{C}$ using a magnetic stirrer. The solution thus obtained was diluted with $500 \mathrm{ml}$ distilled water under constant stirring. Dilution was followed by the addition of $5.00 \mathrm{ml} \mathrm{H}_{2} \mathrm{O}_{2}(30 \%)$. The resulting product was washed with distilled water to remove the traces of acid, followed by washing with absolute alcohol. The product was dried at $60^{\circ} \mathrm{C}$ for 2 hours and stored in a vial for further experiments.

\section{Synthesis of Agar@GO composites:}

Agar@GO composites were synthesized by a simple solution casting method.20 To prepare 5\% (w/w) Agar@GO composites, 5.0 mg of graphene oxide powder were dispersed in 10\% absolute alcohol and subjected to ultra-sonication for 30 min. Agar solution was prepared by dissolving $100.0 \mathrm{mg}$ of agar powder in distilled water at $60^{\circ} \mathrm{C}$ followed by ultrasonication for $30 \mathrm{~min}$. Both the solutions were then mixed and ultra-sonicated at $60^{\circ} \mathrm{C}$ for $60 \mathrm{~min}$. The adduct so formed was then allowed to cool down at room temperature to obtain jelly-like material, which was air-dried and pulverized to obtain the composites. Similarly, composites with $10 \%, 20 \%$, and $25 \%$ of graphene oxide (w/w) were synthesized and studied for further applications.

\section{Characterization:}

The structural properties of the synthesized graphene oxide and Agar@GO composites were investigated by X-ray diffraction (XRD) technique at room temperature with Xpert pro MPD X-ray diffractometer with $\mathrm{Cu}-\mathrm{K} \alpha$ radiation $(\lambda-1.5405 \AA$ ). Diffraction patterns were recorded at a scan speed of $0.3^{\circ}$ at $40 \mathrm{KV} / 30 \mathrm{~mA}$. Raman spectroscopy was carried out using RENISHAW micro Raman system with power $5 \mathrm{Mw}$ to confirm the formation of graphene oxide. The optical properties of composites and photodegradation of methylene blue dye were studied using a UV-visible spectrophotometer (Shimadzu 1800) with a scanning interval of $0.5 \mathrm{~nm}$ from $200 \mathrm{~nm}$ to $800 \mathrm{~nm}$.

\section{Photodegradation Experiment:}

A simple photodegradation experiment was carried out for the degradation of methylene blue dye under sunlight to investigate the photocatalytic activity of composites. $100 \mathrm{ppm}$ stock solution of the dye was prepared by dissolving $0.1 \mathrm{~g}$ of methylene blue (molecular weight - $319.85 \mathrm{~g} / \mathrm{mol}$ ) in $1000 \mathrm{ml}$ of distilled water. $50.00 \mathrm{ml} 20 \mathrm{ppm}$ solution of dye was then prepared using the stock solution. To the $50.00 \mathrm{ml}$ of the above dye solution, the desired amounts of Agar@GO composites were added, followed by the addition of oxidizing agent $\mathrm{H}_{2} \mathrm{O}_{2}$. The mixture was then exposed to irradiation with occasional stirring. Aliquots of the irradiating mixture were taken at a constant interval of $10 \mathrm{~min}$ and were analyzed by a UV-visible spectrophotometer. The effect of various reaction parameters viz. amount of Agar@GO composites, the concentration of the dye solution, and the amount of $\mathrm{H}_{2} \mathrm{O}_{2}$ was investigated.

\section{RESULTS}

1. X-ray Diffraction and Raman Spectroscopy:

The X-ray diffractogram of chemically synthesized graphene oxide is shown in Figure 2a. Along with an intense peak around $2 \theta$

$=10^{\circ}$, a small hump appeared at $2 \theta=43.1^{\circ}$. No peak was observed around $2 \theta=26^{\circ}$, which indicates the complete conversion of graphite into graphene oxide. ${ }^{29}$ The Raman spectra recorded for graphene oxide (Figure $2 \mathbf{b}$ ) shows two bands at $1349 \mathrm{~cm}^{-1}$ and $1599 \mathrm{~cm}^{-1}$. They are D and G bands, respectively, which arise due to $\mathrm{sp}^{2}$ hybridization of carbon atoms. X-ray diffraction patterns confirmed changes in the chemical structure of agar after the incorporation of graphene oxide (Figure 2c). Agar shows two intense $2 \theta$ peaks at $12.56^{\circ}$ and $19.21^{\circ}$ due to its semi-crystalline nature..$^{30} \mathrm{~A}$ slightly intensified peak is observed at $12.56^{\circ}$ along with a slightly broader $2 \theta$ peak at $43.13^{\circ}$. 

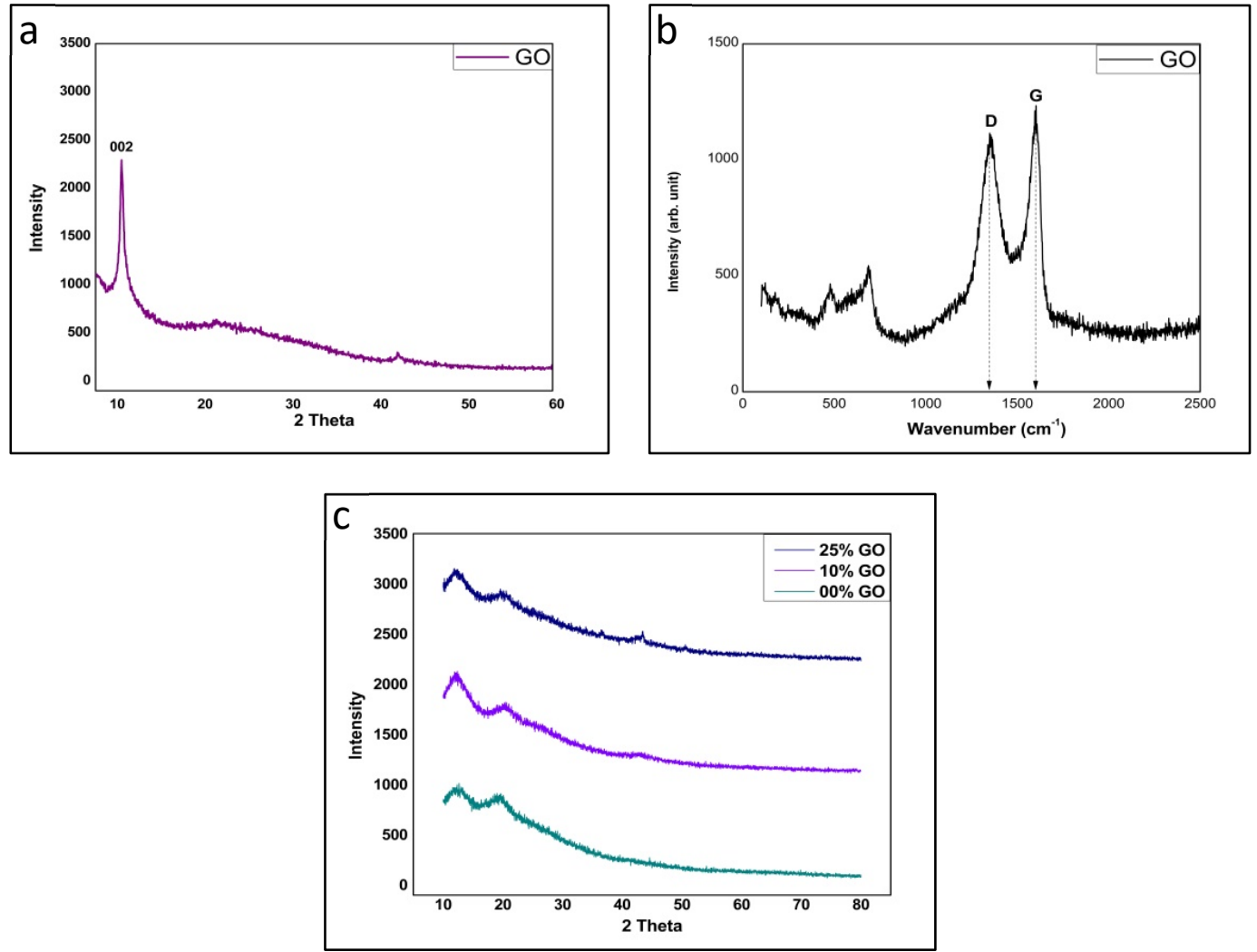

Figure 2. a) XRD pattern of GO b) Raman Spectra of GO c) XRD patterns of Agar@GO composites with varying concentration of GO (GO - graphene oxide).

\section{Optical Properties:}

The synthesized Agar@GO composites were analyzed for optical properties using a UV-visible spectrophotometer. The UVVisible spectrum of the composites was monitored from $200 \mathrm{~nm}$ to $800 \mathrm{~nm}$ by dissolving a certain amount of composites in distilled water. This provided the first confirmation for the formation of Agar@GO composites. Figure 3a depicts the plot of $\%$ Transmittance vs. wavelength $(\mathrm{nm})$. For agar, almost constant transmittance was observed in the visible range, whereas a sudden decrease was observed in the UV range of the spectrum. The sudden decrease in \% Transmittance $(\sim 10 \%)$ is observed in the case of composites and is constant in both UV as well as in the visible region ${ }^{28}$. High \% Transmittance was observed $(\sim 94 \%)$ in the case of agar due to its low concentration. The optical band gap was calculated from UV-visible spectra by Tauc plot method $^{31}$ (Figure 3b). Extrapolating of the straight line in the plot of $(\alpha h v)^{2}$ vs. Energy (hv) gives the value of the optical band gap of composites, which was found to be $3.58 \mathrm{eV}$. Normally lower the value of band gap, the higher is the photocatalytic activity. The observed value (i.e., $3.58 \mathrm{eV}$ ) is sufficiently high and indicates that the energy requirement for excitation of electrons in the composites cannot be fulfilled in the visible region but requires a slightly higher amount of energy (i.e., corresponding to UVVisible region). 

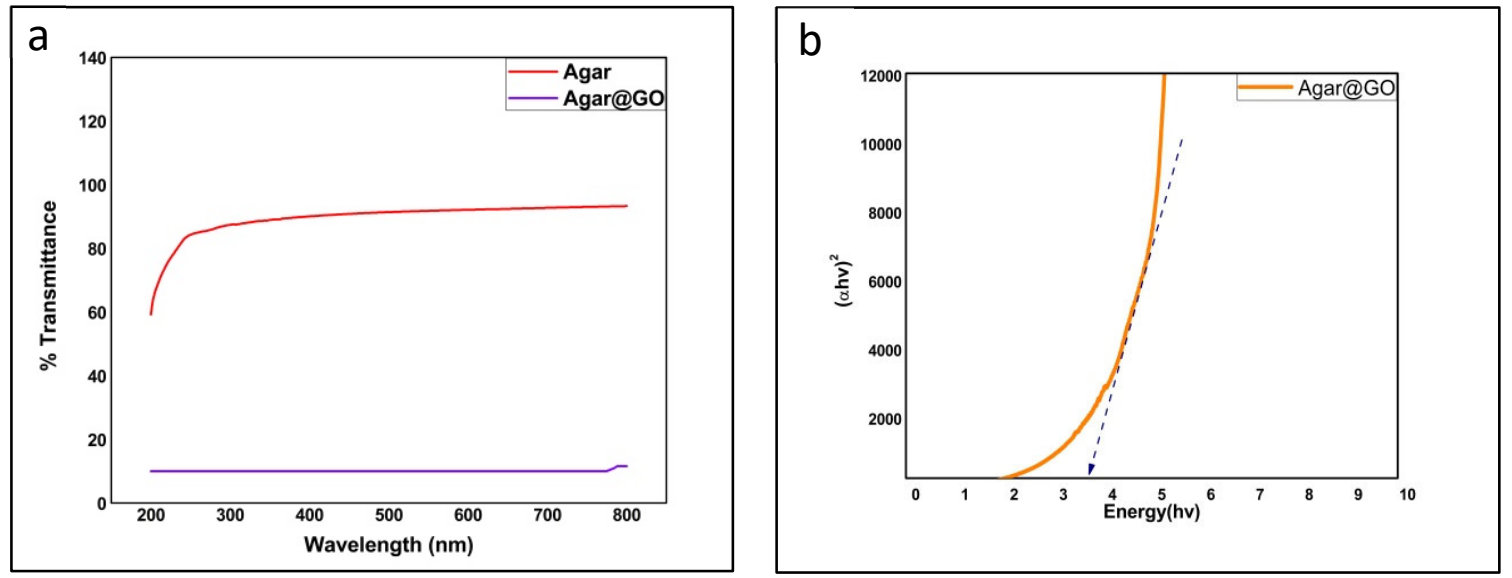

Figure 3. a) \% Transmittance of Agar@GO composites with 25\% (w/w) of GO b) Tauc Plot for Agar@GO composites with 25\% (w/w) of GO (GO graphene oxide).

\section{Photodegradation Experiment:}

The ability of the Agar@GO composites was investigated for the degradation of methylene blue dye under solar irradiation. The degradation was monitored using a UV-visible spectrophotometer. The spectrum showed a gradual decrease in the absorption band at $664 \mathrm{~nm}$ and $292 \mathrm{~nm}$ (Figure 4). Systematic investigation of the effect of reaction parameters on the rate of the reaction was carried out.

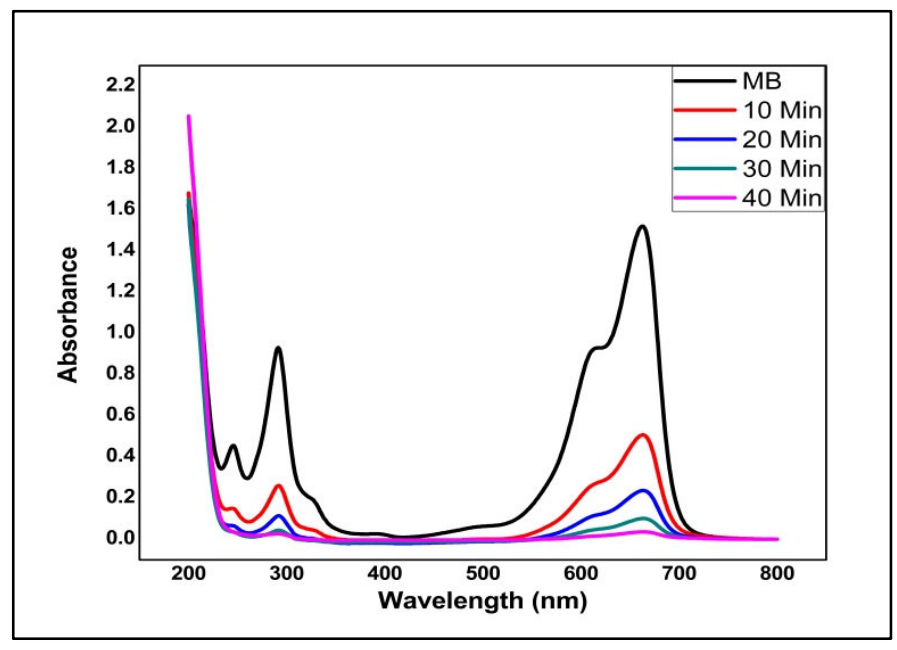

Figure 4. UV-Visible Spectrum of MB Degradation (MB - methylene blue).

\section{Control Experiment:}

Various control experiments were carried out using Agar@GO composites for the degradation of methylene blue under sunlight. The data so obtained from the analysis of the UV-Visible spectrum is depicted in Figure 5. Negligible degradation was observed in the absence of composites and $\mathrm{H}_{2} \mathrm{O}_{2}(\mathrm{MB}+\mathrm{h} v)$. A slight increase in percent degradation was observed after the addition of $\mathrm{H}_{2} \mathrm{O}_{2}$ in the presence of light $\left(\mathrm{MB}+\mathrm{H}_{2} \mathrm{O}_{2}+\mathrm{h} v\right)$ and also in the absence of light $\left(\mathrm{MB}+\mathrm{H}_{2} \mathrm{O}_{2}+\right.$ dark $)$. After the addition of Agar@GO composites (MB + $\mathrm{H}_{2} \mathrm{O}_{2}+$ Agar@GO + hv), almost 100\% degradation was observed. The rate constant was also maximized in this case. This indicates that the composites are responsible for driving the reaction at a faster rate. 


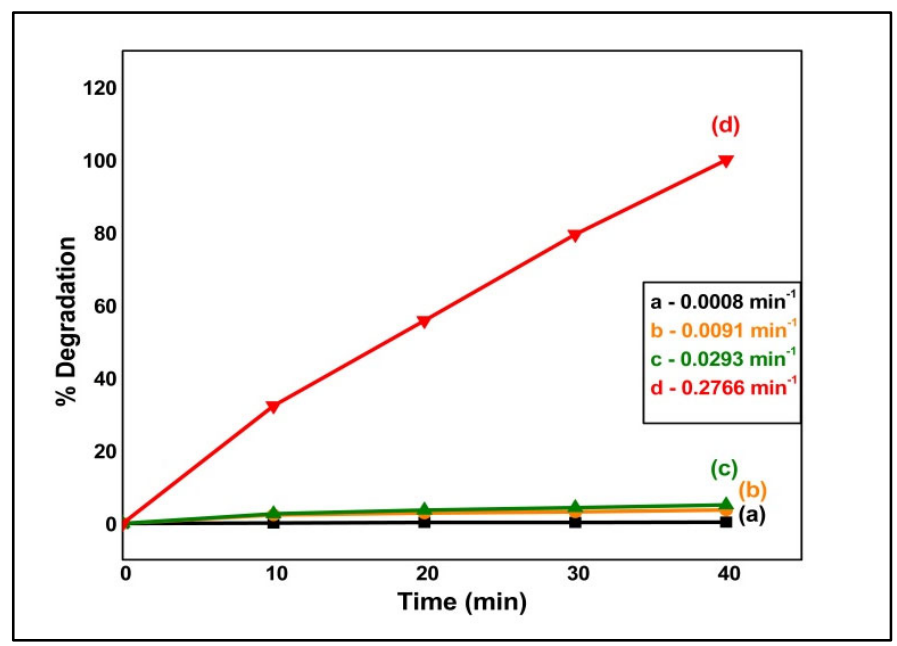

Figure 5. Control Experiment of methylene blue degradation having conditions a) $\left.\left.\mathrm{MB}+\mathrm{h} v \mathrm{~b}) \mathrm{MB}+\mathrm{H}_{2} \mathrm{O}_{2}+\mathrm{dark}_{\mathrm{c}}\right) \mathrm{MB}+\mathrm{H}_{2} \mathrm{O}_{2}+\mathrm{h} \nu \mathrm{d}\right) \mathrm{MB}+\mathrm{H}_{2} \mathrm{O}_{2}+$ Agar@GO + hv (MB - methylene blue).

\section{Kinetics of Reaction:}

Reaction kinetics plays a crucial role in the investigation of reaction systems and their mechanisms. The direction of the reaction can be studied using reaction kinetics. To study the kinetics, the reaction mixtures were analyzed for absorbance band at $664 \mathrm{~nm}$ after regular intervals of $10 \mathrm{~min}$ in the range of $200 \mathrm{~nm}$ to $800 \mathrm{~nm}$. A plot of $\ln \left(\mathrm{C}_{t} / \mathrm{C}_{0}\right)$ vs. Time (t) in min was plotted for each system to obtain the rate constant whose values are given in Table 1. The rate constant was found to be highest in the presence of $\mathrm{H}_{2} \mathrm{O}_{2}$, Agar@GO, and sunlight. For an optimized system, percent degradation after 10, 20, 30, and 40 min was observed to be $32.34 \%, 55.79 \%, 79.51 \%$, and $99.97 \%$, respectively (Figure 6).

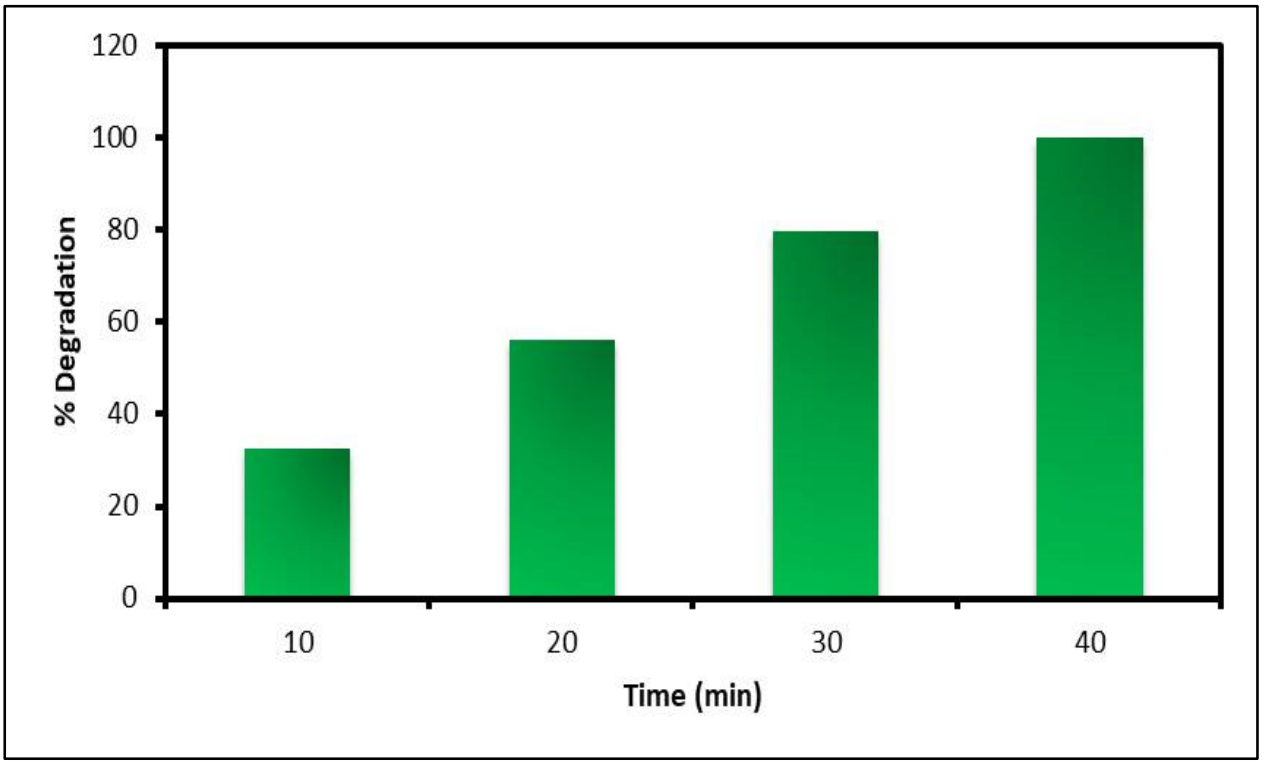

Figure 6. \%Degradation vs. Time (min) for an optimized system. 


\begin{tabular}{|c|c|c|c|c|c|c|c|}
\hline \multicolumn{2}{|c|}{ GO Variation } & \multicolumn{2}{|c|}{ Catalyst Variation } & \multicolumn{2}{|c|}{ Dye Concentration } & \multicolumn{2}{|c|}{$\mathbf{H}_{2} \mathbf{O}_{2}$ Variation } \\
\hline$\%(w / w)$ & $\mathrm{k}\left(\min ^{-1}\right)$ & $\begin{array}{l}\text { Amount } \\
\text { (mg) }\end{array}$ & $\mathrm{k}\left(\min ^{-1}\right)$ & $\begin{array}{c}\text { MB } \\
\text { (ppm) }\end{array}$ & $\mathrm{k}\left(\min ^{-1}\right)$ & $\begin{array}{c}\mathrm{H}_{2} \mathrm{O}_{2} \\
(\mathrm{ml})\end{array}$ & $\mathrm{k}\left(\min ^{-1}\right)$ \\
\hline 5 & 0.0560 & 1.0 & 0.0965 & 20 & 0.2765 & 0.05 & 0.1245 \\
\hline 10 & 0.1256 & 2.0 & 0.1265 & 40 & 0.2015 & 0.10 & 0.1658 \\
\hline 20 & 0.1765 & 5.0 & 0.1725 & 60 & 0.1568 & 0.15 & 0.2158 \\
\hline 25 & 0.2791 & 10.0 & 0.2695 & 80 & 0.9574 & 0.20 & 0.2766 \\
\hline - & - & - & - & 100 & 0.0869 & - & - \\
\hline
\end{tabular}

Table 1. Variation of rate constant with change in reaction parameters.

III. Effect of reaction parameters on rate of reaction:

Reaction parameters such as the amount of catalyst, the concentration of $\mathrm{H}_{2} \mathrm{O}_{2}$, etc., play a vital role in determining the rate of degradation of organic dyes under solar irradiation. In the present study, the effects of such parameters were studied systematically.

a. Concentration of $G O$ :

Due to the large surface area of graphene oxide, a slight change in amount can greatly influence the rate of degradation. The systematic study of the effect of graphene oxide dosage (viz. $5 \%, 10 \%, 20 \%$, and $25 \% \mathrm{w} / \mathrm{w}$ ) on the degradation of methylene blue shows that a larger amount of graphene oxide drives the reaction at a faster rate (Figure 7a). When the reaction was carried out with composites containing 25\% graphene oxide (w/w), the rate constant of the reaction was highest, and the plot for \%degradation vs. Time (min) exhibited an almost linear nature. Thus, $25 \%$ graphene oxide (w/w) was optimized and was used for further experiments.

b. Concentration of Agar@GO Composites:

The degradation of organic dyes is greatly influenced by the amount of catalyst. ${ }^{32}$ To make the process more practical and costeffective, the minimization of catalyst concentration is of utmost importance. The influence of the amount of catalyst (1.0 mg, 2.0 $\mathrm{mg}, 5.0 \mathrm{mg}, 10.0 \mathrm{mg})$ on the degradation of $50.00 \mathrm{ml}$ methylene blue dye $(20 \mathrm{ppm})$ at $\mathrm{H}_{2} \mathrm{O}_{2}-0.20 \mathrm{ml}$ and initial $\mathrm{pH}(\mathrm{pH}=8.4)$ is shown in Figure 7b. $10 \mathrm{mg}$ of composites were found to be more effective in driving the reaction at a faster rate as the rate constant was maximum in this case compared to others. A gradual decrease in rate constant was observed with a decrease in the concentration of composites. All further experiments were carried out at 10.0 mg of Agar@GO composites.

c. Concentration of $\mathrm{H}_{2} \mathrm{O}_{2}$ :

To make the reaction economically feasible, the optimization of $\mathrm{H}_{2} \mathrm{O}_{2}$ is very important. Variation of $\mathrm{H}_{2} \mathrm{O}_{2}$ concentration and its effect on the rate of degradation was monitored, and the data so obtained is shown in Figure 7c. An increase in the rate of reaction was observed with the increase in the concentration of $\mathrm{H}_{2} \mathrm{O}_{2}$. Kinetics studies showed an increase in the rate constant with increasing concentration of $\mathrm{H}_{2} \mathrm{O}_{2}$. The rate was observed to be faster, with $0.20 \mathrm{ml}$ of $\mathrm{H}_{2} \mathrm{O}_{2}$ having a rate constant of 0.2695 $\mathrm{min}^{-1}$. Hence further experiments were carried out at $0.20 \mathrm{ml}$ of $\mathrm{H}_{2} \mathrm{O}_{2}$.

\section{d. Concentration of methylene blue:}

Another parameter that greatly influences the reaction rate is dye concentration. Degradation of methylene blue was studied at various dye concentrations viæ. 20 ppm, 40 ppm, 60 ppm, 80 ppm, and 100 ppm by keeping rest parameters constant (Agar@GO - $10 \mathrm{mg} ; \mathrm{H}_{2} \mathrm{O}_{2}-0.2 \mathrm{ml}$; pH - 8.4). From Figure 7d, it can be seen that, as the concentration of dye increases, the rate of degradation decreases. The rate constant was observed to be highest at a dye concentration of $20 \mathrm{ppm}$. 

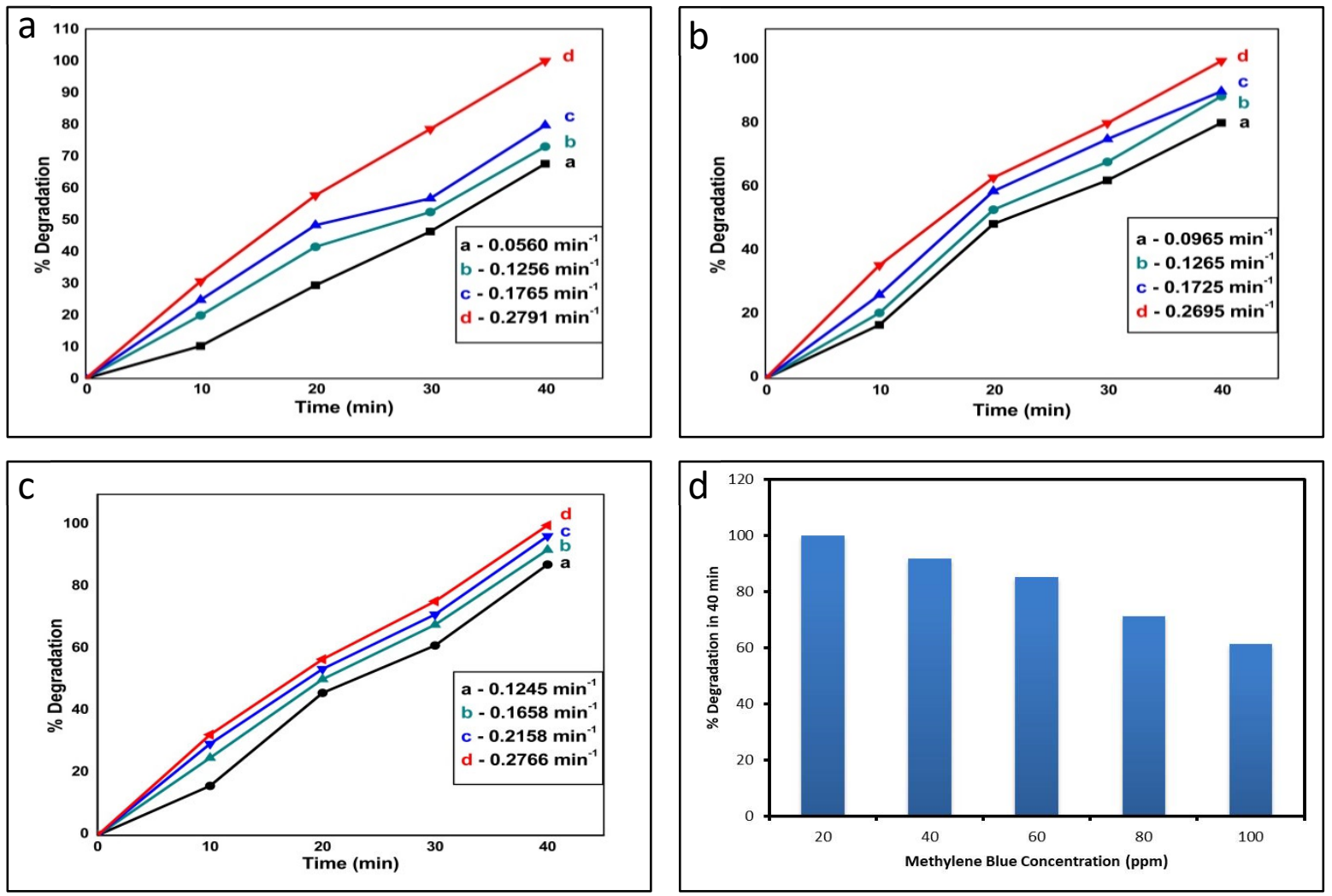

Figure 7. a) Variation of graphene oxide (w/w) with conditions: $\mathrm{a}-5 \%$; $\mathrm{b}-10 \% ; \mathrm{c}-20 \%$; $-25 \% \mathrm{~b}$ ) Variation of Agar@GO with conditions: a - $1.0 \mathrm{mg}$; b $2.0 \mathrm{mg} ; \mathrm{c}-5.0 \mathrm{mg} ; \mathrm{d}-10.0 \mathrm{mg}$ c) Variation of $\mathrm{H}_{2} \mathrm{O}_{2}$ with conditions: a $-0.05 \mathrm{ml}$; $-0.10 \mathrm{ml} ; \mathrm{c}-0.15 \mathrm{ml} ; \mathrm{d}-0.20 \mathrm{ml}$. d) Effect of variation in dye concentration on rate of degradation

\section{Plausible Mechanism of Photo Degradation:}

Agar shows remarkable properties after forming composites with graphene oxide. The lower band gap is one of those remarkable properties which allow these composites to be used for photodegradation of organic dyes. There is no exact mechanism known till date for degradation of organic dyes using Agar@GO composites under solar irradiation. To the best of our knowledge, it is the first report in which the possible mechanism for the degradation of methylene blue is reported using the Agar@GO composites (Figure 8).

When Agar@GO composites are added to the aqueous solution of methylene blue, the molecules of organic dye get adsorbed $\left(\mathrm{MB}^{*}\right)$ on the surface of the agar. Agarose and agaropectin, the two components of agar, contain -OH groups with which the dye molecules interact and get adsorbed. As per the literature survey, methylene blue dye gets adsorbed on agar in the form of monolayers and is irrespective of the concentration of dye. This is due to the presence of a large number of 3, 6-anhydro-Lgalactopyranose units. ${ }^{33}$

$$
\mathrm{MB}+\text { Agar } \rightarrow \mathrm{MB}^{*}+\text { Agar }
$$

\section{Equation 1.}

After irradiating the solution containing Agar@GO composites with visible light, graphene oxide (GO), the key component of Agar@GO composites, releases a pair of holes and electrons. ${ }^{34}$

$\mathrm{GO} \rightarrow \mathrm{e}^{-}+$holes

\section{Equation 2.}

The band gap of graphene oxide $(\sim 3.5 \mathrm{eV})$ allows this generation of pairs on its surface. This photo inductively generated electron-hole pairs then react with the $\mathrm{H}_{2} \mathrm{O}$ and $\mathrm{O}_{2}$ to form active oxygen species.

holes $+\mathrm{H}_{2} \mathrm{O} \rightarrow \mathrm{H}^{+}+\mathrm{OH} \bullet$

$\mathrm{e}^{-}+\mathrm{O}_{2} \rightarrow \mathrm{O}_{2}^{\bullet-}$
Equation 3.

Equation 4. 
$\mathrm{O}_{2} \bullet^{-}+\mathrm{H}^{+} \rightarrow \mathrm{HO}_{2} \bullet$

Equation 5.

The superoxide ion and hydroxyl free radicals generated from the above reaction react with organic dye and forms the degradation products, which are $\mathrm{CO}_{2}$ and $\mathrm{H}_{2} \mathrm{O} .{ }^{35}$

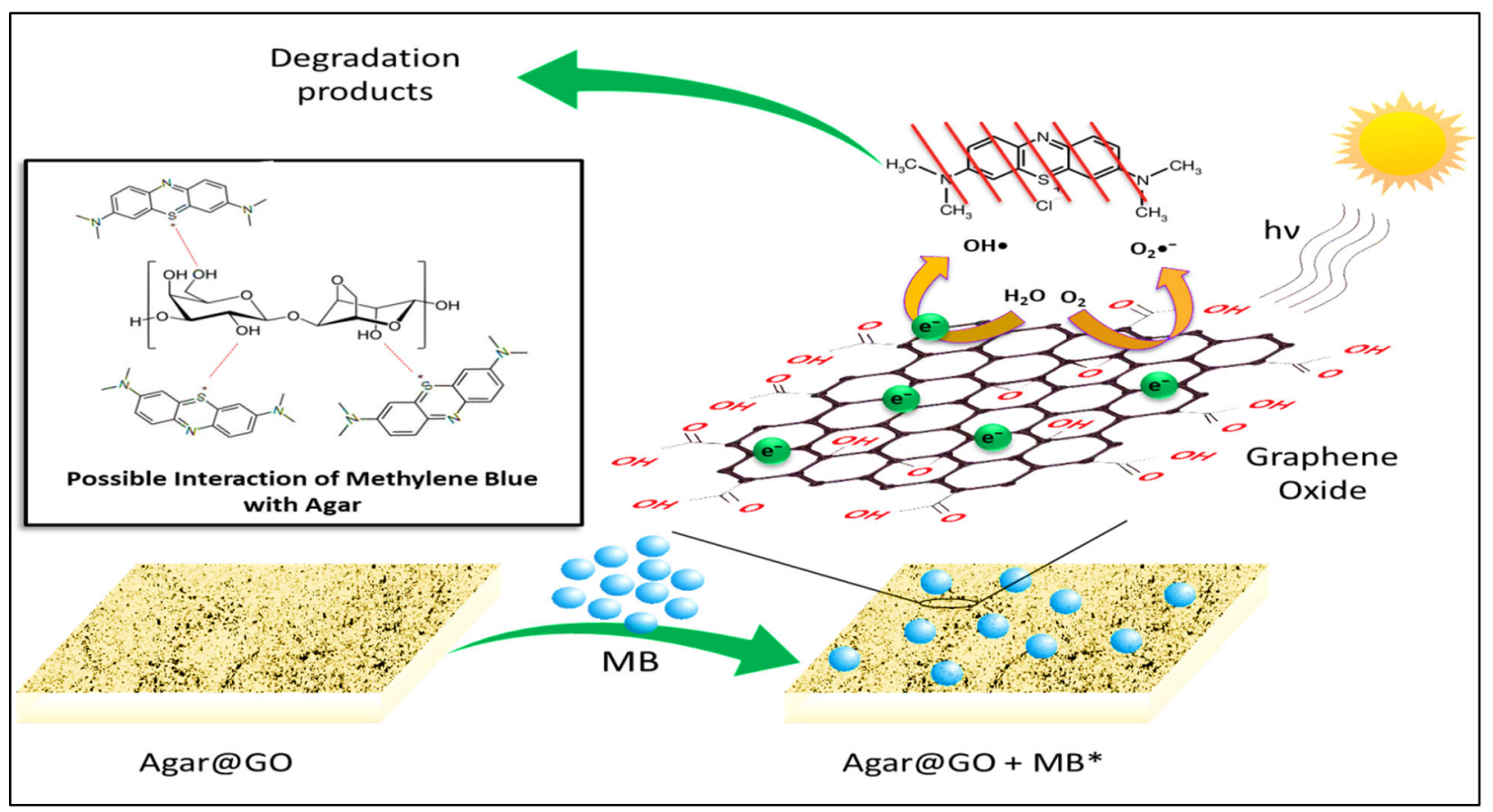

Figure 8. Plausible Mechanism of Photo Degradation of methylene blue dye using Agar@GO composites.

\section{DISCUSSION}

The characteristic peak obtained at around $2 \theta=10^{\circ}$ confirms the formation of graphene oxide. The peak corresponds to the 002 plane and arises due to the increase in interatomic layers, which is associated with the addition of reactive oxygenated groups. The small hump at $2 \theta=43.1^{\circ}$ can be attributed to a turbostratic disorder. ${ }^{36}$ Significant disorder in the as synthesized graphene oxide was shown by the Raman spectra. The two intense peaks, namely D and G, indicate structural disorder in graphene oxide. The D peak, which is generally referred to as disorder band at $1349 \mathrm{~cm}^{-1}$, represents the breathing mode of aromatic rings. ${ }^{37}$ The presence of two additional peaks, namely $2 \mathrm{D}$ and $\mathrm{D}+\mathrm{G}$, also indicated disorderedness in graphene oxide. The results obtained from XRD patterns and Raman spectra for graphene oxide were in excellent agreement with results reported in the literature and thus confirmed its formation. ${ }^{37}$

The analysis of synthesized composites shows structural changes after the incorporation of graphene oxide into the agar. X-ray diffraction patterns of composites reveal that, as the amount of graphene oxide increases, the intensity of the $2 \theta$ peak at $43.13^{\circ}$ increases. A sudden decrease in the $\%$ Transmittance of composites compared to agar is associated with the presence of phenolic groups of graphene oxide. ${ }^{38}$ Agar does not exhibit photo-induced activity, whereas the composites showed active photo nature. It is due to the lower band gap value of $3.58 \mathrm{eV}$ that lies in the photoactive range. All the above observations support the structural changes in the properties of agar are due to the incorporation of graphene oxide.

The UV-visible spectrum recorded for degradation of methylene blue dye shows a decrease in absorbance bands at two wavelengths, $664 \mathrm{~nm}$, and $292 \mathrm{~nm}$, respectively. The decrease in the absorbance band at $664 \mathrm{~nm}$ corresponds to the degradation of chromophores of the dye, whereas a decrease in the band at $292 \mathrm{~nm}$ is attributed to the degradation of organic rings of the dye. ${ }^{39}$ The degradation of organic rings leads to the conversion of harmful dye into less harmful organics such as water and $\mathrm{CO}_{2}$.

The systematic study of influencing factors for rate of reaction shows that the rate of reaction is proportional to the amount of graphene oxide loaded into agar, amount of composites used, and amount of $\mathrm{H}_{2} \mathrm{O}_{2}$, whereas it exhibits inverse relation with the concentration of dye used for degradation. The coefficient of determination factor $\left(\mathrm{R}^{2}\right)$ obtained from the first-order kinetic 
model for an optimized system is $0.994 .^{25}$ The closeness of the $\mathrm{R}^{2}$ value to unity indicates that the data fit the linear model in an excellent agreement and follows pseudo-first-order kinetics.

\section{CONCLUSION}

The synthesized and characterized Agar@GO composites with various weight ratios of graphene oxide showed excellent photocatalytic activity for the degradation of methylene blue dye. All the reactions were carried without 30 minutes of adsorption in the dark and thereby reducing the cost and time of degradation. The relatively high biodegradability and lower band gap could allow the composites to be used as a photo-catalyst for various reactions. Along with excellent photocatalytic activity, the composites could find potential applications in the manufacturing of biocompatible materials like bio-polymers, which could replace polythene based plastic materials.

\section{ACKNOWLEDGEMENTS}

The authors are thankful to Dr. Nilesh Kulkarni from TIFR, Mumbai, for providing XRD data, the Savitribai Phule University of Pune for Raman Spectra. The authors are thankful to Dr. K. G. Bhole and Dr. Suresh Kadam (HOD Physics) K. E. T's, Vinayak Ganesh Vaze College (Autonomous), Mulund, Mumbai, for scientific discussion.

\section{REFERENCES}

1. Lellis, B., Fávaro-Polonio, C.Z., Pamphile, J.A., Polonio, J.C. (2019) Effects of textile dyes on health and the environment and bioremediation potential of living organisms, Biotechnology Research and Innovation 3, 275-290. bttps://doi.org/10.1016/j.biori.2019.09.001.

2. Berradi, M., Hsissou, R., Khudhair, M., Assouag, M., Omar, C., El Bachiri, A., El Harfi, A. (2019) Textile finishing dyes and their impact on aquatic environs, Heliyon 5, e02711. https://doi.org/10.1016/j.heliyon.2019.e02711.

3. Houas, A., Lachheb, H., Ksibi, M., Elaloui, E., Guillard, C., Herrmann, J-M. (2001) Photocatalytic degradation pathway of methylene blue in water, Applied Catalysis B: Environmental 31, 145-157. https:// doi.org/ 10.1016/S0926-3373(00)00276-9.

4. Gadallah, M.A.A. (1996) Phytotoxic effects of industrial and sewage waste waters on growth, chlorophyll content, transpiration rate and relative water content of potted sunflower plants, Water Air Soil Pollut 89, 33-47. https://doi.org/10.1007/BF00300420.

5. Chala, S., Wetchakun, K., Phanichphant, S., Inceesungvorn, B., Wetchakun, N. (2014) Enhanced visible-light-response photocatalytic degradation of methylene blue on Fe-loaded BiVO4 photo-catalyst, Journal of Alloys and Compounds 597, $129-135$. https:// doi.org/10.1016/j.jallcom.2014.01.130.

6. Shannon, M., Bohn, P., Elimelech, M., Georgiadis, J., Mariñas, B., Mayes, A., (2008) Science and technology for water purification in the coming decades, Nature 452, 301-310. https:/ / doi.org/10.1038/ nature06599.

7. Soboleva, N.M., Nosovich, A.A., Goncharuk, V.V. (2007) The heterogenic photocatalysis in water treatment processes, J. Water Chem. Technol. 29, 72-89. bttps://doi.org/10.3103/S1063455X07020038.

8. Pourahmada A., Sohrabnezhad Sh., Kashefian. E. (2010) AgBr/nanoAlMCM-41 visible light photo-catalyst for degradation of methylene blue dye, Spectrochimica Acta Part A 77, 1108-1114. https:// doi.org/10.1016/j.saa.2010.09.006.

9. Rajput, R.S., Pandey, S., Bhadauria, S., (2017) Status of water pollution in relation to industrialization in Rajasthan, Rev Environ Health 32, 245-252. https:// doi.org/10.1515/ reveh-2016-0069.

10. Vidal, O. (2018) 1 - Framework and Challenges, Mineral Resources and Energy, 1-10. https://doi.org/10.1016/B978-1-78548267-0.50001-2.

11. O'Connor, I.A., Golsteijn, L., Hendriks A.J. (2016) Review of the partitioning of chemicals into different plastics: Consequences for the risk assessment of marine plastic debris, Marine Pollution Bulletin 113, 17-24. bttps://doi.org/10.1016/j.marpolbul.2016.07.021.

12. Liu J., Wang Z., Liu L., Chen W. (2011) Reduced graphene oxide as capturer of dyes and electrons during photocatalysis: surface wrapping and capture promoted efficiency, Phys Chem Chem Phys 29, 13216-13221. bttps://doi.org/10.1039/c1cp20504h.

13. Liu, T., Li, Y., Du, Q., Sun, J., Jiao, Y., Yang, G., Wang, Z., Xia, Y., Zhang, W., Wang, K., Zhu, H., Wu, D. (2012) Adsorption of methylene blue from aqueous solution by graphene, Colloids and Surfaces B: Biointerfaces 90, 197-203. bttps:// doi.org/10.1016/j.colsurfb.2011.10.019.

14. Liu, Y., Liu, C., Liu, Y., (2011) Investigation on fluorescence quenching of dyes by graphite oxide and graphene, Applied Surface Science 257, 5513-5518. https:// doi.org/ 10.1016/j.apsusc.2010.12.136.

15. Ramesha, G.K., Kumara, A.V., Muralidhara, H.B., Sampath, S. (2011) Graphene and graphene oxide as effective adsorbents toward anionic and cationic dyes, J Colloid Interface Sci 361, 270-277. https://doi.org/10.1016/j.jcis.2011.05.050.

16. Smith, A.T., LaChance, A.M., Zeng, S., Liu, B., Sun, L. (2019) Synthesis, properties, and applications of graphene oxide/reduced graphene oxide and their nanocomposites, Nano Materials Science 1, 31-47. bttps:// doi.org/10.1016/j.nanoms.2019.02.004.

17. Paredes, J. I., Villar-Rodil, S., Martínez-Alonso, A., Tascón, J. M. D. (2008) Graphene Oxide Dispersions in Organic Solvents, Langmuir 24, 10560-10564. https:// doi.org/10.1021/la801744a. 
18. Chen, S., Zhu, J., Wu, X., Han, Q., Wang, X. (2010) Graphene oxide-MnO2 nanocomposites for supercapacitors, ACS Nano 4, 2822-2830. bttps://doi.org/10.1021/nn901311t.

19. Li, Z.J., Yang, B.C., Zhang, S.R., Zhao, C.M. (2012) Graphene oxide with improved electrical conductivity for super capacitor electrodes, Applied Surface Science 258, 3726-3731. bttps:// doi.org/10.1016/j.apsusc.2011.12.015.

20. Piao, Y., Chen, B. (2016) One-pot synthesis and characterization of reduced graphene oxide-gelatin nanocomposite hydrogels, RSC Adv 6, 6171-6181. https://doi.org/10.1039/C5RA20674J.

21. Freile-Pelegrín, Y., Madera-Santana, T., Robledo, D., Veleva, L., Quintana, P., Azamar, J.A. (2007) Degradation of agar films in a humid tropical climate: Thermal, mechanical, morphological and structural changes, Polymer Degradation and Stability 92 , 244-252. https://doi.org/10.1016/j.polymdegradstab.2006.11.005.

22. Mesbah, N.M., Wiegel, J., (2006) 19 Isolation, Cultivation and Characterization of Alkalithermophiles, Methods in Microbiology 35, 451-468. https:/ / doi.org/10.1016/S0580-9517(08)70022-9.

23. Azim, M.B., Tanima, I.A., Rezaula, R.M., Tareqa, R., Rahula, A.H., Kurnyb A.S.W., Gulshanb, F. (2018) Degradation of methylene blue using graphene oxide-tin oxide nanocomposite as photo-catalyst, arXiv: Materials Science, 202-209, Paper ID: C117.

24. Seema, H., Kemp, K.C., Chandra, V., Kim, K.S. (2012) Graphene-SnO2 composites for highly efficient photocatalytic degradation of methylene blue under sunlight, Nanotechnology 23, 355705. https:// doi.org/10.1088/0957-4484/23/35/355705.

25. Chen, L., Li, Y., Du, Q., Wang, Z., Xia, Y., Yedinak, E., Lou, J., Ci, L. (2017) High performance agar/graphene oxide composite aerogel for methylene blue removal, Carbohydrate Polymers 155, 345-353. bttps://doi.org/10.1016/j.carbpol.2016.08.047.

26. Ionita, M., Crica, L.E., Tiainen, H., Haugen, H.J., Vasile, E., Dinescu, S., Costache, M., Iovu, H. (2015) Gelatin-Poly (vinyl alcohol) Porous Biocomposites Reinforced with Graphene Oxide as Biomaterials, J. Mater, Chem. B 4, $282-291$. bttps:// doi.org/10.1039/C5TB02132D.

27. Belay, M., Nagarale, R.K., Verma, V. (2017) Preparation and characterization of graphene-agar and graphene oxide-agar composites, Journal of Applied Polymer Science 134, 45085. https:/ / doi.org/10.1002/ APP.45085.

28. Hummers, W. S.; Offeman, R. E. (1958) Preparation of Graphitic Oxide, J. Am. Chem. Soc. 80, 1339-1339. https://doi.org/10.1021/ja01539a017.

29. Gascho, J.L.S., Costa, S.F., Recco, A.A.C., Pezzin, S.H. (2019) Graphene Oxide Films Obtained by Vacuum Filtration: X-Ray Diffraction Evidence of Crystalline Reorganization, Journal of Nanomaterials 2019, 1-12. https:// doi.org/10.1155/2019/5963148.

30. Kanmani, P., Rhim, J.W. (2014) Antimicrobial and physical-mechanical properties of agar-based films incorporated with grapefruit seed extract, Carbohydrate Polymers 102, 708-716. https:// doi.org/10.1016/j.carbpol.2013.10.099.

31. Debanath, M.K., Karmakar, S. (2013) Study of blueshift of optical band gap in zinc oxide (ZnO) nanoparticles prepared by low-temperature wet chemical method, Materials Letters 111, 116-119. bttps:/ / doi.org/10.1016/j.matlet.2013.08.069.

32. Jangam, K., Patil, K., Balgude, S., Patange, S., More, P. (2021) Synthesis and characterization of magnetically separable Zn1xCoxFeMnO4 nanoferrites as highly efficient photo-catalyst for degradation of dye under solar light irradiation, Journal of Physics and Chemistry of Solids 148, 109700. https:/ / doi.org/10.1016/j.jpcs.2020.109700.

33. Samiey, B., Ashoori, F. (2012) Adsorptive removal of methylene blue by agar: effects of $\mathrm{NaCl}$ and ethanol, Chemistry Central Journal 06, 14. https:/ / doi.org/ 10.1186/1752-153X-6-14.

34. Krishnamoorthy, K., Mohan, R., Kim, S.-J. (2011) Graphene oxide as a photocatalytic material, Applied physics letters 98 , 244101. bttps://doi.org/10.1063/1.3599453.

35. Jangam, K., Patil, K., Balgude, S., Patange, S., More, P. (2020) Magnetically separable Zn1-xCo0.5xMg0.5xFe2O4 ferrites: stable and efficient sunlight-driven photocatalyst for environmental remediation, RSC Adv. 10, 42766-42776. bttps:/ / doi.org/ 10.1039/DORA08172H.

36. Emiru, T.F., Ayele D.W. (2017) Controlled synthesis, characterization and reduction of graphene oxide: A convenient method for large scale production, Egyptian Journal of Basic and Applied Sciences 04, 74-79. bttps:/ / doi.org/10.1016/j.ejbas.2016.11.002.

37. Shahriary, L., Athawale, A. (2014) Graphene Oxide Synthesized by using Modified Hummers Approach, International Journal of Renewable Energy and Environmental Engineering 02, 58-63.

38. Ganguly, A., Sharma, S., Papakonstantinou, P., Hamilton, J. (2011) Probing the thermal deoxygenation of graphene oxide using high-resolution in situ X-ray-Based spectroscopies, J. Phys. Chem. C 115, 17009-17019. https://doi.org/10.1021/jp203741y.

39. Rauf, M.A., Meetani, M.A., Khaleel, A., Ahmed, A. (2010) Photocatalytic degradation of Methylene Blue using a mixed catalyst and product analysis by LC/MS, Chemical Engineering Journal 157, 373-378. https:// doi.org/10.1016/j.cej.2009.11.017.

\section{ABOUT STUDENT AUTHORS}

Shreyas Dindorkar is an undergraduate student at K. E. T.'s V. G. Vaze College (Autonomous), Mulund (E) majoring in chemistry. After completing his Bachelor's degree in April 2021, his objective is to seek an R\&D chemist position in quantum materials and theoretical chemistry.

Jaymin Mistry is an undergraduate student at K. E. T.'s V. G. Vaze College (Autonomous), Mulund (E). He will graduate in April 2021 with a Bachelor's degree. He aims to pursue higher studies in data science. 
Jayesh Hire is an undergraduate student at K. E. T.'s V. G. Vaze College (Autonomous), Mulund (E). He will complete his Bachelor's degree in April 2021 with physics as majors and aims to become an innovative researcher in the field of optics.

Khushi Jain is an undergraduate Biotechnology student at K. E. T.'s V. G. Vaze College (Autonomous), Mulund (E). She will graduate with a B.S. degree in April 2021 with an objective of high-quality research in the field of cancer biotechnology and environmental wellbeing through an interdisciplinary approach.

Nandini Khona is an undergraduate Biotechnology student at K. E. T.'s V. G. Vaze College (Autonomous), Mulund (E). After getting a B.S. degree in April 2021, she plans to pursue higher studies in the field of biotechnology.

Shreya Peddakolmi is an undergraduate Biotechnology student at K. E. T.'s V. G. Vaze College (Autonomous), Mulund (E), and will complete her B.S. degree in April 2021. She is planning for higher studies and research in the field of animal biotechnology.

\section{PRESS SUMMARY}

Colorants used in textile industries are organic dyes that affect the quality of water and have been recognized as the root of pollution over the last few years. The current study presents the investigation of structural and optical properties of biocompatible Agar@GO composites and their photocatalytic activity in the degradation of organic dyes. The synthesis was carried out by mixing the agar solution and dispersion of graphene oxide in the desired ratio. The critically analyzed results show that the reaction follows the pseudo first order. Only $10.0 \mathrm{mg}$ of synthesized composites were found to show an excellent activity for degradation of methylene blue dye and thus increasing the economic viability of the reaction. Within a short period of 40 minutes, $100 \%$ degradation was observed. 


\title{
The Evolution of Multidrug Resistance in an Isolated Pseudomonas Strain
}

\author{
Allison Grodnick*a, Ashley Fink, , Timothy J. Johnson ${ }^{b c}$, \& David Mitchelk \\ ${ }^{a}$ Department of Biology, College of St. Benedict St. John's University, Collegeville, $M N$ \\ ${ }^{b}$ Department of Veterinary and Biomedical Sciences, University of Minnesota, St. Paul, MN \\ 'Mid-Central Research and Outreach Center, University of Minnesota, Willmar, MN \\ https:// doi.org/10.33697/ ajur.2020.026 \\ Student:aegrodnick@gmail.com* \\ Mentor:dmitchell@csbsju.edu
}

\begin{abstract}
As an unintentional result of the extensive use of antibiotics in healthcare and agriculture, antibiotics have become an increasingly prevalent selective pressure on bacteria. This forces bacteria to evolve and acquire antibiotic-resistant genes or mutations in order to survive. Suppose a bacterial strain acquires resistance to three or more antibiotics. In that case, it is deemed multidrug-resistant (MDR), and it becomes a potentially more serious problem to solve in the context of healthcare. This study aims to evaluate the acquisition of resistance to multiple antibiotic drugs by an initially susceptible isolated bacterium from a Minnesota forest environment. The bacterium was found to be Pseudomonas by 16 s rRNA gene sequencing. Three antibiotics, neomycin, ciprofloxacin, and imipenem, each from a different drug class, were selected to see if this isolate could become resistant over time and exposure. The bacterial strain developed resistance to the selected antibiotics through a series of sequential exposures to increasing concentrations of each drug in this order. As determined by a disc susceptibility test, the initial isolate acquired resistance to all three selected antibiotics. Single nucleotide polymorphisms (SNPs) between the original isolate and the final resistant strain were identified. These SNPs suggest that mutations to efflux transporters and antibiotic protein targets play a role in acquiring and maintaining antibiotic resistance.
\end{abstract}

\section{KEYWORDS}

Multidrug Resistance; Antibiotics; Neomycin; Ciprofloxacin; Imipenem; Pseudomonas; Evolution; MDR; Minnesota Environment

\section{INTRODUCTION}

Like all species, bacteria evolve. Depending on the environmental factors and stresses, bacteria evolve in order to survive. These factors can range from a change in the environment to human influence. Within the realm of human influence lies antibiotics. While antibiotics are a powerful tool in medicine, they also present a new environmental stressor to bacteria. ${ }^{1}$ As an unintentional result of healthcare and agriculture uses, antibiotics have become an increasingly prevalent selective pressure. ${ }^{2}$ This stressor promotes a bacterium's evolution to become resistant to the antibiotic it is exposed to survive. Those that evolve are then capable of out-competing susceptible bacterial strains, so the resistant strain reproduces more quickly and in greater quantity. When this occurs, it can be difficult to treat patients infected with the now resistant bacteria. This has become a rising health issue as bacteria continue to develop antibiotic resistance; they were previously susceptible. Drug resistance has arisen in some capacity for almost all antibiotic drugs on the market, and certain pathogenic bacterial strains have been characterized as multidrug-resistant organisms. ${ }^{3}$

Antibiotics play an essential role in modern medicine. The average life expectancy before the discovery of antibiotics was 47 years old. ${ }^{4}$ This average life expectancy rose to 78 years following 35 years of antibiotic discovery due to the newly found ability to treat a multitude of infectious diseases. ${ }^{4}$ This resulted in drastic public health changes that improved both quality and length of life. While antibiotics are an essential tool in medicine, problems have begun to arise due to the overuse of antibiotics, leading to antibiotic resistance in bacteria and other microorganisms. The World Health Organization has deemed antibiotic resistance a major public health threat. ${ }^{5}$ The CDC reported antibiotic-resistant bacteria and fungi to have caused at least 2.8 million infections in 2019 in the United States. ${ }^{6}$ Of these cases, 35,000 led to death. Not only does antibiotic resistance affect public health, but it has a negative economic impact too. In the United States, antibiotic-resistant infections are estimated to cost 20 billion dollars per year. ${ }^{5}$ Due to both the health and economic impacts of drug resistance, this problem must be addressed.

Antibiotic drugs fall into four different classes based on their mechanism of action: cell wall inhibitors, protein synthesis inhibitors, DNA and RNA synthesis inhibitors, and metabolic pathway inhibitors. ${ }^{2}$ Not only can bacteria evolve resistance to one 
antibiotic or a class of antibiotics, but they are capable of evolving resistance to multiple drugs and multiple drug classes. The acquisition of resistance to antibiotics holds more serious consequences as a bacterium develops resistance to multiple classes of antibiotics. The term multidrug resistance (MDR) is given to a bacterial strain resistant to a minimum of three different classes of antibiotics. ${ }^{7}$ Bacteria are resistant to antibiotics, either intrinsically or through acquired mutations. ${ }^{7}$ Common mechanisms of resistance include efflux pumps, changes in cell wall permeability, modification of an antibiotic by enzymatic action, antibiotic degradation, use of alternative metabolic pathways, modification of the antibiotic target, and overexpression of the enzyme targeted by an antibiotic. ${ }^{8}$ Efflux pumps have been found to play a pivotal role in both intrinsic and adaptive resistance. Efflux pumps are components of bacterial membranes, so bacteria naturally express these pumps. ${ }^{9}$ In the presence of antibiotic selective pressure, the overexpression of efflux pumps as well as mutations in these pumps have been observed, which has led to efflux pumps becoming a point of interest in the study of antibiotic resistance. ${ }^{9}$

In this study, a bacterium was isolated from the St. John's University arboretum; 16s rRNA gene sequencing determined it to be a Psendomonas. Pseudomonas are known to innately express various efflux pumps playing a role in their intrinsic antibiotic-resistant abilities. ${ }^{9}$ The isolate was intrinsically susceptible to an array of antibiotics by antibiotic susceptibility disc tests in which susceptibility is measured by the zone of inhibition created. Three of these antibiotics were selected to evaluate the isolate's ability to acquire MDR: neomycin, ciprofloxacin, and imipenem.

This isolate set the stage for this study's experiment to see if antibiotic resistance could be evolved in the laboratory via repeated exposures to a particular antibiotic. Once resistance was demonstrated via an antibiotic disc test that previously killed the isolate, the isolate's genome was sequenced to look for possible SNPs that arose that may play a role in the acquisition of resistance. The goal was to gain insight into the acquisition of antibiotic resistance in an isolate from an environmental setting rather than with a known pathogenic bacterial strain as other studies have demonstrated.4,7

There are a variety of commonly mutated proteins in resistant Pseudomonas species. ${ }^{4}$ Depending on the selective pressure of a given antibiotic, different mutations may arise in order for the strain to become resistant since antibiotics are bactericidal through a variety of mechanisms.

Neomycin is an aminoglycoside antibiotic. ${ }^{8}$ It is bactericidal through its inhibition of protein synthesis. ${ }^{8}$ Its mechanism of action lies in binding to the 30 s subunit that is part of the bacterial ribosome. ${ }^{10}$ Due to this binding action, the ribosome cannot properly translate mRNA into protein, which is detrimental to the cell..$^{10}$ In order to survive, bacteria have developed mechanisms of resistance to this class of antibiotics. ${ }^{8}$ A variety of mechanisms have been identified in the gaining of resistance to aminoglycosides: active efflux, permeability changes, ribosome modifications, and degradation of aminoglycosides by enzymatic activity. ${ }^{8}$ These changes are attributed to a variety of genetic mutations, so resistance to this antibiotic originates from a variety of genes. ${ }^{8}$

Ciprofloxacin belongs to the DNA synthesis inhibitor drug class. It is a fluoroquinolone. ${ }^{8}$ It works by inhibiting DNA gyrase and topoisomerase IV. ${ }^{8}$ Without these enzymes, the bacterium cannot replicate its DNA, which is an essential part of the growth of a bacterial population. ${ }^{8}$ In order to gain resistance, a bacterium typically gains a random mutation in one of the genes that codes for these targeted enzymes. ${ }^{8}$ A random mutation in a targeted enzyme may then alter the enzyme's structure, which may lead to altered enzyme function. ${ }^{8}$ Resistant bacteria have also been observed to decrease membrane permeability and perform active efflux in order to gain resistance to this class of drugs. ${ }^{8}$

Imipenem falls into the cell wall inhibitor drug class. It is a carbapenem that belongs to a group of antibiotics called $\beta$-lactams. ${ }^{8}$ These antibiotics work by weakening the cell wall of a bacterium and making it more susceptible to lysing due to osmotic pressure. ${ }^{8}$ It works by binding penicillin-binding proteins, which play a role in the cross-linking of peptidoglycan chains, which are structurally needed in the cell wall. ${ }^{8}$ Many bacteria have successfully acquired resistance to drugs in this class through the expression of beta-lactamase, which destroys the beta-lactam ring in these drugs. ${ }^{8} \mathrm{~A}$ variety of other mechanisms to resistance have been observed, but beta-lactamase production is the most common. ${ }^{8}$

Taken together, these selected antibiotics target bacteria cells using very different and distinct mechanisms. The isolated Pseudomonas was evaluated for its ability to gain and maintain resistance to each of these antibiotics. This study aims to provide insight into this acquisition and maintenance of multidrug resistance in a bacterial strain isolated from an environmental setting.

\section{METHODS AND PROCEDURES}

\section{Materials}

Tryptic soy agar (TSA) and tryptic soy broth (TSB) were used for growing bacteria on plates and in liquid cultures, respectively

(BD). The TSB flasks were incubated at $25^{\circ} \mathrm{C}$ and $200 \mathrm{rpm}$ (New Brunswick Scientific C25 Incubator Shaker) for 24 to 72 hours. 
Optical density at $600 \mathrm{~nm}$ was taken to measure culture density (OD $\mathrm{OD}_{60}$; Beckman DU640 spectrophotometer). Neomycin trisulfate salt hydrate (Sigma Aldrich, CAS 1405-10-3), ciprofloxacin (Sigma Aldrich, CAS 85721-33-1), and imipenem monohydrate (Sigma Aldrich, CAS 74431-23-5) powders were used for the series of sequential exposures. $30 \mu \mathrm{g} / \mathrm{mL}$ neomycin discs (BD BBL, 231313), $5 \mu \mathrm{g} / \mathrm{mL}$ ciprofloxacin discs (BD BBL, 231658), and $10 \mu \mathrm{g} / \mathrm{mL}$ imipenem discs (BD BBL, 231645) were used in the antibiotic susceptibility disc tests.

\section{Colony isolation and $r \mathrm{RN} A$ gene sequencing identification}

Water samples from a pond located in the St. John's University Arboretum in Collegeville, MN, were collected and streaked out on a TSA plate. A single colony was selected and isolated by line streaking until pure. A pure culture of the isolated bacteria was subject to PCR amplification using universal primers U341F and UA1406R that recognize an 1100 bp segment of the 16s rRNA gene. PCR products were run on a 1.5\% agarose gel and confirmed to be $1100 \mathrm{bp}$ in length. PCR products were then purified (QIAquick PCR Purification Kit, QIAGEN), pre-mixed with the forward universal primer U341F, and sent to GeneWiz (South Plainfield, NJ) for sequencing. FASTA files were then used in a BLAST and the ARB-SLIVA project aligner to identify each organism's most likely genus and species.

\section{Antibiotic exposure}

The isolate was determined to be susceptible to neomycin $(30 \mu \mathrm{g} / \mathrm{mL})$, ciprofloxacin $(5 \mu \mathrm{g} / \mathrm{mL})$, and imipenem $(10 \mu \mathrm{g} / \mathrm{mL})$ by an antibiotic susceptibility disc test. The zone of inhibition (measured in millimeters) created by the disc was used as a means of measuring susceptibility to the given antibiotic.

A flask containing $50 \mathrm{~mL}$ of TSB with a concentration of $5 \mu \mathrm{g} / \mathrm{mL}$ of neomycin was inoculated with the original Pseudomonas isolate at time $0 \mathrm{hr}$. The optical density (OD600) was measured every two hours until an absorbance of 1.0 was reached after 24 to 72 hours. Once achieved, the evolved bacteria were streaked out onto a TSA plate without the antibiotic present. This process was repeated with the evolved bacterial strain at a higher concentration of neomycin until the strain acquired resistance to neomycin at a concentration of $30 \mu \mathrm{g} / \mathrm{mL}$. The bacterial strain developed resistance to neomycin at the increments of $5 \mu \mathrm{g} / \mathrm{mL}$, $7.5 \mu \mathrm{g} / \mathrm{mL}, 15 \mu \mathrm{g} / \mathrm{mL}, 20 \mu \mathrm{g} / \mathrm{mL}, 25 \mu \mathrm{g} / \mathrm{mL}$, and $30 \mu \mathrm{g} / \mathrm{mL}$ in sequential order. There were a total of six transfers within this series in which the isolate was streaked for isolation before each transfer. To confirm the acquisition of resistance, the bacterial strain at each neomycin concentration increment was plated on a TSA plate and tested against the $30 \mu \mathrm{g} / \mathrm{mL}$ neomycin disc. Zones of inhibition were measured after a 48-hour growth period.

Once the acquisition of antibiotic resistance to neomycin at the target concentration of $30 \mu \mathrm{g} / \mathrm{mL}$ was confirmed by the disc test, the resistant strain underwent a process of sequential exposure again but with ciprofloxacin. The strain developed resistance to ciprofloxacin at the increments $2.5 \mu \mathrm{g} / \mathrm{mL}, 3.5 \mu \mathrm{g} / \mathrm{mL}, 4.5 \mu \mathrm{g} / \mathrm{mL}$, and $5 \mu \mathrm{g} / \mathrm{mL}$. There were a total of four transfers within this series, and colonies were streaked for isolation before each new transfer. In order to confirm the acquisition of resistance, the bacterial strain at each ciprofloxacin concentration increment was plated on a TSA plate and tested against the $5 \mu \mathrm{g} / \mathrm{mL}$ ciprofloxacin disc. Zones of inhibition were measured after a 48-hour growth period.

This process of sequential exposure was repeated once more with imipenem. The initial bacterial strain used for this round of sequential exposure was the strain previously made resistant to $30 \mu \mathrm{g} / \mathrm{mL}$ neomycin and $5 \mu \mathrm{g} / \mathrm{mL}$ ciprofloxacin. The bacterial strain developed resistance to imipenem at the increments $1 \mu \mathrm{g} / \mathrm{mL}, 3 \mu \mathrm{g} / \mathrm{mL}, 5 \mu \mathrm{g} / \mathrm{mL}, 7.5 \mu \mathrm{g} / \mathrm{mL}$, and $10 \mu \mathrm{g} / \mathrm{mL}$. There were a total of five transfers in this series, and each isolate was streaked for isolation before a new transfer. Following the acquisition of antibiotic resistance to imipenem at the target concentration of $10 \mu \mathrm{g} / \mathrm{mL}$, the bacterial strain at each imipenem concentration increment was plated on a TSA plate and tested against the $10 \mu \mathrm{g} / \mathrm{mL}$ ciprofloxacin disc to confirm that resistance to imipenem was acquired. Zones of inhibition were measured after a 48-hour growth period.

Throughout this series of sequential exposures, there were a total of 15 transfers of the bacterial strain to an increased antibiotic concentration. In the neomycin experiment set, there were six transfers. In the ciprofloxacin experiment set, there were five transfers. Lastly, in the imipenem experiment set, there were four transfers.

\section{Antibiotic susceptibility disc test for $M D R$}

TSA plates containing $30 \mu \mathrm{g} / \mathrm{mL}$ neomycin and $10 \mu \mathrm{g} / \mathrm{mL}$ imipenem were made. The bacterial strain at each major point in the study was plated onto these antibiotic TSA plates, and a $5 \mu \mathrm{g} / \mathrm{mL}$ ciprofloxacin disc was used. The strains used were the initial isolate, the $30 \mu \mathrm{g} / \mathrm{mL}$ neomycin resistant strain, the $30 \mu \mathrm{g} / \mathrm{mL}$ neomycin and $5 \mu \mathrm{g} / \mathrm{mL}$ ciprofloxacin-resistant strain, and the 30 $\mu \mathrm{g} / \mathrm{mL}$ neomycin, $5 \mu \mathrm{g} / \mathrm{mL}$ ciprofloxacin, and $10 \mu \mathrm{g} / \mathrm{mL}$ imipenem resistant strain. Zones of inhibition were measured after a 
48-hour growth period. Refer to Table 1 for the standard zone of inhibition measurements used to determine whether or not a strain was resistant.

Genome Sequencing

The original isolate's genome, the neomycin resistant strain, the neomycin, and ciprofloxacin-resistant strain, and the neomycin, ciprofloxacin, and imipenem resistant strain were sequenced and analyzed by Illumina sequencing. Flask growths were grown overnight on MacConkey agar to check for visible contamination, then a streak of colonies from the plate was re-grown overnight in $2 \mathrm{~mL} \mathrm{LB}$ broth with shaking at $37^{\circ} \mathrm{C}$. DNA was extracted from overnight growths of each strain using the Qiagen DNEasy Kit. Library preparations were performed with Illumina reagents (Nextera XT DNA library kit). DNA sequencing was performed on a MiSeq machine at the University of Minnesota Genomics Center (Saint Paul, MN, USA). A range of 81,420-666,386 highquality reads (Q30 average) was generated for each strain, corresponding to approximately 10-30-fold coverage for each strain sequenced. Following quality filtering, all genomes were assembled using SPAdes. ${ }^{11}$ The original strain's assembly was used as a reference for read mapping to identify SNPs, with 463 ( $\mathrm{min}=120)$ contigs totaling 6,743,307 bp. The assembly of this genome was annotated using Prokka. ${ }^{12}$ Reads from the original strain and evolved strains were mapped to the original strain assembly using snippy (https://github.com/tseemann/snippy), with a similarity fraction of 0.9 and minimum coverage of $8 \mathrm{x}$ for variant calling. Variants were called at high minimum frequency $(>90 \%)$ to identify true variants relative to the reference genome and other sequenced strains in this study. Mutations unique to each strain sequenced, relative to the parent strain sequenced, were identified and annotated. MAUVE. ${ }^{13}$ was used to align the original and evolved sequences for the assessment of any large-scale genomic changes.

\section{RESULTS AND DISCUSSION}

Antibiotic Exposure and Disc Test

The basis of the experiment is to evaluate the ability of a bacterial strain to gain antibiotic resistance. The original bacterial isolate is referred to as strain 1.

The optical density at $600 \mathrm{~nm}$ was used to measure the growth of the bacteria. Each flask was grown to a minimum optical density of 1.0. Due to overnight growth in some of the flasks, there were cases where flasks exceeded the 1.0 minimum optical density. A growth curve was made with the average of two replicate flasks' optical densities at each time interval measured for each antibiotic concentration. Additionally, the standard deviation was calculated and applied as error bars. The majority of the standard deviations calculated were small, which is evident from the size of the error bars. The growth curve for the bacterial strain at each neomycin concentration is depicted in Figure 1; the neomycin resistant strain is referred to as "strain 2." Figure 2 depicts the growth curve for the bacterial strain at each ciprofloxacin concentration; this neomycin and ciprofloxacin-resistant strain is referred to as "strain 3." Lastly, Figure 3 depicts the growth curve of the bacterial strain at each imipenem concentration; this final strain is referred to as "strain 4." For clarity, it is worth noting that the original isolated bacterial strain, parental strain, is referred to as "strain 1. ."
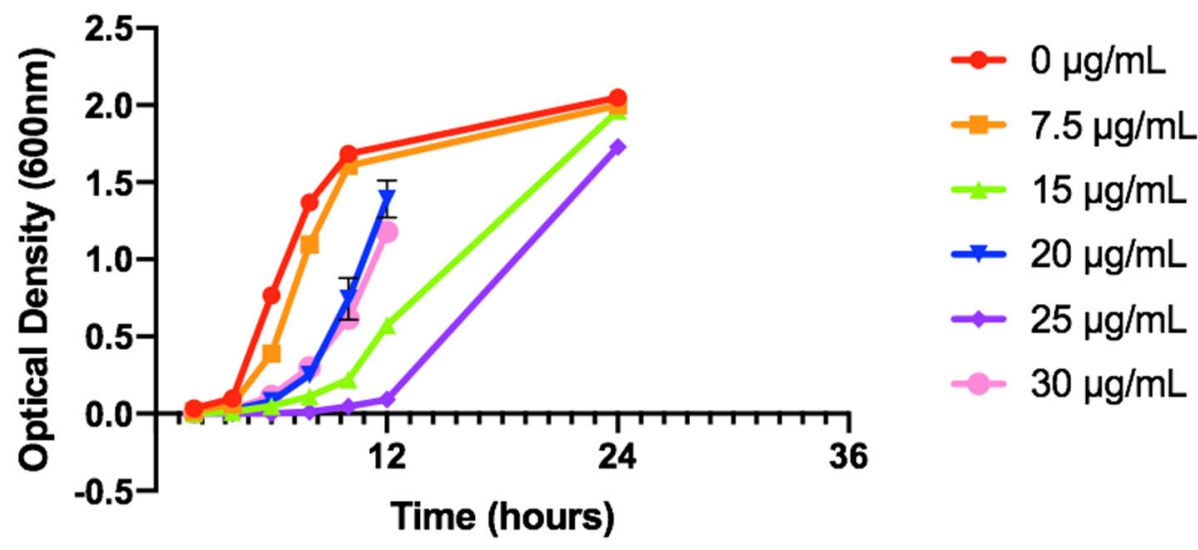

Figure 1. Growth curve for the series sequential exposures to neomycin at each concentration. 


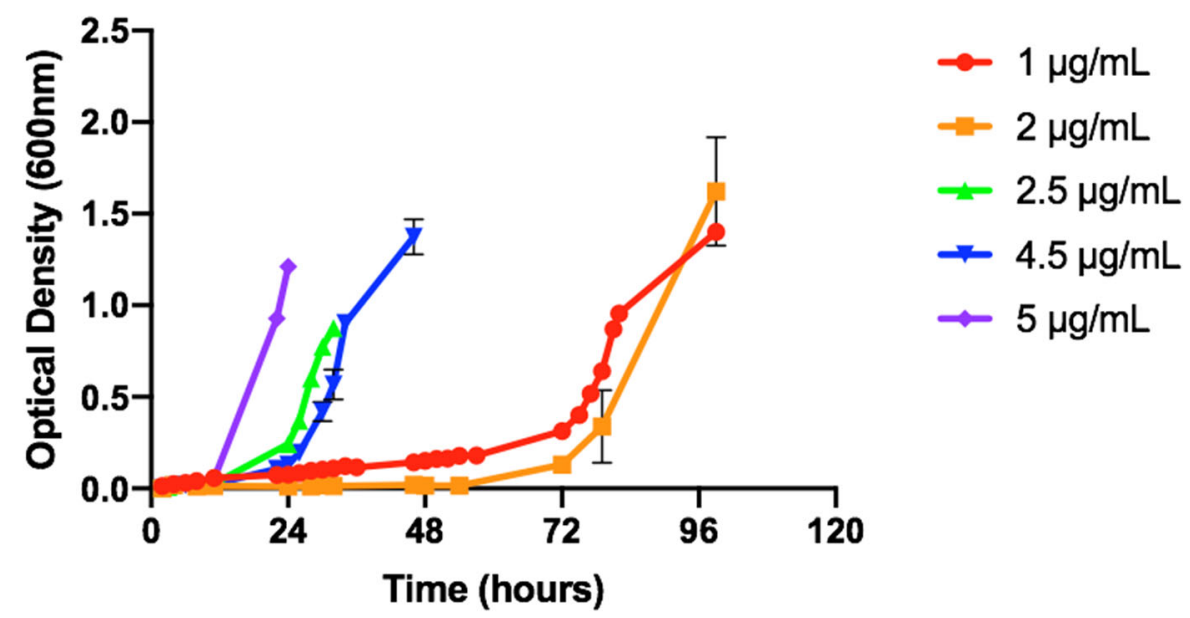

Figure 2. Growth curve for the series sequential exposures to ciprofloxacin at each concentration.

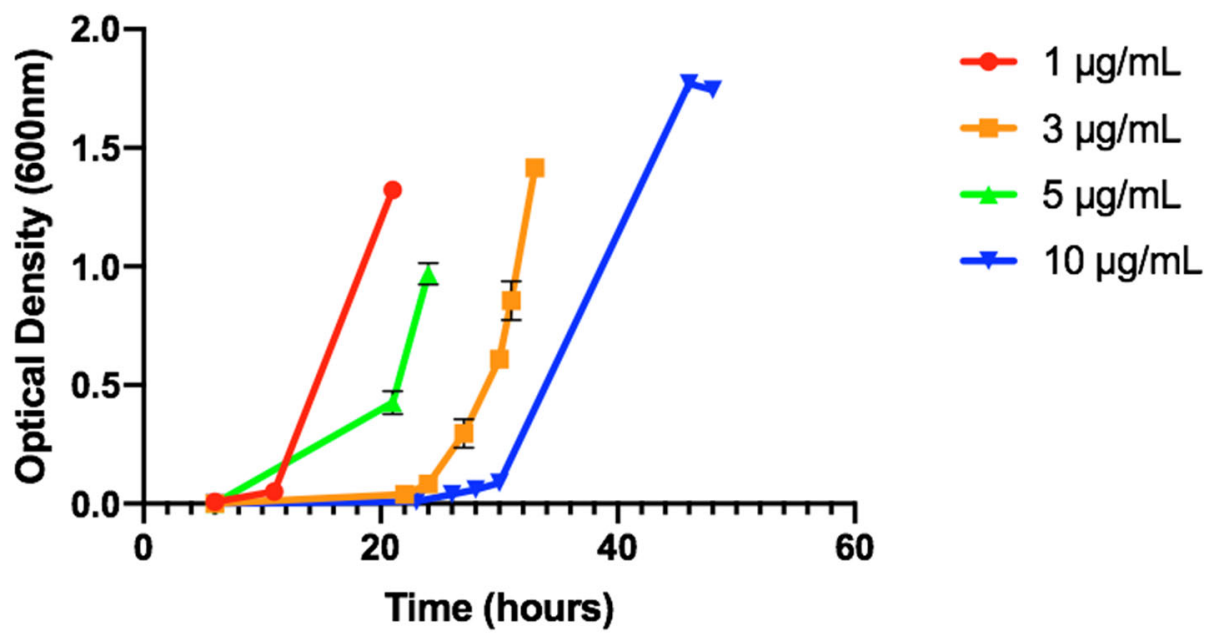

Figure 3. Growth curve for the series sequential exposures to imipenem at each concentration

\begin{tabular}{|l|l|l|l|}
\hline Antibiotic & Resistant & Intermediate & Susceptible \\
\hline Neomycin & $12 \geq$ & $13-16$ & $\geq 17$ \\
\hline Ciprofloxacin & $15 \geq$ & $16-20$ & $\geq 21$ \\
\hline Imipenem & $13 \geq$ & $14-15$ & $\geq 16$ \\
\hline
\end{tabular}

Table 1. Standard zones of inhibition ( $\mathrm{mm}$ ) for each antibiotic according to Becton Dickinson BBL Sensi-Disc Antimicrobial Sensibility Test standards.

\begin{tabular}{|l|l|}
\hline Antibiotic & Initial Zone of Inhibition \\
\hline Neomycin & 20 \\
\hline Ciprofloxacin & Not Recorded \\
\hline Imipenem & 39 \\
\hline
\end{tabular}

Table 2. Initial zones of inhibition (mm) were measured for the isolate prior to the series of sequential exposures. ${ }^{*}$ Initial zone of inhibition was not recorded.

The Psendomonas was first evolved to acquire resistance to neomycin. The bacterial strain developed resistance to neomycin at the increments of $5 \mu \mathrm{g} / \mathrm{mL}, 7.5 \mu \mathrm{g} / \mathrm{mL}, 15 \mu \mathrm{g} / \mathrm{mL}, 20 \mu \mathrm{g} / \mathrm{mL}, 25 \mu \mathrm{g} / \mathrm{mL}$, and $30 \mu \mathrm{g} / \mathrm{mL}$ in a sequential order. The optical density measured at $600 \mathrm{~nm}$ reached 1.0 for each increment (Figure 1). As seen in Figure 4a, the zone of inhibition began to disappear as the antibiotic concentration increased. This continued until the Pseudomonas was resistant to neomycin at $30 \mu \mathrm{g} / \mathrm{mL}$ in which there was no longer a zone of inhibition (Figure 4a). This shows that the earlier transfers of the bacterial strain were still 
susceptible to the high dose of neomycin since it only gained resistance at a low, sub-lethal dose. This resulted in a relatively large zone of inhibition (Figure 4a). As this sub-lethal dose that the strain could survive increased, the strain became less susceptible to the antibiotic on disc and, therefore, more resistant, as shown with a smaller inhibition zone (Figure 4a, Table 3). Eventually, this pushed the strain to gain resistance to neomycin at the initially susceptible neomycin concentration yielding bacterial strain 2 .

Next, strain 2 was mutated via culture to gain resistance to ciprofloxacin. Strain 2 developed resistance to ciprofloxacin at increments of $2.5 \mu \mathrm{g} / \mathrm{mL}, 3.5 \mu \mathrm{g} / \mathrm{mL}, 4.5 \mu \mathrm{g} / \mathrm{mL}$, and $5 \mu \mathrm{g} / \mathrm{mL}$. The optical density measured at $600 \mathrm{~nm}$ reached 1.0 for each increment (Figure 2). Figure $4 \mathbf{b}$ depicts this series of sequential exposures and shows that the strain was able to gain resistance due to the lack of a zone of inhibition, so it ultimately evolved into strain 3 in which it was resistant to both neomycin and ciprofloxacin. The measurements of each zone can be found in Table 3.

Lastly, strain 3 underwent a series of sequential exposures until it acquired resistance to imipenem at an initially lethal concentration as well. The bacterial strain developed resistance to imipenem at the increments $1 \mu \mathrm{g} / \mathrm{mL}, 3 \mu \mathrm{g} / \mathrm{mL}, 5 \mu \mathrm{g} / \mathrm{mL}, 7.5$ $\mu \mathrm{g} / \mathrm{mL}$, and $10 \mu \mathrm{g} / \mathrm{mL}$. The optical density measured at $600 \mathrm{~nm}$ reached 1.0 for each increment (Figure 3). As the sub-lethal dose strain 3 was able to survive at was increased, the zone of inhibition induced by the imipenem disc was reduced, suggesting that the strain became less susceptible to the antibiotic disc and therefore more resistant to the drug (Figure 4c, Table 3). Eventually, the strain gained resistance to imipenem at the initially lethal imipenem concentration yielding strain 4 . Evidence of the acquisition of imipenem resistance is shown by the lack of a zone of inhibition after the final transfer (Figure 4c).
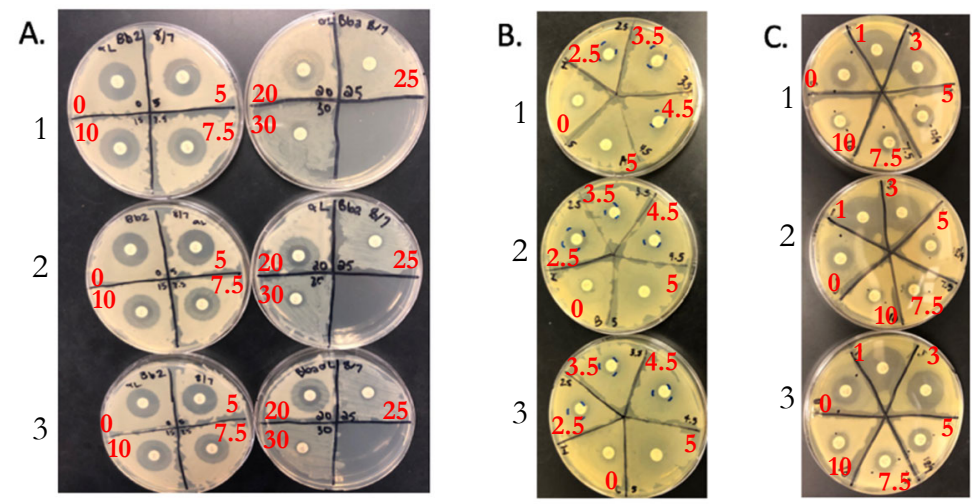

Figure 4. Antibiotic susceptibility disc tests after each series of sequential exposures with each antibiotic. The red number in each pie square is indicative of the antibiotic concentration $(\mu \mathrm{g} / \mathrm{mL})$ in which that strain of bacteria was exposed to and was able to grow. (a) Neomycin; original isolate used for initial inoculation. (b) Ciprofloxacin; neomycin resistant strain $(30 \mu \mathrm{g} / \mathrm{mL})$ used for initial inoculation. The area labeled " $0 \mu \mathrm{g} / \mathrm{mL}$ " indicates that the strain was not exposed to ciprofloxacin, and a neomycin disc was used as a control. (c) Imipenem; neomycin $(30 \mu \mathrm{g} / \mathrm{mL})$ and ciprofloxacin $(5 \mu \mathrm{g} / \mathrm{mL})$ resistant strain used for initial inoculation. The area labeled " $0 \mu \mathrm{g} / \mathrm{mL}$ " indicates that the strain was not exposed to imipenem, and a ciprofloxacin disc was used as a control.

\begin{tabular}{|c|c|c|c|c|c|c|c|c|c|}
\hline \multirow{2}{*}{$\begin{array}{c}\text { [Antibiotic] } \\
\text { Exposure }\end{array}$} & \multicolumn{3}{|c|}{ Neomycin } & \multicolumn{3}{|c|}{ Ciprofloxacin } & \multicolumn{3}{|c|}{ Imipenem } \\
\hline & 1 & 2 & 3 & 1 & 2 & 3 & 1 & 2 & 3 \\
\hline 0 & 20 & 20 & 19 & & & & & & \\
\hline 1 & & & & & & & 26 & 22 & 22 \\
\hline 2.5 & & & & 13 & 14 & 11 & & & \\
\hline 3 & & & & & & & 20 & 20 & 20 \\
\hline 3.5 & & & & 11 & 0 & 11 & & & \\
\hline 4.5 & & & & 8 & 0 & 0 & & & \\
\hline 5 & 20 & 20 & 19 & 0 & 0 & 0 & 15 & 14 & 15 \\
\hline 7.5 & 12 & 14 & 13 & & & & 12 & 12 & 13 \\
\hline 10 & 12 & 13 & 12 & & & & 11 & 9 & 10 \\
\hline 20 & 11 & 12 & 12 & & & & & & \\
\hline 25 & 0 & 0 & 0 & & & & & & \\
\hline 30 & 0 & 0 & 0 & & & & & & \\
\hline
\end{tabular}

Table 3. Zones of inhibition (mm) following antibiotic susceptibility disc tests after each series of sequential exposures with each antibiotic. Boxes shaded grey are not applicable to that antibiotic. Refer to Table 1 to compare to the standards. Bold values indicate resistance gained by the bacterial strain at the initially lethal dose for each drug. 
In order to evaluate whether or not the Pseudomonas was able to maintain resistance to each drug, a multidrug susceptibility disc test was performed. Tryptic soy agar plates with $30 \mu \mathrm{g} / \mathrm{mL}$ neomycin and $10 \mu \mathrm{g} / \mathrm{mL}$ imipenem were made. The bacterial strain at each major point in the study was plated onto these antibiotic TSA plates, and a $5 \mu \mathrm{g} / \mathrm{mL}$ ciprofloxacin disc was used. The results of this disc test are shown in Figure 5. This provides evidence that not only was the Pseudomonas able to gain resistance to each antibiotic, but it was able to maintain this resistance over time. Throughout these series of sequential exposures, there were a total of 15 passages of the bacterial strain to an increased antibiotic concentration. In the neomycin experiment set, there were six passages. In the ciprofloxacin experiment set, there were five passages. Lastly, in the imipenem experiment set, there were four passages.

Taken together, the Pseudomonas isolate was forced to sequentially gain and maintain resistance by shaking with increasing concentrations of each antibiotic (Figure 5d). This acquisition of resistance was not unique to a particular drug class but rather included antibiotics from three different drug classes: a protein synthesis inhibitor (neomycin), a DNA synthesis inhibitor (ciprofloxacin), and a cell wall inhibitor (imipenem).
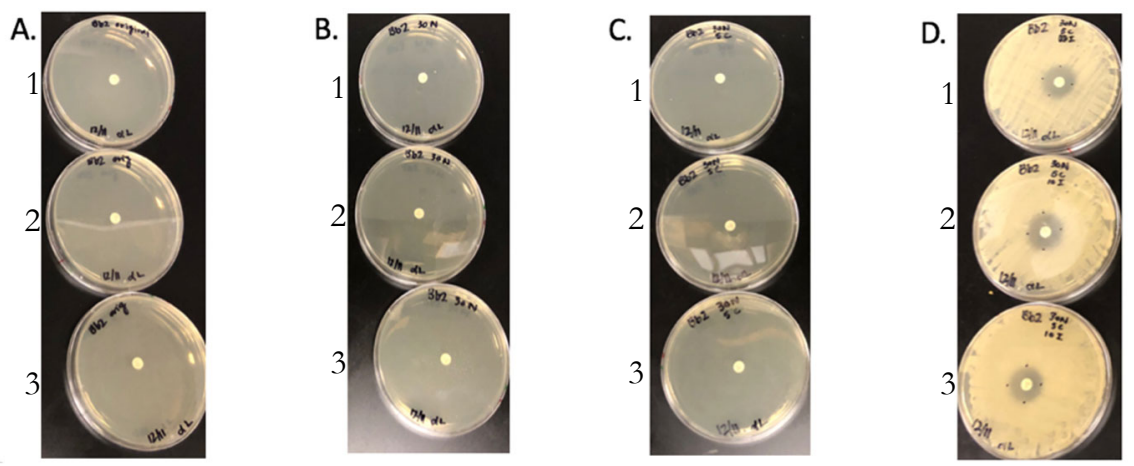

Figure 5. Antibiotic susceptibility disc test $(5 \mu \mathrm{g} / \mathrm{mL}$ ciprofloxacin disc) on antibiotic TSA plate (neomycin $30 \mu \mathrm{g} / \mathrm{mL}$, imipenem $10 \mu \mathrm{g} / \mathrm{mL}$ ) shows that the original isolate successfully acquired MDR to neomycin, imipenem, and ciprofloxacin. (a) Triplicates with strain 1. (b) Triplicates with strain 2. (c) Triplicates with strain 3. (d) Triplicates with strain 4 .

\section{Genome Sequencing}

After the bacterial strain successfully gained resistance to each antibiotic, the genomes of four strains were sequenced and analyzed for SNPs and other genomic changes. MAUVE was used to align genome assemblies for the original and evolved strains in this study. These strains included the original isolate (strain 1), the neomycin resistant strain (strain 2), the neomycin and ciprofloxacin-resistant strain (strain 3), and the neomycin, ciprofloxacin, and imipenem resistant strain (strain 4). While other SNPs were generated from the genome sequencing analysis, Table 4 represents SNPs deemed relevant in the study, including SNPs involving efflux transporters or drug targets of each antibiotic. No large scale insertions or deletions were identified. A total of 1066 mutations between the original isolate and the final MDR strain were found. It is worth noting that the annotation of the gene places an underscore followed by a number when multiple alleles of a gene are identified. This is based on $90 \%$ a.a. similarity, so these are predicted proteins similar to the gene listed.

\begin{tabular}{|c|c|c|c|c|c|c|c|c|}
\hline \multirow[t]{2}{*}{ BP Position } & \multicolumn{4}{|c|}{ Strain } & \multirow[t]{2}{*}{ Mutation } & \multirow[t]{2}{*}{ AA Change } & \multirow[t]{2}{*}{ Gene } & \multirow[t]{2}{*}{ Protein Affected } \\
\hline & 1 & 2 & 3 & 4 & & & & \\
\hline 25477 & & $\mathrm{x}$ & $\mathrm{X}$ & $\mathrm{X}$ & G to $T$ & Leu 40 to Ile & \multirow[t]{2}{*}{ emrB } & \multirow{2}{*}{$\begin{array}{c}\text { EmrAB-TolC multidrug efflux transport system - } \\
\text { membrane subunit }\end{array}$} \\
\hline 25506 & & $\mathrm{X}$ & $\mathrm{X}$ & $\mathrm{X}$ & $\mathrm{C}$ to $\mathrm{G}$ & Ser 30 to Thr & & \\
\hline 10233 & & $\mathrm{X}$ & $\mathrm{X}$ & $\mathrm{X}$ & C to $\mathrm{G}$ & Ala 27 to Pro & \multirow[t]{4}{*}{$m d t C$} & \multirow{4}{*}{$\begin{array}{c}\text { MdtABC-TolC multidrug efflux transport system - } \\
\text { membrane subunit }\end{array}$} \\
\hline 10238 & & $\mathrm{X}$ & $\mathrm{X}$ & $\mathrm{X}$ & $\mathrm{C}$ to $\mathrm{A}$ & $\operatorname{Arg} 25$ to Leu & & \\
\hline 10279 & & $\mathrm{X}$ & $\mathrm{X}$ & $\mathrm{X}$ & T to $G$ & Lys 11 to Asn & & \\
\hline 10289 & & $\mathrm{X}$ & $\mathrm{X}$ & $\mathrm{X}$ & $\mathrm{C}$ to $\mathrm{A}$ & Gly 8 to $\mathrm{Val}$ & & \\
\hline 39721 & & & $\mathrm{X}$ & $\mathrm{X}$ & A to $\mathrm{T}$ & Leu 55 to $\mathrm{Gln}$ & gyrA & DNA gyrase, subunit A \\
\hline 99267 & & & $\mathrm{X}$ & $\mathrm{X}$ & A to $\mathrm{C}$ & Glu 469 to Asp & $g y r B$ & DNA gyrase, subunit B \\
\hline 36359 & & & $\mathrm{X}$ & $\mathrm{X}$ & A to $\mathrm{T}$ & Ter 562 to $\mathrm{Tyr}$ & parC & Topoisomerase IV subunit A \\
\hline 42508 & & & & $\mathrm{X}$ & C to $G$ & Ala 546 to Pro & $m d t C$ & $\begin{array}{c}\text { MdtABC-TolC multidrug efflux transport system - } \\
\text { membrane subunit }\end{array}$ \\
\hline 78781 & & & & $\mathrm{X}$ & $\mathrm{G}$ to $\mathrm{T}$ & Asp 617 to Glu & $\operatorname{mrd} A$ & $\begin{array}{c}\text { Cell shape; peptidoglycan synthetase; penicillin-binding } \\
\text { protein } 2\end{array}$ \\
\hline
\end{tabular}

Table 4. Genome mapping reveals SNPs acquired and maintained in response to each antibiotic stressor. Strain 1 refers to the original isolate. Strain 2 refers to the neomycin resistant strain. Strain 3 refers to the neomycin and ciprofloxacin-resistant strain. Strain 4 refers to the neomycin, ciprofloxacin, and imipenem resistant strain. An "X" indicates that the strain has the corresponding mutation. Under the amino acid change column, "ter" refers to termination. 
While neomycin binds to the 30s subunit of the bacterial ribosome, thereby inhibiting protein synthesis, there were no mutations in the neomycin resistant strain that pertained to the 30s ribosomal subunit (Table 4). However, mutations were seen in the DNA coding for both the EmrAB-TolC multidrug efflux transport system and the MdtABC-TolC multidrug efflux transport systemboth acquired mutations in the membrane subunit of these efflux transporters (Table 4). These transport systems are multiprotein complexes that involve both the inner and outer membranes of gram-negative bacteria. ${ }^{14}$ The TolC portion of the system is embedded in the outer membrane and works in synergy with a variety of active transporters, including the EmrAB or MdtABC transmembrane proteins that lie in the inner membrane. ${ }^{14}$ TolC acts as a channel that transports molecules out of the periplasm that is pumped in by the active transporters EmrAB or MdtABC. ${ }^{14}$ TolC is capable of transporting a wide variety of molecules, including antibiotics, antibacterial peptides, bile salts, and toxins. ${ }^{14}$ Since TolC is already versatile; it is more likely that the selective pressure of neomycin resulted in mutations in the EmrAB and MdtABC domains of the efflux transporters. The acquisition of mutations in these genes suggests that the original isolate improved its ability to actively pump antibiotics out of its cell through mutations to these transporters. Active efflux is one of the most common mechanisms of antibiotic resistance, so this mechanism likely played a role in the acquisition of resistance to neomycin while undergoing neomycin as a selective pressure.

The two missense mutations in the EmrAB-TolC transporter resulted in amino acid changes, but the mutations yielded structurally and chemically similar amino acids to the original protein. At the base pair position of 25477 , leucine 40 was exchanged for isoleucine (Table 4). Both of these amino acids are hydrophobic but have subtle structural changes. Similarly, the other missense mutation in this protein complex was serine 30 to threonine (Table 4). Both of these amino acids are polar and slightly different in structure.

Additionally, there were four missense mutations in the MdtABC-TolC multidrug efflux transport system. At the base pair position 10233, alanine 27 was changed to proline. At the base pair position 10238, arginine 25 was changed to leucine. At the base pair position 10279, lysine 11 was changed to asparagine. Lastly, at the base pair position 10289, glycine 8 was changed to valine. (Table 4) The effect of these mutations on protein structure is unclear, but due to the efflux transport system's function, they may have improved the abilities of the efflux transport system to pump neomycin out of the cell.

After gaining resistance to neomycin through point mutations, the strain further mutated under the selective pressure of ciprofloxacin until it gained ciprofloxacin resistance. Strain 3 maintained the SNPs in the EmrAB-TolC multidrug efflux transport system and the MdtABC-TolC multidrug efflux transport system acquired by strain 2 under the neomycin selective pressure (Table 4). In addition to maintaining these mutations, the strain acquired SNPs in the genes encoding for DNA gyrase and topoisomerase IV. These are likely significant mutations since ciprofloxacin directly targets both of these enzymes in order to kill the bacterial cell. Ciprofloxacin is able to inhibit DNA replication through its inhibition of these proteins.

Ciprofloxacin resistance in bacteria is attributed to an increase in efflux transporter expression and mutations of the enzymes DNA gyrase and topoisomerase IV that lower the drug's binding affinity to these enzymes. ${ }^{12}$ SNPs in both DNA gyrase and topoisomerase IV were found (Table 4). There was a mutation to the DNA gyrase subunit B in which glutamic acid 469 was changed to aspartic acid. This mutation has been found to be a key mutation in the lowering of the binding affinity of ciprofloxacin to DNA gyrase in other studies that have evaluated the acquisition of ciprofloxacin resistance in Pseudomonas aeruginosa. ${ }^{15}$ Additionally, leucine 55 was found to be mutated to glutamine in DNA gyrase subunit A (Table 4). The leucine 55 mutation has not been identified as a relevant mutation in other resistant Pseudomonas species, so it may be a novel mutation that aids in lowering ciprofloxacin's binding affinity therefore contributing to the acquisition of ciprofloxacin resistance. There was also a mutation in the topoisomerase IV gene in which the termination sequence was mutated to tyrosine (Table 4). The topoisomerase IV mutation function is unknown but may affect the structure of the enzyme leading to lower ciprofloxacin binding affinity.

Lastly, strain 3 gained imipenem resistance through the imipenem stressor. Upon acquisition of imipenem resistance by strain 3 , strain 4 was developed. The previously identified mutations that were acquired under both neomycin and ciprofloxacin pressures were conserved in strain 4, so the bacteria were able to maintain these genetic changes (Table 4). The MdtABC-TolC multidrug efflux transport system was originally mutated in the $m d t C_{-} 4$ gene when the strain was exposed to neomycin (Table 4). After imipenem exposure, the strain retained these original mutations to the complex and gained another mutation to this complex, but in a different gene. A SNP was found in which alanine 546 was changed to proline in the $m d t C \_1$ gene (Table 4). It is unknown what this exact mutation did to the protein and the transport system as a whole. However, an additional mutation to the MdtABC-TolC multidrug efflux transport system complex suggests that this efflux transporter plays a role in antibiotic resistance since the strain continued to mutate and maintain previous mutations to this efflux transporter as it adapted in response to the different antibiotic stressors. 
The MDR strain also acquired a mutation at the 78781 base pair position in which aspartic acid 617 was changed to glutamic acid, which lies within the $m r d A \_1$ gene (Table 4). This gene codes for penicillin-binding protein two, which plays a role in peptidoglycan biosynthesis. Imipenem's mechanism of action is to weaken the bacterial cell wall by binding penicillin-binding proteins to prevent peptidoglycan synthesis and weaken the cell wall. ${ }^{8}$ The mutation in the $m r d A \_1$ gene has not been an identified mutation in other imipenem resistant Pseudomonas species. However, mutations in this gene have been found in other bacterial strains that are resistant to the cell wall inhibitor class of drugs. ${ }^{16}$ Due to the nature of the mrdA_1 gene mutation in that both amino acids have similar structures and chemical properties, it cannot be concluded that this mutation was a key change that resulted in the gaining of imipenem resistance. It is plausible that the mutation may affect imipenem's ability to bind penicillinbinding protein two, leading to the resistance seen in strain 4. It is also possible that the combination of many mutations rather than the sole mrdA_1 gene mutation led to the resistance observed.

Many previous studies have addressed and identified key mutations in the acquisition of MDR in Psendomonas species. In comparison to previous studies, many of the identified mutations appear to be novel in this study. Previous studies have found the MexXY multidrug efflux pump system to be a commonly expressed efflux pump in Psendomonas species as well as a commonly mutated efflux pump in other neomycin resistant Pseudomonas species. ${ }^{17}$ While no mutations to the MexXY multidrug efflux pump were found in this study, mutations to other efflux pumps were identified. In response to ciprofloxacin, previous studies have found a variety of mutations that contribute to the acquisition of ciprofloxacin resistance in other Psendomonas species. The common mutations identified were mutations to the genes encoding the enzymes gyrase and topoisomerase IV. The mutation in the gyrB gene found in this study has been identified as a key mutation in the acquisition of resistance to ciprofloxacin in other Pseudomonas species by other studies. ${ }^{15}$ This result suggests the importance of the gene $g r y B$ in ciprofloxacin resistance. The other mutations to gyrase and topoisomerase IV enzymes that were identified by this study have not been previously identified, suggesting that the mutations may be novel. Lastly, in response to imipenem, previous studies have found mutations in the mrdA_1 gene. While there was a mutation identified in the $m r d A \_1$ gene by this study, the mutation found was not a previously identified mutation suggesting another potential novel mutation.

Antibiotics are widely used in both medical and agricultural industries. The overuse and abuse of these drugs have been linked to the emergence and persistence of antibiotic-resistant bacteria and the genes that facilitate such resistance. In this study, we evaluated an environmental microbe's ability to acquire resistance to multiple antibiotic drugs. Our data suggest that this environmental microbe could become resistant to not only one but four different types of antibiotic drugs under selective pressure. This phenomenon of acquiring resistance is not novel. However, this study highlights the importance of researching how resistance can be acquired in environmental rather than clinical microbes. As such microbes thrive in the environment, they also have the ability to pass their antibiotic-resistant genes to neighboring bacterial species through horizontal gene transfer. It is plausible to suggest that some of these environmental microbes are potential human pathogens and therefore, will be very difficult infections to treat with common antibiotic drugs. Antibiotic resistance is a worldwide public health crisis. Further studies must be conducted to determine the magnitude of selective pressure required for both clinical and environmental bacteria to acquire resistance and how pollution of antibiotic drugs in the environment can be mitigated.

\section{CONCLUSIONS}

The Pseudomonas isolate successfully gained and maintained resistance to neomycin, ciprofloxacin, and imipenem at initially lethal doses to the organism through a series of sequential exposures. These data provide evidence that the use of multiple antibiotics in local populations may contribute to MDR development. Through genome alignment and analysis, a variety of SNPs were identified between the original isolate and the MDR isolate. These SNPs suggest that efflux transporters and mutations in drug target proteins play vital roles in developing drug resistance.

\section{ACKNOWLEDGEMENTS}

Thank you to the College of St. Benedict St. John's University's for the support on my research endeavors. Additionally, the authors acknowledge the CSBSJU FDRC for funds supplied to D.M. A thanks to Sarah Rhodes for her contributions to the project. Additionally, thank you to Dr. Elyse Krautkramer for guidance and contributions to the project. Finally, we thank the technical work of Cristian Flores-Figueroa and Jeannette Munoz-Aguayo at the Mid-Central Research and Outreach Center. Bioinformatics were supported using tools available from the Minnesota Supercomputing Institute. Genome sequences are available from the authors (D.M.) upon request.

\section{REFERENCES}

1. Martinez, J. (2008) Antibiotics and Antibiotic Resistance Genes in Natural Environments, Science 18, 365-367. doi:10.1126/ science. 1159483

2. Davies, J., and Davies, D. (2010) Origins and Evolution of Antibiotic Resistance, Mol Biol Rev 74, 417-433. doi:10.1128/MMBR.00016-10 
3. United States Department of Labor, Healthcare Wide Hazards: Multi-drug Resistant Organisms, https:// www.osha.gov/SLTC/etools/hospital/ hazards/mro/mro.html (accessed May 2020)

4. Bonomo, R. (2006) Mechanisms of Multidrug Resistance in Acinetobacter Species and Pseudomonas aeruginosa, Clin Infect Dis 43, 49-56. doi:10.1086/504477

5. Munita, J., and Arias, C. (2016) Mechanisms of Antibiotic Resistance, Microbiol Spectr 4, 1-37. doi:10.1128/microbiolspec.VMBF0016-2015

6. CDC. (2019). Antibiotic Resistance Threats in the United States. Atlanta, GA: U.S. Department of Health and Human Services, CDC.

7. Hager, S., Jensen, E., Johnson, T., and Mitchell, D. (2017) Adaption of Escherichia coli to Antibiotic Cycling via Single Nucleotide Polymorphisms, Am J Undergrad Res 14, 17-34. doi:10.33697/ajur.2017.001

8. Van Hoek, A., Mevius, D., Guerra, B., Mullany, P., Roberts, A., and Aarts, H. (2011) Acquired antibiotic resistance genes: an overview, Front Microbiol 2, 1-27. doi:10.3389/fmicb.2011.00203

9. Du, D., Wang-Kan, X., Neuberger, A., Veen, H., Pos, K., Piddock, L., and Luisi, B. (2018) Multidrug efflux pumps: structure, function, and regulation, Nat Rev Microbiol 16, 523-539. doi:10.1038/s41579-018-0048-6

10. Mehta, R., Champney, S. (2003) Neomycin and Paromomycin Inhibit 30S Ribosomal Subunit Assembly in Staphylococcus aureus, Curr Microbiol 47, 237-243. doi:10.1007/s00284-002-3945-9

11. Bankevich, A., Nurk, S., Antipov, D., Gurevich, A., Dvorkin, M., Kulikov, A., Lesin, V., Nikolenko, S., Pham, S., Prjibelski, A., Pyshkin, A., Sirotkin, A., Vyahhi, N., Tesler, G., Alekseyev, M., Pevzner, P. (2012) SPAdes: a new genome assembly algorithm and its applications to single-cell sequencing, J Comput Biol 19, 455-77. doi:10.1089/cmb.2012.0021

12. Seemann, T. (2014) Prokka: rapid prokaryotic genome annotation, Bioinformatics 30, 2068-9. doi:10.1093/bioinformatics/btu153

13. Darling, A., Mau, B., Blattner, F., Perna, N. (2004) Mauve: multiple alignment of conserved genomic sequence with rearrangements, Genome Res 14, 1394-1403. doi:10.1101/gr.2289704

14. Tikhonova, E., Dastidar, V., Rybenkov, V., and Zgurskaya, H. (2009) Kinetic control of TolC recruitment by multidrug efflux complexes, PNAS 106, 16416-16421. doi:10.1073/pnas.0906601106

15. Rehman, A., Patrick, W., and Lamont, I. (2018) Mechanisms of ciprofloxacin resistance in Pseudomonas aeruginosa: new approaches to an old problem, J Med Microbiol 68, 1-10. doi:10.1099/jmm.0.000873

16. Ranjitkar, S., Reck, F., Ke, X., Zhu, Q., McEnroe, G., Lopez, S., Dean, C. (2019) Identification of Mutations in the mrdA Gene Encoding PBP2 That Reduce Carbapenem and Diazabicyclooctane Susceptibility of Escherichia coli Clinical Isolates with Mutations in ftsI (PBP3) and Which Carry blaNDM-1, mSphere 4, 1-6. doi:10.1128/mSphere.00074-19

17. Chuanchuen, R., Wannaprasat, W., Ajarivakhajorn, K., Schweizer, H. (2008) Role of the MexXY multidrug efflux pump in moderate aminoglycoside resistance in Pseudomonas aeruginosa isolates from Pseudomonas mastitis, Microbiol Immunol 52, $392-398$. doi:10.1111/j.1348.0421.2008.00051.x

\section{ABOUT STUDENT AUTHOR}

Allison Grodnick graduated from the College of St. Benedict in St. Joseph, MN in May 2020. She earned a Bachelor's of Art with a major in Biochemistry. In the fall of 2021, she will begin graduate school at the University of Minnesota through their Biochemistry, Molecular Biology, and Biophysics Ph.D. program.

\section{PRESS SUMMARY}

As an unintentional result of the extensive use of antibiotics in healthcare and agriculture, antibiotics have become a prevalent selective pressure on bacteria. This pressure forces bacteria to evolve drug resistance through mutations in order to survive. This study aims to evaluate the acquisition of resistance to multiple antibiotics by an initially susceptible isolated bacterium from a Minnesota forest environment. Neomycin, ciprofloxacin, and imipenem were the selected antibiotics used to determine if this isolate could become resistant over time and exposure. The initial isolate acquired resistance to all three selected antibiotics. Single nucleotide polymorphisms between the original isolate and the final resistant strain were identified and are suspected to play a role in the isolate's gain of antibiotic resistance. Insight into how bacteria gain antibiotic resistance is essential in addressing this rising health issue. 


\title{
Stripping Material from a Supported Lipid Bilayer with High Speed Buffer Flow
}

\author{
Michael J. Ornstead, Ruth Hunter, Mason L. Valentine, Cameron Cooper, Stephen K. Smith, \& Christopher F. Monson* \\ Department of Physical Science, Southern Utab University, Cedar City, UT \\ bttps:// doi.org/10.33697/ajur.2020.027 \\ Students:mikeornstead@yahoo.com,kruth.bunter@gmail.com,masonlvalentine@utexas.edu,cam_cooper90@hotmail.com, \\ stephenksmith263@gmail.com \\ Mentor:christophermonson1@suu.edu
}

\begin{abstract}
A microfluidic device was created and used to demonstrate that supported lipid bilayers can be deposited on clean glass slides and removed using high velocity buffer flow (1-4 $\mathrm{m} / \mathrm{s}$ linear velocity). This was accomplished by forcing the flow through a microfluidic channel covering an annealed glass coverslip bearing a supported lipid bilayer (SLB). The removal of bilayer material was monitored via fluorescence microscopy, and two basic regimes were observed: at 1-2 m/s smaller areas were stripped, while at $3-4 \mathrm{~m} / \mathrm{s}$ larger areas were stripped. SLB removal was verified by two means. First, lipid vesicles labeled with a different fluorescent dye were added to the device and filled in holes left by the removal of the original SLB, allowing stripping to be verified visually. Second, the solutions obtained from stripping were concentrated and the fluorescence in the concentrates was measured. The ability to strip SLB from glass provides a relatively gentle method of creating spatially inhomogeneous SLB, which could be a useful tool in the continued investigation of membrane properties and components.
\end{abstract}

\section{KEYWORDS}

Supported Lipid Bilayer; Membrane Vesicle; Microfluidic Device

\section{INTRODUCTION}

A supported lipid bilayer (SLB) consists of two leaflets of amphiphilic molecules, typically phospholipids, supported by a clean, flat substrate, often glass. ${ }^{1,2}$ SLBs retain many of the properties of biological lipid membranes such as those found in cells. ${ }^{3}$ Molecules in a SLB retain their lateral fluidity, and the amphiphilic nature of SLBs causes molecules to assume configurations similar to those assumed in native cell membranes, thus they are of interest as native membrane mimics. ${ }^{4-7}$ Most SLBs are composed primarily of phospholipids, which have a hydrophilic head and a hydrophobic tail, but other, similarly structured lipids can also be used, and molecules like cholesterol can be added to modify the properties of the bilayer. ${ }^{8-11}$

SLBs can be patterned on their substrates using a variety of methods. For example, barriers can be placed photolithographically or by other means to control SLB formation. ${ }^{12-16}$ Alternatively, patterns can be created after a bilayer is formed, for example by scratching to create a barrier, applying an electric field to induce movement of charged lipids or proteins, using DNA or microbeads. ${ }^{17-23}$ Patterns in SLBs can also be created using polymer stencils, applied either before a bilayer is deposited or used after the bilayer has been deposited to lift off a section of bilayer. ${ }^{24,25}$ Such patterns can be used to facilitate the purification of membrane components.

One of the principal uses for SLBs is as model cell membranes, particularly in the study of membrane proteins. Membrane proteins are integral to many cell functions including signaling, metabolism, and cell structure. ${ }^{26}$ Despite their importance, membrane proteins are challenging to work with due to their amphiphilic nature - most membrane proteins are only stable and active in a lipid bilayer ${ }^{27-29}$ Despite these challenges, there are numerous examples of work that has been done with membrane proteins and peptides, including spectroscopy, ${ }^{30}$ crystal structure, ${ }^{31}$ and membrane organization. ${ }^{32}$ Several membrane protein methods have been developed that use supported lipid bilayers. ${ }^{32-36}$

Among SLB techniques that show great promise with membrane proteins are those that involve separations. Several separation methods have been developed that rely on lipid and protein motion in electric fields, including line electrophoresis and electrophoretic-electroosmotic flow. ${ }^{37-40}$ In other separation methods bulk flow, ${ }^{41-42}$ lipid curvature, ${ }^{43}$ Brownian motion, ${ }^{44}$ or surface acoustic waves ${ }^{45}$ are used to sort membrane components. To allow work with membrane proteins, these techniques can be combined with molecular cushions, which minimize protein denaturation on the support surface. ${ }^{33,34,46-52}$ 
While these techniques show great promise, particularly in combination, they have limitations with certain proteins, particularly proteins that transport large molecules, as the space on the supported side of the bilayer is insufficient to allow for the transport of a large molecule. We introduce a method of removing SLB components in an effort to create vesicles of materials after, for example, they have been purified by SLB-based techniques. Other methods have been developed to remove SLB components, but they typically use detergents and thus would remove any proteins from the membrane environment. ${ }^{53}$ Another method growing in popularity uses a mixture of styrene and maleic acid, which is less denaturing for proteins. ${ }^{54,55}$ Our method involves high-speed buffer flow, as will be discussed below.

\section{MATERIALS AND PROCEDURES}

\section{Lipid vesicle preparation}

Lipid vesicles were prepared using a modified version of the freeze-thaw extrusion method. ${ }^{66-57}$ Dried lipids were dissolved in chloroform and mixed in appropriate volumes to produce the desired lipid compositions (1\% fluorophore labeled lipid in 1palmitoyl-2-oleoyl-glycero-3-phosphocholine (POPC), Avanti Polar Lipids, Alabaster, Alabama). The fluorophores used were fluorescein-labeled 1,2-dioleoyl-sn-glycero-3-phosphoethanolamine (Fl-DOPE, Avanti Polar Lipids, Alabaster, Alabama) and Texas Red-labeled 1,2-dihexadecanoyl-sn-glycero-3-phosphoethanolamine (TR-DHPE, Biotium, Inc., Fremont, California). The chloroform was evaporated in a stream of air and then in a vacuum at 3 torr for at least 4 hours. The dried lipids were hydrated with a tris(hydroxymethyl)aminomethane (Tris, Fisher Scientific) buffer solution ( $0.1 \mathrm{M} \mathrm{NaCl}, 0.02 \mathrm{M}$ Tris, $\mathrm{pH}$ 7.4) while being continuously but gently swirled. The vesicles were then extruded using a mini-extruder (Avanti Polar Lipids, Alabaster, Alabama) with a $100 \mathrm{~nm}$ pore track-etched membrane (Whatman, Maidstone, United Kingdom). The solution was forced through the filter ten times in each direction, for twenty total passes, and then stored at $4{ }^{\circ} \mathrm{C}$ until use.

\section{Glass Cleaning}

Glass coverslips (24 x $50 \mathrm{~mm}$, Fischer) were cleaned by boiling in 7x detergent solution (MP Biomedical) at around 1:10 dilution with water for an hour, followed by rinsing with deionized water $(18 \mathrm{M} \Omega)$ for several minutes. The slides were annealed at $530^{\circ} \mathrm{C}$ for several hours and then stored in a clean slide box until use..$^{58-59}$

\section{Device Fabrication}

Microfluidic devices were fabricated individually in a multi-step process. A box was first constructed out of glass using superglue to bond pieces together. The bottom glass piece had two $8 \times 8 \mathrm{~mm} 150 \mu \mathrm{m}$ thick glass pieces glued near its center, about $10 \mathrm{~mm}$ apart. These were the "landing pads." A second piece of glass, about $4 \mathrm{~mm}$ wide and $12 \mathrm{~mm}$ long, was placed between these two glass pieces (but not glued to them), held off the base by the landing pads. Enough polydimethylsiloxane (PDMS, Silgard 184, Dow Corning) to just cover the landing pads was then poured into the box, and the device was baked at $110{ }^{\circ} \mathrm{C}$ for 10 min. Metal wire was used to create a junction between the micro-scale portion of the device and the supply tubes - the wire was carefully placed such that it ended over the landing pads and extended to the outer edge of the box. Another $\sim 2 \mathrm{~mm}$ layer of PDMS was then added to the device and hardened at $110^{\circ} \mathrm{C}$ for $10 \mathrm{~min}$. Pieces of $1 \mathrm{~mm}$ thick glass slides were then added to the device as internal stiffeners, and an additional $\sim 3 \mathrm{~mm}$ layer of PDMS was added. Another two layers of stiffening glass in $\sim 2 \mathrm{~mm}$ each of PDMS (four $\mathrm{mm}$ total) were baked in the device, and a final layer of PDMS was poured over the device until the device height was around $1.5 \mathrm{~cm}$, at which point the device was baked overnight. The glass box was then removed, which also removed the landing pads. Two exacto knife blades were taped together and used to cut a straight channel connecting the two landing pads, as shown in Figure 1.

\section{Stripping Procedure}

A clean glass coverslip was clamped to the device with a $1 \mathrm{~mm}$ thick glass slide behind it for additional strength, and the device was soaked in DI water in a vacuum jar at 10 torr for at least 30 minutes to remove air from the device. The device was then loaded with $5 \mathrm{mM}$ Tris (no NaCl, $\mathrm{pH}$ 7.5) buffer solution, following which it was loaded with 60 microliters of 1\% TR-DHPE or $1 \%$ Fl-DOPE lipid vesicles (in POPC and Tris). Following loading, the device was allowed to sit for at least 30 minutes to allow for the formation of a supported lipid bilayer (SLB). The device was then rinsed with $5 \mathrm{mM}$ Tris buffer solution at a rate of 0.2 $\mathrm{m} / \mathrm{s}$, for at least 10 minutes, in order to remove excess vesicles from the device. The flow rate was then increased to between 1.1 and $4.2 \mathrm{~m} / \mathrm{s}$ to strip material from the SLB. Stripping in the channel was monitored via fluorescence microscopy.

\section{Backefilling Procedure}

Following a successful stripping attempt, the device was loaded with 60 microliters of the fluorescent lipid not used initially (TRDHPE or Fl-DOPE) in POPC and allowed to sit for at least five minutes for backfilling to occur. The device was then rinsed with $5 \mathrm{mM}$ Tris buffer at $0.5 \mathrm{~mL} / \mathrm{min}$ to remove excess vesicles from the device, and backfilling was evaluated using fluorescence microscopy. 

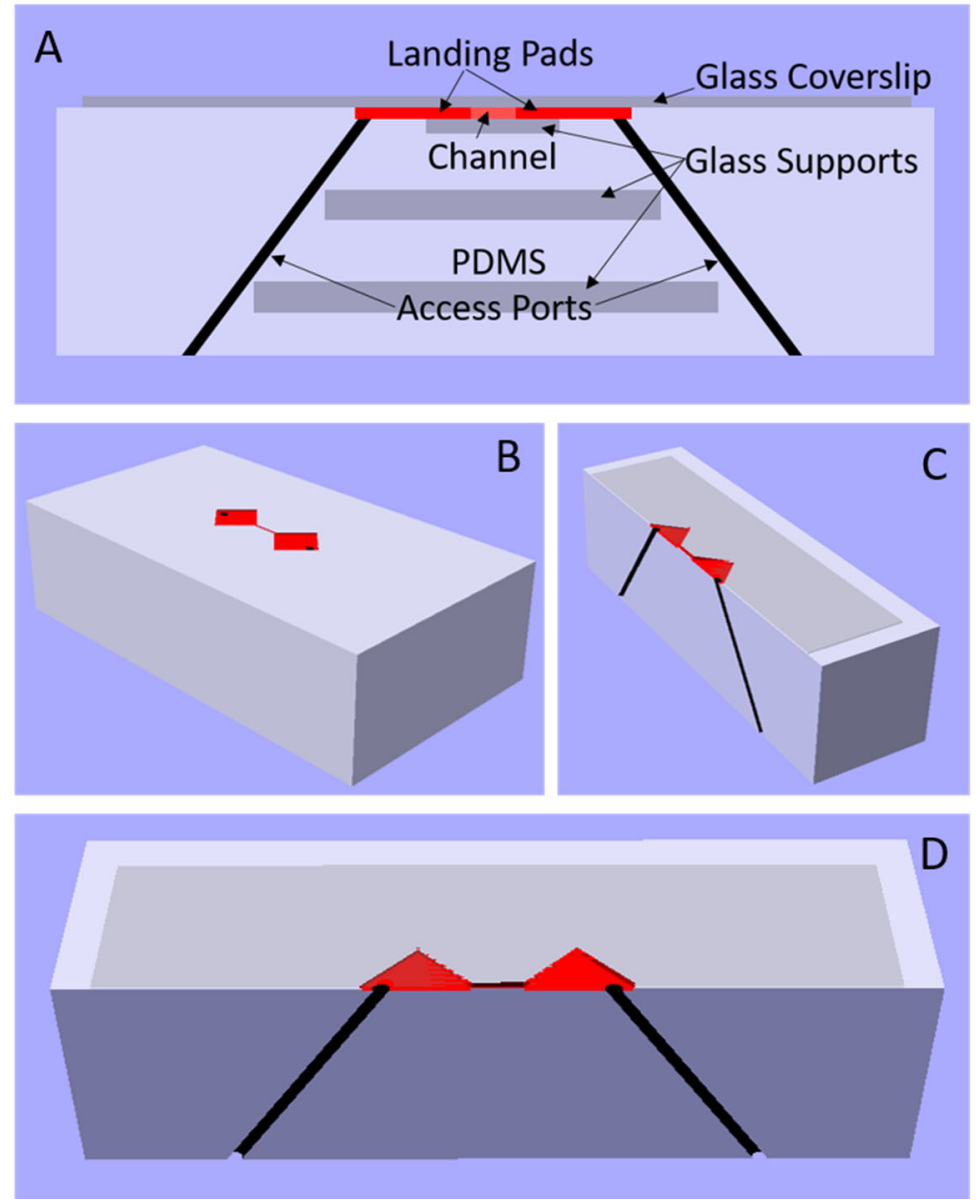

Figure 1. Device illustration. Panel A shows a labeled cutaway side view of the device. The bilayer is localized to the landing pads and the channel (attached to the removable glass coverslip). The access ports carry solution to and from the bilayer. There is a piece of glass coverslip at the bottom of the channel, and four $1 \mathrm{~mm}$ thick pieces of glass slides inside the device (only two are shown, the other two are between the two shown and are on either side of the access ports). B shows the entire device without a coverslip, and $\mathrm{C}$ and $\mathrm{D}$ show cutaway perspective views of the device with a coverslip.

\section{RESULTS}

The SLBs initially appeared smooth after rinsing as expected (Figure 2, 0 min). In Figure 2, the bright area is the fluorescently labeled lipid bilayer (labeled with 1\% TR-DHPE in POPC). The areas that are initially dark are those areas of glass bonded to the PDMS device and that thus bear no lipids. After solution was flowed over the bilayer at a high speed, dark defects started to occur, often in a linear pattern (Figure 2, $5 \mathrm{~min}$ ). These defects would commonly grow with time. To verify that the defects were not due to an elastic lateral compression of the lipids, the defects were observed both while buffer was flowing and then with no flow, and no change was observed. The defects (darker areas appearing in the light areas) are at least in part due to lipids being
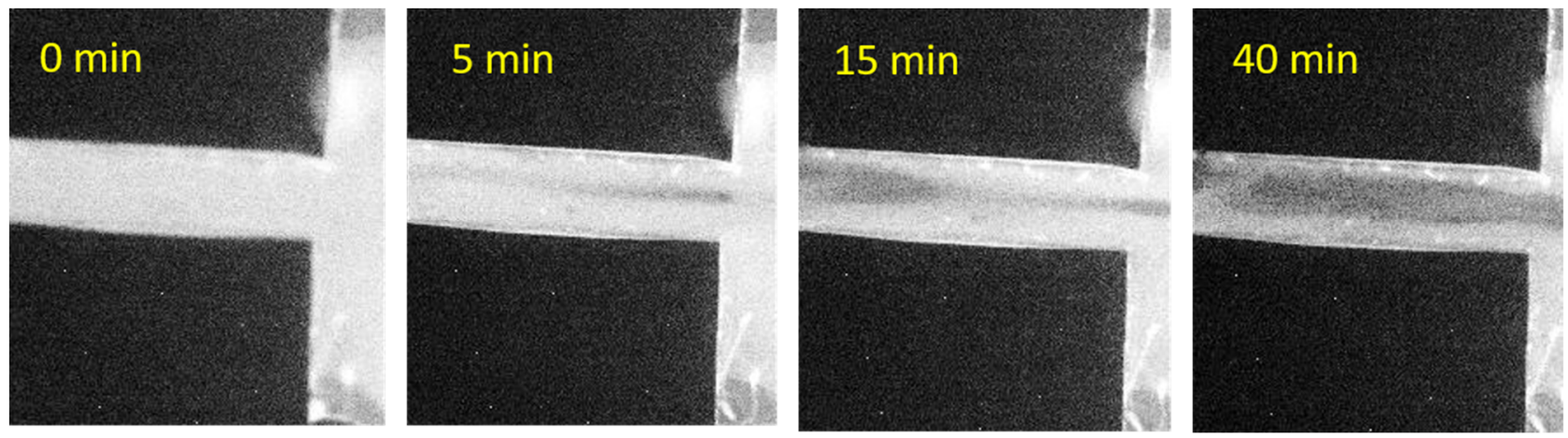

Figure 2. Stripping a SLB from a glass surface as seen by fluorescence microscopy. As can be seen, an initially smooth bilayer is removed from much of the central channel area over the course of 40 minutes at a flow rate of around $1.1 \mathrm{~m} / \mathrm{s}$. 
stripped from the solid support and returned to the aqueous phase (referred to hereafter as eluent, in analogy to chromatography), presumably as micelles or vesicles.

As the stripped area was observed to increase with time and the rate of stripping was observed to depend on the flow rate, the efficiency of various flow rates could be compared, as shown in Figure 3. As might be expected, while SLB stripping occurred at both slower and faster flow rates, the efficiency of the stripping differed. At low flow rates $(1-2 \mathrm{~m} / \mathrm{s}$ linear flow rate, or 3-4 $\mathrm{mL} / \mathrm{min}$ volumetric flow rate), the stripping happened more slowly (taking on average 40 minutes to strip the maximum area that could be stripped - additional time did not result in further bilayer stripping) but the efficiency in terms of volume of eluent used and thus bilayer material concentration in the eluent was maximized. On the other hand, at high flow rates $(\sim 4 \mathrm{~m} / \mathrm{s}$ linear flow rate or $10 \mathrm{~mL} / \mathrm{min}$ volumetric flow rate) the stripping was faster, although it used somewhat more eluent. Most of the initial runs were performed at low flow rates, while later runs were typically at higher flow rates. It should also be noted that higher flow rates subjected the device to significant stress and that device failure was more common at higher flow rates. We also tested solutions of different ionic strengths: from $1 \mathrm{M} \mathrm{NaCl} / 0.01 \mathrm{M}$ Tris to deionized water, and did not observe any appreciable effects on stripping time, area, or efficiency.
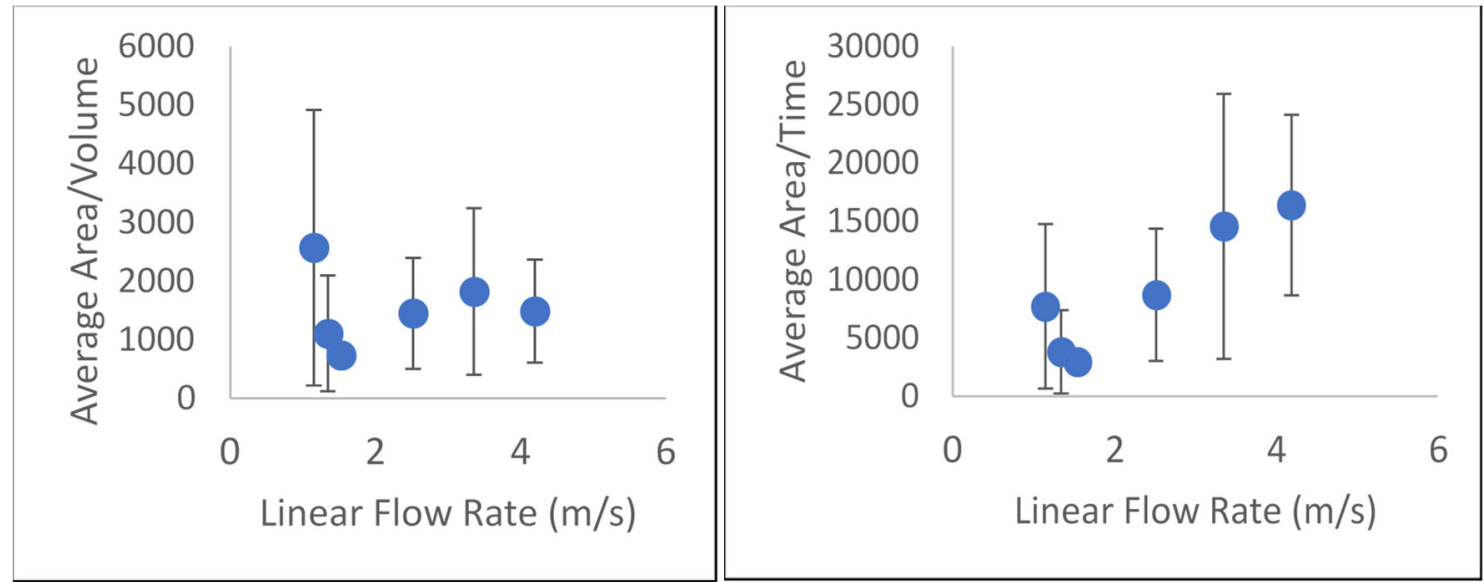

Figure 3. Stripping efficiency as a function of flow rate. Stripping efficiency is illustrated as the average area stripped per volume of solution (in $\mu \mathrm{m}^{2} / \mathrm{mL}$ ) and average area stripped per time (in $\mu \mathrm{m}^{2} / \mathrm{min}$ ), both as a function of linear flow rate of buffer over the SLB (in $\mathrm{m} / \mathrm{s}$ ). As can be seen in the graphs, the stripping is slightly more efficient per volume (resulting in a higher concentration of stripped lipids) at lower flow rates but more time efficient at higher flow rates. Error bars are standard deviations.

To verify that the observed darkening was indeed lipid loss to the eluent as opposed to lipid migration or another analogous process, several experiments were performed. First, additional lipids labeled with a different fluorophore were added after a stripping run was performed. If the lipids initially on the glass had been completely removed by the solution flow, the bare glass surface should induce bilayer reformation with the new vesicles. Thus stripping runs were performed on a 1\% Fl-DOPE in POPC SLB and then 1\% TR-DHPE in POPC vesicles were added to the stripped bilayer. This is illustrated in Figure 4. The bilayer initially is an even green (due to the Fl-DOPE). After the stripping, there are dark regions in the bilayer where the initial bilayer has been stripped away (Figure 4 B). After TR-DHPE labeled lipids are added, many of these dark areas are observed to fluoresce red, demonstrating that the TR-DHPE labeled lipids have formed a bilayer on the glass.

This method of removing and backfilling lipids may be useful for more than just demonstrating that stripping has occurred. The commonly employed methods for patterning bilayers when two patches of contiguous bilayer are required to be different is either to put one bilayer down, scratch to remove part of the bilayer, and then backfill the scratched region with additional lipids ${ }^{37}$ or to use polymer blockers to prevent a region from being filled with lipids and then remove the blockers and fill the space with a second type of lipid. ${ }^{60}$ Using high speed buffer flow to remove SLB should be more gentle than scratching the surface ${ }^{37}$ and not potentially leave a residue like polymer blockers. ${ }^{60}$ Thus, it could be a more effective way to create an SLB with two compositionally distinct regions. We attempted to determine whether this would be the case by looking at fluorophore-labeled lipid diffusion from one region of the bilayer to the other, however our results were inconclusive - it appeared that some regions demonstrated diffusion, but not all. 

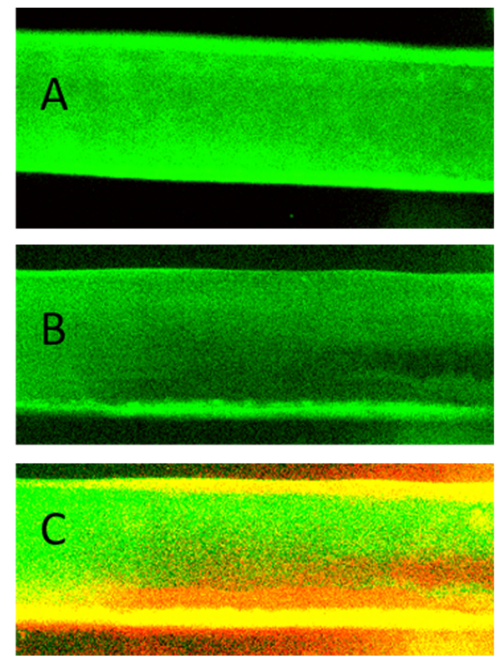

Figure 4. Stripping and backfilling lipids. A shows the initial bilayer (before stripping takes place). B shows the bilayer after stripping has taken place but before lipids have been backfilled. C shows the bilayer after stripping and backfilling. The lipids present initially were Fl-DOPE labeled (shown green) and the bilayer was backfilled with TR-DHPE labeled (shown red). Notice that the TR-DHPE lipids are most concentrated where the Fl-DOPE lipids have been stripped.

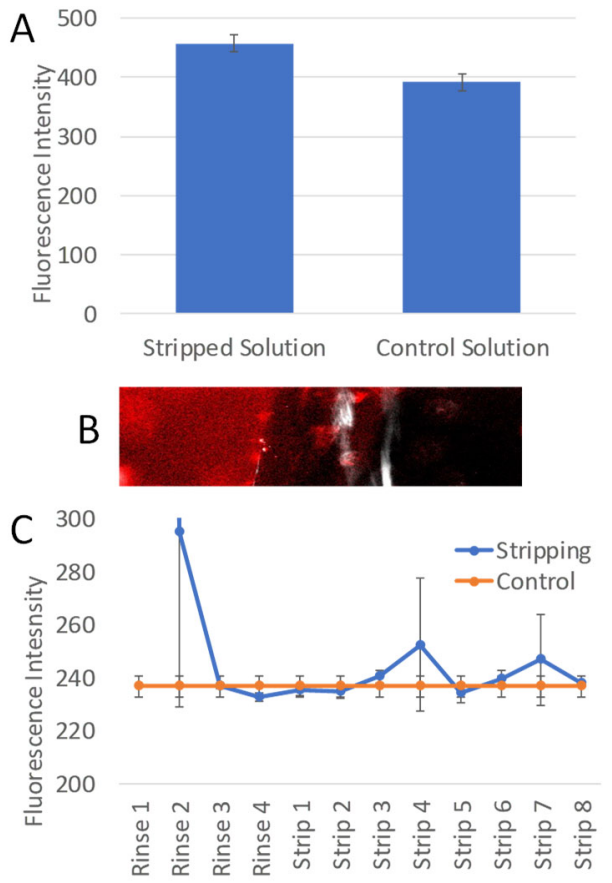

Figure 5. The fluorescence of the solution used to strip a bilayer from a solution compared to the fluorescence of a solution passed through the microfluidic device but with no fluorescent lipids present. A: the average fluorescence observed over 10 measurements for the stripped solution and the control solution. B: an image of the fluorescence observed with a fluorescence microscope of the stripped solution (left) a PDMS divider (center) and the control solution (right). C: observed fluorescence divided into time steps. The initial peak is due to unbound vesicles being rinsed away.

To verify that the lipids that were being removed were not simply migrating to other places in the device, the eluent used to strip a run was collected (not including the initial rinse, which was discarded) and compared to solution run through the device with no bilayer at identical flow rates and times. For each solution, around $0.02 \mathrm{~g}$ of SDS was added to the solution as it was being collected to ensure any lipids remained in solution. While no fluorescence was observed in either solution initially (which was expected, as the fluorescent lipids were highly diluted), upon concentration by evaporation (from $\sim 300 \mathrm{~mL}$ to $3 \mathrm{~mL}$ ) the stripped 
solution exhibited significantly greater fluorescence than the control solution (which was indistinguishable from noise, the difference was $>99.9 \%$ confidence level as determined by a t-test). These results are shown in Figure 5.

Finally, we feared that these results might be due to incomplete rinsing. To demonstrate that this was not the case, we collected the solution passed through the microfluidic device into aliquots of around $1.5 \mathrm{~mL}$ for rinsing and $30 \mathrm{~mL}$ for stripping. As before, around $0.02 \mathrm{~g}$ of SDS was added to each aliquot and then the aliquots were evaporated down to $1 \mathrm{~mL}$ and the fluorescence of each aliquot tested. The results are shown in Figure 5 C. As was expected, the fluorescence signal for the first two rinsing steps when lipids were used was much larger than any other signal (the first step fluorescence is so large that it is not shown, $>2000$ units), as in this step excess lipids (lipids that did not stick to the glass slide) were being rinsed away. By the third and fourth rinsing aliquots, the fluorescence signal was essentially indistinguishable from the control (which was noise-limited). When the flow rate was increased to induce stripping, the fluorescence was observed to increase with additional peaks in the fluorescence that at least roughly correlated to observed stripping events. It should be noted that this stripping occurred at different times in each run and Figure $5 \mathrm{C}$ shows the averaged results of three runs, thus none of the peaks shown are statistically different (as determined by a t-test, the greatest confidence level is at $70 \%$ ) than the control as a given peak typically only occurred in one of the three runs. However, on an individual run the difference between a peak and the control was significant $(>99 \%$ confidence level via t-test).

\section{DISCUSSION}

Others have previously obtained results suggesting that high velocity flow above a bilayer can cause changes to a bilayer, 41,42 and that lipid material can be removed from a bilayer during the formation of the bilayer, ${ }^{59}$ but to our knowledge this is the first report of high velocity flow moving SLB material into the aqueous solution without the aid of detergents. While our experiments were performed exclusively with lipids, this raises the possibility that such experiments could also be performed with membrane proteins contained in an SLB. This would be particularly valuable as a step following an SLB-based protein separation step, such as EOF, ${ }^{40}$ particularly if the removed SLB material forms vesicles as it is stripped from the surface. In lipid-only experiments, we attempted to determine the size of material coming off the supported lipid bilayer to determine if vesicle-like material or micellelike material was being generated, but the material generated was too dilute to be detected in our particle sizer.

In all experiments performed, it appeared that the stripping of materials from the support was not perfect - while significant sections of the stripped area have their lipids removed, small pockets of the original bilayer remain even in the stripped regions. In a set of stripping followed by backfilling experiments not shown, texas red-labeled lipid was stripped and the sections were backfilled with fluorescein (the reverse of the experiment shown in Figure 4). In this case, the stripping caused dark patches to appear, but when backfilling was attempted the increase in fluorescein fluorescence in the dark patch was relatively small. This is attributed to the FRET quenching of the fluorescein by remaining texas red-labeled lipid. This interpretation is also implied by the fact that only very rarely do the stripped regions get as dark as the lipid-free regions (e.g. in Figure 2, compare the stripped regions to the regions with no bilayer due to PDMS protection).

\section{CONCLUSIONS}

We have demonstrated material can be removed from a supported lipid bilayer by high-speed buffer flow over the bilayer. This was accomplished inside of a microfluidic device by flowing solution over the SLB at flow rates of from 1 to around $4 \mathrm{~m} / \mathrm{s}$ linear flow rate. Stripping of material from the supported lipid bilayer was observed to take place at flow rates greater than $1 \mathrm{~m} / \mathrm{s}$ and with all aqueous solutions tested. Stripping was demonstrated both by observing lipid loss from the bilayer using fluorescence microscopy and by monitoring fluorescence in the solution used to strip the material from the bilayer. Our results suggest that, if previously developed bilayer-based techniques were used to purify proteins in an SLB, ${ }^{38-45}$ the method we report in this paper could then be used to generate solution-phase purified proteins and lipids. This could be valuable for further biochemical work, and we are developing a device to attempt this. Alternatively, stripping SLBs using high speed buffer flow could be used to generate SLBs with compositionally distinct regions.

\section{ACKNOWLEDGEMENTS}

The authors thank Professor Susan Harris for making the ceramic rack used to anneal our glass coverslips. We thank Joshua A. Reid and Whitney N. Hansen for their work on this project. This project was funded by Southern Utah University's Faculty Scholarly Support Fund, undergraduate research grants, and Skaggs endowment grants. C. C., M. V., and S. S. wish to thank the Walter Maxwell Gibson endowment for research fellowships.

\section{REFERENCES}

1. Johnson, S. J., Bayerl, T. M., McDermott, D. C., Adam, G. W., Rennie, A. R., Thomas, R. K., Sackmann, E. (1991) Structure of an Adsorbed Dimyristoylphosphatidylcholine Bilayer Measured with Specular Reflection of Neutrons, Biophys J 59, 289294. https:/ / doi.org/ 10.1016/S0006-3495(91)82222-6 
2. Tamm, L. K., McConnell, H. M. (1985) Supported Phospholipid Bilayers, Biophys J 47, 105-113. https:// doi.org/ 10.1016/S0006-3495(85)83882-0

3. Smith, B. A., McConnell, H. M. (1978) Determination of Molecular Motion in Membranes Using Periodic Pattern Photobleaching, Proc Natl Acad Sci USA 75, 2759-2763. https:/ / doi.org/10.1073/pnas.75.6.2759

4. Kurniawan, J., Ventrici de Souza, J. F., Dang, A. T., Liu, G.-Y., Kuhl, T. L. (2018) Preparation and Characterization of SolidSupported Lipid Bilayers Formed by Langmuir-Blodgett Deposition: A Tutorial, Langmuir 34, 15622-15639. https:// doi.org/10.1021/acs.langmuir.8b03504

5. Castellana, E. T., Cremer, P. S. (2006) Solid Supported Lipid Bilayers: From Biophysical Studies to Sensor Design, Surf Sci Rep 61, 429-444. https:/ / doi.org/10.1016/j.surfrep.2006.06.001

6. Phizicky, E., Bastiaens, P. I. H., Zhu, H., Snyder, M., Fields, S. (2003) Protein analysis on a proteomic scale, Nature 422, 208215. https://doi.org/10.1038/ nature01512

7. Zhu, H., Bilgin, M., Bangham, R., Hall, D., Casamayor, A., Bertone, P., Lan, N., Jansen, R., Bidlingmaier, S., Houfek, T., Mitchell, T., Miller, P., Dean, R. A., Gerstein, M., Snyder, M. (2001) Global analysis of protein activities using proteome chips, Science 293, 2101-2105. https:/ / doi.org/10.1126/science.1062191

8. Sendecki, A. M., Poyton, M. F., Baxter, A. J., Yang, T., Cremer, P. S. (2017) Supported Lipid Bilayers with Phosphatidylethanolamine as the Major Component, Langmuir 33, 13423-13429. https:// doi.org/ 10.1021/acs.langmuir. 7 b02323

9. Veatch, S. L., Keller, S. L. (2005) Seeing Spots: Complex Phase Behavior in Simple Membranes, Biochim Biophys Acta 1746, 172-185. https://doi.org/10.1016/j.bbamcr.2005.06.010

10. Rayermann, S. P., Rayermann, G. E., Cornell, C. E., Merz, A. J., Keller, S. L. (2017) Hallmarks of Reversible Separation of Living, Unperturbed Cell Membranes into Two Liquid Phases, Biophys J 113, 2425-2432. bttps:/ / doi.org/10.1016/j.bpj.2017.09.029

11. Tabaei, S. R., Jackman, J. A., Kim, S.-O., Liedberg, B., Knoll, W., Parikh, A. N., Cho, N.-J. (2014) Formation of CholesterolRich Supported Membranes Using Solvent-Assisted Lipid Self-Assembly, Langmuir 30, 13345-13352. https:// doi.org/10.1021/la5034433

12. Mossman, K. D., Campi, G., Groves, J. T., Dustin, M. L. (2005) Altered TCR Signaling from Geometrically Repatterned Immunological Synapses, Science 310, 1191-1193. https:/ / doi.org/10.1126/ science.1119238

13. Kung, L. A., Kam, L., Hovis, J. S., Boxer, S. G. (2007) Patterning Hybrid Surfaces of Proteins and Supported Lipid Bilayers, Langmuir 16, 6773-6776. bttps:/ / doi.org/10.1021/la000653t

14. Nair, P. M., Salaita, K., Petit, R. S., Groves, J. T. (2011) Using Patterned Supported Lipid Membranes to Investigate the Role of Receptor Organization in Intracellular Signaling, Nat Protoc 6, 523-539. https:// doi.org/ 10.1038/nprot.2011.302

15. Mossman, K., Groves J. (2007) Micropatterned Supported Membranes as Tools for Quantitative Studies of the Immunological Synapse, Chem Soc Rev 36, 46-54. https:// doi.org/10.1039/b605319j

16. Nabika, H., Sasaki, A., Takimoto, B., Sawai, Y., He, S., Murakoshi, K. (2005) Controlling Molecular Diffusion in SelfSpreading Lipid Bilayer Using Periodic Array of Ultra-Small Metallic Architecture on Solid Surface J Am Chem Soc 127, 16786-16787. https://doi.org/10.1021/ja0559597

17. Hughes, L. D., Boxer, S. G. (2013) DNA-Based Patterning of Tethered Membrane Patches, Langmuir 29, $12220-12227$. bttps://doi.org/10.1021/la402537p

18. Cremer, P. S., Boxer, S. G. (1999) Formation and Spreading of Lipid Bilayers on Planar Glass Supports, J Phys Chem 103, 2554-2559. https://doi.org/10.1021/jp983996x

19. Groves, J. T., Boxer, S. G. (1995) Electric Field-Induced Concentration Gradients in Planar Supported Bilayers, Biophys J 69, 1972-1975. https://doi.org/10.1016/S0006-3495(95)80067-6

20. Groves, J. T., Wülfing, C., Boxer, S. G. (1996) Electrical Manipulation of Glycan-Phosphatidyl Inositol-Tethered Proteins in Planar Supported Bilayers, Biophys J 71, 2716-2723. https:/ / doi.org/10.1016/S0006-3495(96)79462-6

21. Groves, J. T., Boxer, S. G., McConnell, H. M. (1997) Electric field-induced reorganization of two-component supported bilayer membranes, Proc Natl Acad Sci USA 94, 13390-13395. bttps:/ / doi.org/10.1073/pnas.94.25.13390

22. Grogan M. J., Kaizuka, Y., Conrad, R. M., Groves, J. T., Bertozzi, C. R. (2005) Synthesis of Lipidated Green Fluorescent Protein and its Incorporation in Supported Lipid Bilayers, J Am Chem Soc 127, 14383-14387. https://doi.org/10.1021/ja052407f

23. Bulloch, T. M., Argyle, T. C., Parson, M. G., Monson, C. F. (2016) Patterning Supported Lipid Bilayers Using Magnetic Tweezers, J Utah Acad 93, 269-281.

24. Zhu, Y., Moran-Mirabal, J. (2016) Micropatterning of Phase-Segregated Supported Lipid Bilayers and Binary Lipid Phases through Polymer Stencil Lift-Off, Langmuir 32, 11021-11028. https:/ / doi.org/ 10.1021/ acs.langmuir.6b02346

25. Richards, M. J., Daniel, S. (2018) Two-Phase Contiguous Supported Lipid Bilayer Model for Membrane Rafts via Polymer Blotting and Stenciling, Langmuir 33, 1285-1294. https:/ / doi.org/10.1021/ acs.langmuir.6b04385

26. Zhou, Y., Gao, J., Zhu, H., Xu, J., He, H., Gu, L., Wang, H., Chen, J., Ma, D., Zhou, H., Zheng, J. (2018) Enhancing Membrane Protein Identification Using a Simplified Centrifugation and Detergent-Based Membrane Extraction Approach, Anal Chem 90, 2434-2439. https:/ / doi.org/10.1021/ acs.analchem. 7603710 
27. Santoni V., Molloy, M., Rabilloud, T. (2000) Membrane proteins and proteomics: Un amour impossible? Electrophor 21, 10541070. bttps:/ / doi.org/ 10.1002/(SICI)1522-2683(20000401)21:6<1054::AID-ELPS1054>3.0.CO;2-8

28. Braun R. J., Kinkl, N., Beer, M., Ueffing, M. (2007) Two-dimensional electrophoresis of membrane proteins, Anal Bioanal Chem 389, 1033-1045. https:// doi.org/10.1007/s00216-007-1514-6

29. Rabilloud T. (2009) Membrane proteins and proteomics: love is possible, but so difficult, Electrophor 30 (S1), S174-S180. https:/ / doi.org/ 10.1002/ elps.200900050

30. Berweger, S., Nguyen, D. M., Muller, E. A., Bechtel, H. A., Perkins, T. T., Raschke, M. B. (2013) Nano-Chemical Infrared Imaging of Membrane Proteins in Lipid Bilayers J Am Chem Soc 135, 18292-18295. https://doi.org/10.1021/ja409815g

31. Long, F., Su, C. C., Zimmermann, M. T., Boyken, S. E., Rajashankar, K. R., Jernigan, R. L., Yu, E. W. (2010) Crystal Structures of the CusA Efflux Pump Suggest Methionine-Mediated Metal Transport, Nature 467, 484-488. https:// doi.org/10.1038/ nature09395

32. Sharma, V. K., Qian, S. (2019) Effect of an Antimicrobial Peptide on Lateral Segregation of Lipids: A Structure and Dynamics Study by Neutron Scattering, Langmuir 35, 4152-4160. https:/ / doi.org/ 10.1021/ acs.langmuir.8b04158

33. Pace, H., Nyström L. S., Gunnarsson, A., Eck, E., Monson, C., Geschwindner, S., Snijder, A., Höök, F. (2015) Preserved Transmembrane Protein Mobility in Polymer-Supported Lipid Bilayers Derived from Cell Membranes, Anal Chem 87, $9194-$ 9203. https://doi.org/10.1021/acs.analchem.5b01449

34. Davis, R. W., Flores, A., Barrick, T. A., Cox, J. M., Brozik, S. M., Lopez, G. P., Brozik, J. A. (2007) Nanoporous Microbead Supported Bilayers: Stability, Physical Characterization, and Incorporation of Functional Transmembrane Proteins, Langmuir 23, 3864-3872. https:// doi.org/10.1021/la062576t

35. Zieleniecki, J. L., Nagarajan, Y., Waters, S., Rongala, J., Thompson, V., Hrmova, M., Köper, I. (2016) Cell-Free Synthesis of a Functional Membrane Transporter into a Tethered Bilayer Lipid Membrane, Langmuir 32, 2445-2449. bttps:/ / doi.org/10.1021/ acs.langmuir.5b04059

36. Casiraghi, M., Damian, M., Lescop, E., Point, E., Moncoq, K., Morellet, N., Levy, D., Marie, J., Guittet, E., Banères, J.-L., Catoire, L. J. (2016) Functional Modulation of a G Protein-Coupled Receptor Conformational Landscape in a Lipid Bilayer, J Am Chem Soc 138, 11170-11175. https://doi.org/10.1021/jacs.6b04432

37. Daniel, S., Diaz, A. J., Martinez, K. M., Bench, B. J., Albertorio, F., Cremer, P. S. (2007) Separation of Membrane-Bound Compounds by Solid-Supported Bilayer Electrophoresis, J Am Chem Soc 129, 8072-8073. https://doi.org/10.1021/ja0720816

38. Tanaka, M., Hermann, J., Haase, I., Fischer, M., Boxer, S. G. (2007) Frictional Drag and Electrical Manipulation of Recombinant Proteins in Polymer-Supported Membranes, Langmuir 23, 5638-5644. https:/ / doi.org/10.1021/la0628219

39. Monson, C. F., Pace, H. P., Liu, C., Cremer, P. S. (2011) Supported Bilayer Electrophoresis under Controlled Buffer Conditions, Anal Chem 83, 2092-2096. https://doi.org/10.1021/ac1028819

40. Liu, C., Monson, C. F., Yang, T., Pace, H., Cremer, P. S. (2011) Protein Separation by Electrophoretic-Electroosmotic Focusing on Supported Lipid Bilayers, Anal Chem 83, 7876-7880. https:// doi.org/10.1021/ac201768k

41. Jönsson, P., Beech, J. P., Tegenfeldt, J. O., Höök, F. (2009) Shear-Driven Motion of Supported Lipid Bilayers in Microfluidic Channels, J Am Chem Soc 131, 5294-5297. https:/ / doi.org/10.1021/ja809987b

42. Jönsson, P., Beech, J. P., Tegenfeldt, J. O., Höök, F. (2009) Mechanical Behavior of a Supported Lipid Bilayer under External Shear Forces, Langmuir 25, 6279-6286. https://doi.org/10.1021/la8042268

43. Hoopes, M. I., Faller, R., Longo, M. L. (2011) Lipid Domain Depletion at Small Localized Bends Imposed by a Step Geometry, Langmuir 27, 2783-2788. https://doi.org/10.1021/la104504p

44. Motegi, T., Nabika, H., Fu, Y., Chen, L., Sun, Y., Zhao, J., Murakoshi, K. (2014) Effective Brownian Ratchet Separation by a Combination of Molecular Filtering and a Self-Spreading Lipid Bilayer System, Langmuir 30, 7496-7501. bttps:// doi.org/10.1021/la500943k

45. Neumann, J., Hennig, M., Wixforth, A., Manus, S., Rädler, J. O., Schneider, M. F. (2010) Transport, Separation, and Accumulation of Proteins on Supported Lipid Bilayer, Nano Lett 10, 2903-2908. https://doi.org/10.1021/nl100993r

46. Wagner, M. L., Tamm, L. K. (2000) Tethered Polymer-Supported Planar Lipid Bilayers for Reconstitution of Integral Membrane Proteins: Silane-Polyethyleneglycol-Lipid as a Cushion and Covalent Linker, Biophys J 79, $1400-1414$. bttps:/ / doi.org/10.1016/S0006-3495(00)76392-2

47. Wagner, M. L., Tamm, L. K. (2001) Reconstituted Syntaxin1A/SNAP25 Interacts with Negatively Charged Lipids as Measured by Lateral Diffusion in Planar Supported Bilayers, Biophys J 81, 266-275. https:/ / doi.org/10.1016/S00063495(01)75697-4

48. Goennenwein, S., Tanaka, M., Hu, B., Moroder, L., Sackmann, E. (2003) Functional Incorporation of Integrins into Solid Supported Membranes on Ultrathin Films of Cellulose: Impact on Adhesion, Biophys J 85, 646-655. bttps:/ / doi.org/10.1016/S0006-3495(03)74508-1

49. Purrucker, O., Förtig, A., Jordan, R., Tanaka, M. (2004) Supported Membranes with Well-Defined Polymer TethersIncorporation of Cell Receptors, ChemPhysChem 5, 327-335. https:/ / doi.org/ 10.1002/ pphc.200300863 
50. Purrucker, O., Förtig, A., Jordan, R., Sackmann, E., Tanaka, M. (2007) Control of Frictional Coupling of Transmembrane Cell Receptors in Model Cell Membrnes with Linear Polymer Spacers, Phys Rev Lett 98, 078102. bttps:// doi.org/10.1103/PhysRevLett.98.078102

51. Richards, M. J., Hsia, C.-Y., Singh, R. R., Haider, H., Kumpf, J., Kawate, T., Daniel, S. (2016) Membrane Protein Mobility and Orientation Preserved in Supported Bilayers Created Directly from Cell Plasma Membrane Blebs, Langmuir 32, 29632974. bttps:/ / doi.org/10.1021/acs.langmuir.5b03415

52. Hertrich, S., Stetter, F., Rühm, A., Hugel, T., Nickel, B. (2014) Highly Hydrated Deformable Polyethylene Glycol-Tethered Lipid Bilayers, Langmuir 30, 9442-9447. https:/ / doi.org/10.1021/ la4045804

53. Kam, L., Boxer, S. G. (2003) Spatially Selective Manipulation of Supported Lipid Bilayers by Laminar Flow: Steps Toward Biomembrane Microfluidics, Langmuir 19, 1624-1631. bttps:/ / doi.org/ 10.1021 / la0263413

54. Lee, S. C., Knowles, T. J., Postis, V. L. G., Jamshad, M., Parslow, R. A., Lin, Y., Goldman, A., Sridhar, P., Overduin, M., Muench, S. P., Dafforn, T. (2016) A Method for Detergent-free isolation of Membrane Protein from its Local Lipid Environment, Nature Prot 11, 1149-1162. https:/ / doi.org/ 10.1038/ nprot.2016.070

55. Simon, K. S., Pollock, N. L., Lee, S. C. (2018) Membrane protein nanoparticles: the shape of things to come, Biochem Soc Trans 46, 1495-1504. https:// doi.org/10.1042/BST20180139

56. Hope, M. J., Bally, M. B., Webb, G., Cullis, P. R. (1985) Production of Large Unilamellar Vesicles by a Rapid Extrusion Procedure: Characterization of Size Distribution, Trapped Volume and Ability to Maintain a Membrane Potential, Biochim Biophys Acta 812, 55-65. https:// doi.org/10.1016/0005-2736(85)90521-8

57. Mayer, L. D., Hope, M. J., Cullis, P. R. (1986) Vesicles of Variable Sizes Produced by a Rapid Extrusion Procedure, Biochim Biophys Acta 858, 161-168. https:/ / doi.org/10.1016/0005-2736(86)90302-0

58. Yang, T., Jung, S.-Y., Mao, H., Cremer, P. S. (2001) Fabrication of Phospholipid Bilayer-Coated Microchannels for On-Chip Immunoassays, Anal Chem 73, 165-169. https:// doi.org/10.1021/ac000997o

59. Zhu, L., Gregurec, D., Reviakine, I. (2013) Nanoscale Departures: Excess Lipid Leaving the Surface during Supported Lipid Bilayer Formation, Langmuir 29, 15283-15292. https:/ / doi.org/10.1021/la401354j

60. Pace, H. P., Sherrod, S. D., Monson, C. F., Russell, D. H., Cremer, P. S. (2013) Coupling Supported Lipid Bilayer Electrophoresis with MALDI-MS Imaging, Anal Chem 85, 6047-6052. bttps:/ / doi.org/10.1021/ac4008804

\section{ABOUT STUDENT AUTHORS}

Michael J. Ornstead graduated in April 2019 and is currently working on his MD at the University of Utah.

Ruth Hunter graduated in April 2020 and is planning to attend medical school.

Mason L. Valentine graduated in April 2015 and is getting a PhD at the University of Texas, Austin.

Cameron Cooper graduated in April 2015, earned a degree as a physician assistant, and is currently working in the field.

Stephen K. Smith graduated in April 2019 and is currently working on a dental degree at the University of Utah.

\section{PRESS SUMMARY}

Cell membranes, the "bags" that hold our cells together, are composed of lipids and membrane proteins. Membranes are essential parts of cells, with many different processes occurring at or across them. Membranes are also difficult to study biochemically, as they have both water and oil soluble parts. Because of this, several methods to study membranes are being developed around the world. Many of them use supported lipid bilayers, flat sheets of membrane material sitting on a solid support such as glass. The authors have developed a method to study lipids in a supported lipid bilayer from the glass and put the material into solution. This is accomplished by flowing solution over the lipid sheet at high speeds. This could help with efforts to learn more about membranes, particularly membrane proteins, as it may allow supported lipid bilayers to be converted into more cell-like structures. 


\title{
Assessing Initiatives for Rural Health Practices in South Carolina
}

\author{
Aalia Soherwardy ${ }^{* a}$ \& Elizabeth Crouch $^{b}$ \\ ${ }^{a}$ University of South Carolina School of Medicine, Columbia, SC \\ ${ }^{b}$ Rural and Minority Health Research Center, Arnold School of Public Health, University of South Carolina, Columbia, South Carolina
}

bttps:// doi.org/10.33697/ajur.2020.028

Student:aalia@email.sc.edu

Mentor: crouchel@mailbox.sc.edu*

\begin{abstract}
The purpose of this study was to determine which incentives are most effective in motivating medical students to practice in rural areas of South Carolina, which can be informative for the medical practitioner rural recruitment process. Medical students attending the University of South Carolina School of Medicine located in Columbia, South Carolina were surveyed about demographic information, motivations for rural practice, and considerations for choosing a practice location (n=109). Chi-square tests and bivariate analyses were used to test for significant differences. A significant relationship was found between previous residence in a rural area and personal motivation to practice in a rural area $(\mathrm{p}<0.001)$. It was also found that $86.2 \%$ of students who had previously lived, worked, or served in rural areas had a personal motivation to practice medicine in a rural area, confirming previous research. Loan forgiveness options were the most appealing personal incentive for the students in this study, closely followed by guaranteed minimum incomes and tax incentives; financial incentives were more preferred than non-financial incentives like reduced on-call work and accelerated residencies. The results of this study can be utilized to craft future statesupported incentive programs or to tailor current programs to more effectively recruit students to rural practice.
\end{abstract}

\section{KEY WORDS}

Rural; Recruitment; Healthcare Provider; Shortage; Incentive Programs; Medical Student; Southern United States; Loan Forgiveness

\section{INTRODUCTION}

More than one in every ten Americans lives in health professional shortage areas, with limited to access to healthcare and healthcare professionals. ${ }^{1}$ Healthcare professional shortage areas are predominantly located in rural areas because they have difficulties in recruiting and retaining enough healthcare providers. ${ }^{1}$ This shortage of physicians and healthcare providers creates healthcare access issues for rural residents. ${ }^{2}$

Yet, rural areas are more likely to be health professional shortage areas, despite programs aiming to incentive clinical practice in rural areas. ${ }^{1}$ In an effort to overcome this healthcare provider shortage, numerous incentive programs have been established, both in the U.S. and internationally, in an attempt to attract new health professional graduates towards rural communities. ${ }^{1}$ In 2008 , for example, there were 75 state-supported incentive programs operating around the United States that rewarded healthcare professionals for working in underserved areas. ${ }^{3}$

Previous research found that two-thirds of general practitioners who had recently graduated would consider rural areas for their practices if an appropriate incentive scheme were available. ${ }^{4}$ The strongest incentive factors included guaranteed minimum income, loan forgiveness options, and family considerations. ${ }^{4}$ Financial and lifestyle accommodations have also been found to be critical in motivating students to choose rural over metropolitan practice sites. ${ }^{5}$ Finally, a rural background or connection has been related to high recruitment and retention rates of physicians. ${ }^{1}$

There have been efforts to incentivize rural practice, but the evidence around their effectiveness is limited. Previous literature has found that loan forgiveness-based programs appear to support healthcare provider recruitment for rural practice. ${ }^{1}$ However, it is unclear how incentive schemes directly affect the rural workforce in a community. ${ }^{2}$ A 2000 report from the World Health Organization found that financial-based incentive programs have had very little influence on the geographic distribution of health professionals on an international level. ${ }^{2}$ Recruiters and students in previous literature considered lifestyle considerations to be more important than financial incentives. ${ }^{4}$ Therefore, it may be difficult for rural communities to overcome the obstacles they face in attracting doctors by using financial incentives alone. ${ }^{6}$ 
It is important to increase the number of providers in rural areas because rural areas generally have worse health outcomes. ${ }^{8}$ This is especially true in the South. ${ }^{8}$ Rural areas rank poorly in numerous population health indicators such as health behaviors, mortality, morbidity, and maternal and child health measures. ${ }^{8}$ Particularly in the American South, rural residents face higher rates of poverty, adult smoking, physical inactivity, ischemic heart disease-related deaths, and births to adolescents. ${ }^{8}$ The heart disease mortality rate was highest in the rural South and $25 \%$ higher than the mortality rate of Southerners in suburban settings. ${ }^{9}$ Residents of rural counties in the South also have higher infant mortality rates and higher blood pressure compared to residents of metropolitan and suburban counties. ${ }^{9}$ Provider coverage also varies, with rural physicians less likely to be specialists, and rural hospitals more likely to have a limited range of care for patients. ${ }^{10}$ These disparities make it more difficult to redress the health problems experienced in the rural South.

South Carolina is a state with a relatively large rural population. ${ }^{11}$ Many of the rural areas in South Carolina are also health professional shortage areas and have poorer health outcomes than urban areas of the state. ${ }^{12}$ The need for better access to healthcare is especially great for those living in the rural areas of South Carolina. In this state, 29 out of 46 counties are considered nonmetropolitan areas and nearly $14 \%$ of the population resides in poverty. ${ }^{11}$ Nearly a quarter of South Carolinians reside in rural counties, but only $10 \%$ of South Carolina physicians have established their main practices in rural counties. ${ }^{12}$ South Carolina has a higher proportion of African-American residents and rural residents than national averages, but a lower proportion of residents with a bachelor's degree or higher. ${ }^{11}$ These characteristics are also representative of many rural Southern states.

Therefore, understanding which incentives may motivate current medical students in South Carolina to practice in rural areas is important for betting understanding how to solve the problem of rural provider shortages in South Carolina. Most rural practice incentive schemes are not formulated based on research regarding the target populations of the incentive programs. If a program can be tailored to which incentives are known to attract individuals currently studying medicine, it would likely have a much higher chance of success in recruiting more medical practitioners to practice in a rural setting. Therefore, the purpose of this study is to assess which incentives may be the strongest factors to motivate medical students to practice in rural areas of South Carolina. The study also seeks insight into intrinsic versus extrinsic motivations in medical students.

\section{METHODS AND PROCEDURES}

\section{Participants}

The survey sample targeted all medical students attending the University of South Carolina School of Medicine located in Columbia, South Carolina. All students who had been enrolled as first-year, second-year, third-year, or fourth-year medical students at the time of survey dissemination were invited to participate. The total number of included responses in the sample was 109 out of the population of 373 professional students enrolled at the School of Medicine.

\section{Instrument}

The same survey was administered to all participants through SurveyMonkey (January - March 2018) with the electronic link provided in the letter of invitation (Appendix A). The survey contained 16 questions divided into three sections: demographic information, motivations for rural practice, and considerations for choosing a practice location. Before a participant could begin, they were instructed to consider a rural area to be any area within South Carolina not considered inside the major metropolitan areas of Greenville, Columbia, and Charleston for the purposes of the research survey.

The first section of the survey, demographic information, inquired about the participant's gender, age, race/ethnicity, relationship status, and level of medical education. Age was categorized into the following groups: "17 or younger", "18-20", "21-29", "3039", "40-49", "50-59", and "60 or older". Race/ethnicity included American Indian or Alaskan Native, Asian or Pacific Islander, Black or African American, Hispanic or Latino, White/Caucasian, and Other, with an option to not indicate race. Relationship status was categorized into the following groups: married; widowed; divorced; separated; single, but cohabiting with a significant other; and single, never married. Level of medical education was divided into four response categories: first-year, second-year, third-year, and fourth-year medical student.

Additional survey questions were included to understand and examine the willingness of a medical student to work in a rural area after graduation. The first question asked about the total amount of money owed in student loans by the time of graduation, with five response categories: “ $<\$ 50,000 ”, " \$ 50,000$ - $\$ 100,000 ”, " \$ 100,000$ - $\$ 150,000 ”, " \$ 150,000$ - $\$ 200,000 ”$, and “ $>\$ 200,000 ”$. This question was asked as financial obligations may entice students to work for loan forgiveness. ${ }^{1}$ Another survey item asked the respondent if they had ever lived, worked, or served in a rural area. This item was important to include as rural exposure has previously been associated with willingness to work in a remote setting, ${ }^{13}$ and personal rural connections are associated with high recruitment and retention. ${ }^{1}$ Further survey items in this section inquired about a student's interest in primary care and preferred medical specialties of the respondents, with response categories limited to internal medicine, obstetrics/gynecology, pediatrics, family medicine, and the option to specify other specialties. 
The next section, motivations for rural practice, contained only two items. The first question asked if the respondent had any personal motivations to practice medicine in a rural area. If they answered yes, they were asked to specify their personal incentives for rural practice and then skipped the next item via skip logic built into the electronic survey. If the respondent indicated "no" or "unsure," they proceeded to the second item in this section that asked if they would be interested in rural practice if sufficiently incentivized. Some individuals have intrinsic motivations that would lead them to practice in a remote area without compensation, such as a philanthropic nature. ${ }^{14}$ However, it may be unrealistic to expect physicians to practice in a rural setting without any financial incentives. ${ }^{5}$

The final section, considerations for choosing a practice location, asked respondents to indicate which of the fourteen factors they deemed important when considering a practice location in two survey items. Items fall in the themes of community characteristics (income potential and types of recreational activities available), professional development (career advancement and experience opportunities), family influences (influence of spouse/partner and quality of education), and education-related considerations (financial aid and previous training). ${ }^{1}$ The list included factors such as community need, financial aid obligations, community size, a desire to return to hometown, and area-specific program participation. These factors were included in previous studies and were highly rated by health professionals inclined to practice in a rural area. ${ }^{1}$ The respondents were presented with these 14 factors in two survey items structured in identical formats asking of the practice location in a rural setting in one survey item and in a nonrural setting in the second.

The survey then asked the respondent to rate a list of structural incentives statements, such as access to a high-complexity regional hospital, on a five-point scale from "strongly disagree" to "strongly agree." 15 Items in this section included a question that was infrastructure-based, such as practicing in an accessible geographic area, as well as a question examining access to online information technology. ${ }^{13}$ The survey also asked the respondent to rate a list of financial incentives, such as loan forgiveness options, on the same five-point scale. This question allows respondents to directly compare monetary compensations and lifestyle considerations. The last item of the section and the survey asked how much loan forgiveness the respondent would consider to be a reasonable incentive. The question has five response categories: “<\$50,000”, “\$50,000 - \$100,000”, “\$100,000 - $\$ 150,000 "$, “\$150,000 - \$200,000", and ">\$200,000". A short conclusion section to the survey followed, thanking the respondent and providing a contact for further questions.

\section{Survey Administration}

The Institutional Review Board approved the study (Approval Number Pro00073259). All data collected was anonymous, and none of the responses submitted to the SurveyMonkey platform contained any identifying information. Participants were informed of the purpose and requirements of the study prior to accessing the survey, where they must agree to continue based on the information they are given about the study. Participants could withdraw participation at any time during the survey with no consequences. No compensation was provided to participants.

A letter of invitation was disseminated to all medical students through the email of the Associate Dean for Medical Education and Academic Affairs at the University of South Carolina School of Medicine. Some students were also asked to participate in the survey and provided an electronic link to the survey in medical school classrooms with permission of the instructor.

All survey responses were checked for inconsistent data, missing demographic data, and outliers. Missing or incomplete responses $(n=2)$ were omitted from the data pool utilized for analysis.

\section{Analysis}

Descriptive statistics were calculated and chi-square tests were performed, using Excel.

\section{RESULTS}

The majority of the sample was male (52.3\%), 21-29 years old (90.8\%), white/Caucasian (86.2\%), and had never married (61.4\%; Table 1). The predominant group of survey participants was composed of second-year and third-year medical students. Most respondents owed at least $\$ 100,000$ in student loans $(67 \%)$ and had lived, worked, or served in a rural area $(52.3 \%)$, and were interested in primary care $(45.9 \%)$. Internal medicine was the medical specialty of most interest $(16.5 \%)$. The majority of the students entered "Other" for the medical specialty of interest. Most respondents did not have personal motivations to practice in a rural area or were unsure $(72.4 \%$, Table 2$)$. If sufficiently incentivized, the majority of respondents without intrinsic motivations would consider rural practice (Table 2 ). 


\begin{tabular}{|c|c|c|}
\hline Factor & $\mathrm{N}=109$ & Percentage \\
\hline \multicolumn{3}{|l|}{ Gender } \\
\hline Male & 57 & $52.3 \%$ \\
\hline Female & 49 & $45.0 \%$ \\
\hline \multicolumn{3}{|l|}{ Age } \\
\hline $18-20$ & 1 & $0.92 \%$ \\
\hline $21-29$ & 99 & $90.8 \%$ \\
\hline $30-39$ & 7 & $6.4 \%$ \\
\hline \multicolumn{3}{|l|}{ Ethnicity } \\
\hline White/Caucasian & 94 & $86.2 \%$ \\
\hline Asian or Pacific Islander & 7 & $6.4 \%$ \\
\hline Black/African American & 3 & $2.7 \%$ \\
\hline Other & 3 & $2.7 \%$ \\
\hline \multicolumn{3}{|l|}{ Relationship Status } \\
\hline Single, never married & 67 & $61.4 \%$ \\
\hline Single, but cohabiting & 21 & $19.3 \%$ \\
\hline Married & 18 & $16.5 \%$ \\
\hline Separated & 1 & $0.92 \%$ \\
\hline \multicolumn{3}{|c|}{ Medical Education Level } \\
\hline First-year & 24 & $22.0 \%$ \\
\hline Second-year & 30 & $27.5 \%$ \\
\hline Third-year & 34 & $31.2 \%$ \\
\hline Fourth-year & 20 & $18.3 \%$ \\
\hline \multicolumn{3}{|c|}{ Total Amount Owed in Student Loans by time of graduation } \\
\hline$<\$ 50,000$ & 11 & $10.1 \%$ \\
\hline$\$ 50,000-\$ 100,000$ & 23 & $21.1 \%$ \\
\hline$\$ 100,000-\$ 150,000$ & 27 & $24.8 \%$ \\
\hline$\$ 150,000-\$ 200,000$ & 21 & $19.3 \%$ \\
\hline$>\$ 200,000$ & 25 & $22.9 \%$ \\
\hline \multicolumn{3}{|c|}{ Lived, Worked, Served in Rural Area, by self-report } \\
\hline Yes & 57 & $52.3 \%$ \\
\hline No & 51 & $46.8 \%$ \\
\hline \multicolumn{3}{|l|}{ Interest in Primary Care } \\
\hline No & 57 & $52.3 \%$ \\
\hline Yes & 50 & $45.9 \%$ \\
\hline \multicolumn{3}{|c|}{ Medical Specialties of Interest } \\
\hline Internal Medicine & 18 & $16.5 \%$ \\
\hline Family Medicine & 14 & $12.8 \%$ \\
\hline Pediatrics & 10 & $9.2 \%$ \\
\hline Obstetrics/Gynecology & 9 & $8.3 \%$ \\
\hline Other & 56 & $51.4 \%$ \\
\hline
\end{tabular}

Table 1. Demographic Information

\begin{tabular}{|l|l|l|l|l|l|}
\hline $\begin{array}{l}\text { Personal } \\
\text { Motivations for } \\
\text { Rural Practice }\end{array}$ & $\mathbf{N}$ & Percentage & $\begin{array}{l}\text { If Sufficiently } \\
\text { Incentivized... }\end{array}$ & N & Percentage \\
\hline No & 43 & $39.4 \%$ & No & 8 & $10.1 \%$ \\
\hline Unsure & 36 & $33.0 \%$ & Unsure & 26 & $32.9 \%$ \\
\hline Yes & 30 & $27.5 \%$ & Yes & 45 & $57.0 \%$ \\
\hline
\end{tabular}

Table 2. Motivations for Rural Practice \& Incentive Interest ( $N=109)$

Out of the 14 factors listed, the primary factors for considering rural practice were income potential (76.1\%), serving the health needs of the community (70.6\%), regional and recreational activities available $(67.9 \%)$, quality of education for child(ren) (66.1\%), and proximity to extended family/relatives $(61.5 \%$, Table 3$)$. Out of the 14 factors listed for considering a rural practice, 
respondents considered location of previous clinical training/residency $(13.8 \%)$ and participation in area-specific training program $(12.8 \%)$ to be the least important factors (Table 3). Factors considered by students for not practicing in a rural location included the quality of education for child(ren) $(79.8 \%)$, income potential $(76.1 \%)$, regional and recreational activities available $(76.1 \%)$, opportunity for career advancement (75.2\%), and influence of spouse/partner $(71.6 \%$, Table 4$)$. The lowest factors under consideration by survey respondents were previous clinical training/residency $(26.6 \%)$ and return to hometown $(18.3 \%)$.

\begin{tabular}{|l|l|l|}
\hline Factor & N & Percentage \\
\hline Income Potential & 83 & $76.1 \%$ \\
\hline Serving the Health Needs of the Community & 77 & $70.6 \%$ \\
\hline Influence of Spouse/Partner & 66 & $60.6 \%$ \\
\hline Quality of Education for Child(ren) & 72 & $66.1 \%$ \\
\hline Financial Aid Obligations & 59 & $54.1 \%$ \\
\hline Multiculturalism & 23 & $21.1 \%$ \\
\hline Proximity to Extended Family/Relatives & 67 & $61.5 \%$ \\
\hline Proximity to Friends/Colleagues & 52 & $47.7 \%$ \\
\hline Regional and Recreational Activities Available & 72 & $67.9 \%$ \\
\hline Opportunity for Career Advancement & 54 & $49.5 \%$ \\
\hline Opportunity for Professional Experiences & 52 & $47.7 \%$ \\
\hline Desire to Return to Hometown & 18 & $16.5 \%$ \\
\hline Participation in Area-Specific Training Program & 14 & $12.8 \%$ \\
\hline Location of Previous Clinical Training/Residency & 15 & $13.8 \%$ \\
\hline
\end{tabular}

Table 3. Factors Important for Considering Rural Practice Sites ( $\mathrm{N}=109)$

\begin{tabular}{|l|l|l|}
\hline Factor & N & Percentage \\
\hline Income Potential & 83 & $76.1 \%$ \\
\hline Serving the Health Needs of the Community & 64 & $58.7 \%$ \\
\hline Influence of Spouse/Partner & 78 & $71.6 \%$ \\
\hline Quality of Education for Child(ren) & 87 & $79.8 \%$ \\
\hline Financial Aid Obligations & 54 & $49.5 \%$ \\
\hline Multiculturalism & 36 & $33.0 \%$ \\
\hline Proximity to Extended Family/Relatives & 71 & $65.1 \%$ \\
\hline Proximity to Friends/Colleagues & 68 & $62.4 \%$ \\
\hline Regional and Recreational Activities Available & 83 & $76.1 \%$ \\
\hline Opportunity for Career Advancement & 82 & $75.2 \%$ \\
\hline Opportunity for Professional Experiences & 77 & $70.6 \%$ \\
\hline Desire to Return to Hometown & 20 & $18.3 \%$ \\
\hline Participation in Area-Specific Training Program & 38 & $34.9 \%$ \\
\hline Location of Previous Clinical Training/Residency & 29 & $26.6 \%$ \\
\hline
\end{tabular}

Table 4. Factors Important for Considering Nonrural Practice Sites ( $=109)$

For the structural incentive questions, the majority of respondents agreed that adequate infrastructure at workplace (89.9\%), accessible geographic area \& transportation (89.0\%), and contact with medical technology were the most important incentives to potentially recruit them to practice in rural areas $(86.2 \%$, Table 5). Access to online information technology was ranked least amongst structural incentive options (68.8\%). Loan forgiveness options and guaranteed minimum incomes were the most appealing of the financial and personal incentives listed $(91.8 \%$, Table 6). Accelerated combined residencies were of least interest. The majority of the sample $(79.8 \%)$ would require at least $\$ 100,000$ of loans to be forgiven in order to consider loan forgiveness as a serious incentive option (Table 7). 


\begin{tabular}{|l|l|l|l|l|l|l|}
\hline Incentive Offered & $\begin{array}{l}\text { Strongly } \\
\text { Disagree }\end{array}$ & $\begin{array}{l}\text { Somewhat } \\
\text { Disagree }\end{array}$ & $\begin{array}{l}\text { Neither Agree } \\
\text { Nor Disagree }\end{array}$ & $\begin{array}{l}\text { Somewhat } \\
\text { Agree }\end{array}$ & $\begin{array}{l}\text { Strongly } \\
\text { Agree }\end{array}$ & $\begin{array}{l}\text { No } \\
\text { Response }\end{array}$ \\
\hline $\begin{array}{l}\text { Access to High- } \\
\text { Complexity Regional } \\
\text { Hospital }\end{array}$ & $2(1.8 \%)$ & $7(6.4 \%)$ & $13(11.9 \%)$ & $58(53.2 \%)$ & $28(25.7 \%)$ & $1(0.9 \%)$ \\
\hline $\begin{array}{l}\text { Free-Cost Drug } \\
\text { Treatments }\end{array}$ & $1(0.9 \%)$ & $4(3.7 \%)$ & $28(25.7 \%)$ & $51(46.8 \%)$ & $24(22.0 \%)$ & $1(0.9 \%)$ \\
\hline $\begin{array}{l}\text { High Income \& } \\
\text { Adequate } \\
\text { Accommodation } \\
\text { Facilities }\end{array}$ & $0(0.0 \%)$ & $3(2.8 \%)$ & $28(25.7 \%)$ & $29(26.6 \%)$ & $67(61.5 \%)$ & $1(0.9 \%)$ \\
\hline $\begin{array}{l}\text { Contact with } \\
\text { Medical Technology }\end{array}$ & $1(0.9 \%)$ & $3(2.8 \%)$ & $10(9.2 \%)$ & $43(39.4 \%)$ & $51(46.8 \%)$ & $1(0.9 \%)$ \\
\hline $\begin{array}{l}\text { Accessible } \\
\text { Geographic Area \& } \\
\text { Transportation }\end{array}$ & $2(1.8 \%)$ & $4(3.7 \%)$ & $5(4.6 \%)$ & $48(44.0 \%)$ & $49(45.0 \%)$ & $1(0.9 \%)$ \\
\hline $\begin{array}{l}\text { Access to Online } \\
\text { Information } \\
\text { Technology }\end{array}$ & $4(3.7 \%)$ & $8(7.3 \%)$ & $21(19.3 \%)$ & $35(32.1 \%)$ & $40(36.7 \%)$ & $1(0.9 \%)$ \\
\hline $\begin{array}{l}\text { Adequate } \\
\text { Infrastructure at } \\
\text { Workplace }\end{array}$ & $1(0.9 \%)$ & $2(1.8 \%)$ & $6(5.5 \%)$ & $37(33.9 \%)$ & $61(56.0 \%)$ & $1(0.9 \%)$ \\
\hline
\end{tabular}

Table 5. Level of Agreement to Interest in Structural Incentives ( $N=109)$

\begin{tabular}{|l|l|l|l|l|l|l|}
\hline Incentive Offered & $\begin{array}{l}\text { Strongly } \\
\text { Disagree }\end{array}$ & $\begin{array}{l}\text { Somewhat } \\
\text { Disagree }\end{array}$ & $\begin{array}{l}\text { Neither Agree } \\
\text { Nor Disagree }\end{array}$ & $\begin{array}{l}\text { Somewhat } \\
\text { Agree }\end{array}$ & $\begin{array}{l}\text { Strongly } \\
\text { Agree }\end{array}$ & $\begin{array}{l}\text { No } \\
\text { Response }\end{array}$ \\
\hline $\begin{array}{l}\text { Loan Forgiveness } \\
\text { Options }\end{array}$ & $1(0.9 \%)$ & $2(1.8 \%)$ & $5(4.6 \%)$ & $15(13.8 \%)$ & $85(78.0 \%)$ & $1(0.9 \%)$ \\
\hline $\begin{array}{l}\text { Guaranteed } \\
\text { Minimum Income }\end{array}$ & $1(0.9 \%)$ & $2(1.8 \%)$ & $10(9.2 \%)$ & $29(26.6 \%)$ & $66(60.6 \%)$ & $1(0.9 \%)$ \\
\hline $\begin{array}{l}\text { Accelerated } \\
\text { Combined } \\
\text { Residencies }\end{array}$ & $3(2.8 \%)$ & $9(8.3 \%)$ & $30(27.5 \%)$ & $32(29.4 \%)$ & $34(31.2 \%)$ & $1(0.9 \%)$ \\
\hline Tax Incentives & $0(0.0 \%)$ & $2(1.8 \%)$ & $13(11.9 \%)$ & $40(36.7 \%)$ & $52(47.7 \%)$ & $1(0.9 \%)$ \\
\hline $\begin{array}{l}\text { Reduced On-Call } \\
\text { Work }\end{array}$ & $1(0.9 \%)$ & $2(1.8 \%)$ & $18(16.5 \%)$ & $35(32.1 \%)$ & $52(47.7 \%)$ & $1(0.9 \%)$ \\
\hline
\end{tabular}

Table 6. Interest in Personal and Financial Incentives

\begin{tabular}{|l|l|l|}
\hline Amount Owed & $\mathbf{N}$ & Percentage \\
\hline$<\$ 50,000$ & 7 & $6.4 \%$ \\
\hline$\$ 50,000-\$ 100,000$ & 13 & $11.9 \%$ \\
\hline$\$ 100,000-\$ 150,000$ & 29 & $26.6 \%$ \\
\hline$\$ 150,000-\$ 200,000$ & 31 & $28.4 \%$ \\
\hline$>\$ 200,000$ & 27 & $24.8 \%$ \\
\hline
\end{tabular}

Table 7. Amount of Loan Forgiveness Required to Consider Loan Forgiveness as a Serious Incentive Option

Finally, using bivariate analysis, a chi-square test was conducted to examine association between previous residence in a rural area and personal motivation to practice in a rural area. A statistically significant relationship was established between these variables $(\mathrm{p}<0.001)$. Among those students who had previously lived, worked, or served in rural areas $(52.3 \%$ of the sample $), 86.2 \%$ had a personal motivation to practice medicine in a rural area (not shown in tables).

\section{DISCUSSION}

This was the first study to examine which factors would most heavily motivate current medical students to practice in medically underserved and rural areas of South Carolina. The study was innovative in its approach of sampling medical students studying in South Carolina, which can be informative for the medical practitioner rural recruitment process in the Southern region of the United States. ${ }^{16}$ The results from this study can be utilized to craft future state-supported incentive programs or to tailor current programs to more effectively recruit students to rural practice. 
Physicians are unlikely to consider rural practice without any incentives. ${ }^{5}$ This was true for this study, as the majority of students were not personally motivated to practice in a rural area or were unsure but would consider rural practice if sufficiently incentivized. Of respondents who had previously lived, worked, or served in rural areas, the overwhelming majority were personally motivated towards rural medical practice. These findings confirm previous research that rural exposure is related to high recruitment and retention rates of practitioners in rural areas ${ }^{1}$ and that rural backgrounds or connections are associated with willingness to work in a remote setting. ${ }^{13}$

Loan forgiveness options, guaranteed minimum incomes, and tax incentives were the most appealing of the personal incentives listed and were preferred more than non-financial incentives like reduced on-call work and accelerated residencies. The literature supports this finding, as guaranteed minimum income and loan forgiveness options have been found to be strong incentives. ${ }^{4}$ Loan forgiveness-based programs strongly support healthcare provider recruitment for rural practice, ${ }^{1}$ and loan forgiveness options were the most appealing personal incentive for the students in this study.

Respondents considered participation in area-specific training program and location of previous clinical training/residency to be the least important factors for rural practice. Medical school students usually do not feel comfortable practicing medicine in rural communities without some prior rural medicine training or exposure. ${ }^{6}$ The location of many healthcare training programs in urban areas may contribute to the paucity of providers in rural areas, as medical school students may learn in an urban environment and may not feel comfortable practicing medicine in rural communities. ${ }^{6}$ Many medical training programs maintain that they prepare healthcare professionals to practice anywhere; however, rural practitioners carry a heavier workload, provide a more diverse array of services, and carry a higher level of responsibility as a clinician practicing in relative isolation. ${ }^{7}$ Rural healthcare providers also have fewer diagnostic and treatment resources available to treat patients. ${ }^{1}$ For these reasons, rural practice requires specialized training that an urban medical education system may not be able to properly provide. Restructuring of the medical education system to eliminate urban bias, such as by including training in rural areas, could result in practitioners who are more willing to practice in rural areas. ${ }^{6}$

Open-ended questions about personal motivations for rural practice indicated students' previous rural experiences and personal connections to rural areas of South Carolina. Others expressed intrinsic philanthropic motivations: "[rural practice is] relevant and gratifying work, addressing medically underserved populations should be at the forefront of industry efforts." Some responses reflected financial motivations, such as better income opportunity or ability to pay off student loan debt.

The primary factors unique to considering rural practice were serving the health needs of the community and proximity to extended family/relatives. These results are also supported by previous studies where lifestyle considerations were found to be more important than financial incentives for practicing in a rural area. ${ }^{4}$ Community need was also ranked as an important consideration for rural practice and is supported by previous findings. ${ }^{1}$

There were limitations to this study. This research study provides insight for medical students in South Carolina but is not a representative sample of all medical students in the state. Our response rate $(<30 \%)$ is also a limitation. To accurately research what South Carolina medical students prefer in rural practice incentives, all medical schools should be included in the survey population. A state-supported program should also be derived from such research to best address the needs of all medical students in the state. The study also focuses exclusively on medical students and how to incentivize them to practice in rural areas. It does not examine the best incentives for other healthcare professionals such as nurses or physician's assistants, who are also targeted by rural incentive programs, and may not reflect their preferences for rural practice.

Finally, this study utilized an online survey to gather responses from the medical student population.

This method of data collection allowed for an increased number of respondents, but questions were left to respondents' interpretation, which might introduce some bias.

Additionally, respondents could have misinterpreted or misunderstood some questions while taking the survey. To build more upon the initial work of this research study, in-person interviews or focus groups should be conducted to follow up with respondents and confirm findings.

\section{CONCLUSIONS}

Through this study and the various studies conducted in different settings around the world, it is clear that there is no singular perfect solution to the problem of assessing how to recruit and retain physicians in rural communities. It is therefore imperative to assess the desires and preferences of populations targeted by incentive programs to determine which factors motivate them the most. Our study of 109 medical students attending the University of South Carolina School of Medicine adds unique information to this literature, indicating that an ideal rural practice incentive-based program for a medical school in South Carolina should be 
financial-based, using loan forgiveness, guaranteed minimum incomes, and tax incentives. For loan forgiveness options, as per respondents in this study, at least $\$ 100,000$ should be offered. In addition to financial-based incentives, clinic sites in areas with highest need should be well-supported to assure that practitioners have enough technology and resources to practice in relative isolation.

\section{ACKNOWLEDGEMENTS}

The authors thank the University of South Carolina School of Medicine for their cooperation and assistance in disseminating the survey to students, along with the medical students who participated in the study.

\section{REFERENCES}

1. Daniels, Z. M., VanLeit, B. J., Skipper, B. J., Sanders, M. L., and Rhyne, R. L. (2007) Factors in recruiting and retaining health professionals for rural practice, J Rural Health 23(1), 62-71. https:/ / doi.org/ 10.1111/j.1748-0361.2006.00069.x

2. Wilson N, Couper I, De Vries E, Reid S, Fish T, Marais B. (2009) A critical review of interventions to redress the inequitable distribution of healthcare professionals to rural and remote areas, Rural Remote Health 9(2), 1060.

www.rrh.org.au/journal/ article/1060 (accessed May 2018)

3. Geletko, K. W., Brooks, R. G., Hunt, A., and Beitsch, L. M. (2014) State scholarship and loan forgiveness programs in the United States: Forgotten driver of access to health care in underserved areas, Health 6(15), 1994-2003. http:/ / dx.doi.org/ 10.4236/ health.2014.615234

4. Hill, D., Martin, I., and Farry, P. (2002) What would attract general practice trainees into rural practice in New Zealand? $N Z$ Med J 115, (1161). http:/ / citeseerx.ist.psu.edu/viewdoc/download?doi=10.1.1.550.8230\&rep=rep1 \& type=pdf (accessed April 2018)

5. Jutzi, L., Vogt, K., Drever, E., and Nisker, J. (2009) Recruiting medical students to rural practice: Perspectives of medical students and rural recruiters, Can Fam Physician 55(1), 72-73, http:// wmw.cfp.ca/content/55/1/72.short (accessed April 2018)

6. Hutten-Czapski, P. (1998) Rural incentive programs: A failing report card, Can J Rural Med 3(4), $242-247$. https:/ / search.proquest.com/openview/4ce0cb325961151cd7ada461d5b68f33/1?pq-origsite=gscholare $\%$ cbl=45825 (accessed December 2017)

7. Strasser, R., and Neusy, A. J. (2010) Context counts: Training health workers in and for rural and remote areas, Bull $W$ orld Health Organ 88, 777-782. doi:10.2471/ blt.09.072462

8. Hartley, D. (2004) Rural health disparities, population health, and rural culture, Am J Public Health 94(10), 1675-1678. doi:10.2105/ajph.94.10.1675

9. Eberhardt, M. S., and Pamuk, E. R. (2004) The importance of place of residence: Examining health in rural and nonrural areas, Am J Public Health 94(10), 1682-1686. doi:10.2105/ ajph.94.10.1682

10. National Center for Health Statistics (2017) Health, United States, 2016: With chartbook on long-term trends in health, Report No. 2017-1232, National Center for Health Statistics, Hyattsville, MD.

11. Larson, E. H., Johnson, K. E., Norris, T. E., Lishner, D. M., Rosenblatt, R. A., and Hart, L. G. (2003). State of the health workforce in rural America: Profiles and comparisons, WWAMI Rural Health Research Center, University of Washington, Seattle, WA. https://depts.washington.edu/fammed/rhrc/publications/state-of-the-health-workforce-in-rural-america-profiles-and-comparisons/ (accessed February 2, 2018)

12. University of South Carolina School of Medicine (2017) FY 2018 USC School of Medicine Rural Health Presentation [PowerPoint slides], University of South Carolina. https:// www.scstatehouse.gov/Archives/CommitteeInfo/Ways\&MeansHealthcareBudgetSubcommittee/January312017/FY\%202018\%20 USC\%20School\%20of\%20Medicine\%20Rural\%20Health\%20Presentation.pdf

13. Honda, A., and Vio, F. (2015) Incentives for non-physician health professionals to work in the rural and remote areas of Mozambique-A discrete choice experiment for eliciting job preferences, Hum Resour Health 13(1), 23. bttps:// doi.org/10.1186/s12960-015-0015-5

14. Borracci, R., Arribalzaga, E. B., Couto, J. L., Dvorkin, M., Guerrero, R. A. A., Fernandez, C., Ferreira, L. N., and Cerezo, L. (2015) Factors affecting willingness to practice medicine in underserved areas: a survey of Argentine medical students, Rural Remote Health 15, 3485. www.rrh.org.au/journal/article/3485 (accessed October 2017)

15. Likert, R. (1932) A technique for the measurement of attitudes, Archives of Psychology 140, 1-55.

16. United States Census Bureau, Geography Atlas - Regions. https://www.census.gov/geo/reference/webatlas/regions.html (accessed May 2018) 


\section{ABOUT STUDENT AUTHOR}

Aalia Soherwardy is a recent graduate of the University of South Carolina as a part of the BARSC-MD seven-year dual degree program, with a concentration in Public Health. Aalia's research of rural healthcare recruitment and incentive programs was inspired by her exposure to rural medical practice and interest in increasing access to healthcare in rural areas. She is currently a medical student at the University of South Carolina School of Medicine.

\section{PRESS SUMMARY}

Individuals living in rural areas are more likely to have poorer health outcomes, but also have poor access to healthcare due to a shortage of providers in these locations. This is especially true for the rural areas of South Carolina and raises the question of how to attract providers to the rural Southern United States in a more efficient manner. The purpose of this study was to determine which incentives are most effective in motivating medical students to practice in rural areas of South Carolina. The results of this study can be utilized to create more effective rural incentive programs in the future or to tailor current programs to better recruit students to rural practice, especially in the Southern region of the United States. 


\section{APPENDIX A: Survey}

My name is __ [identifying information removed for review] and I am a USC Honors College student who will be a medical student at the University of South Carolina School of Medicine next year. For my honors senior thesis, I am collecting data about what factors work best to motivate medical students to practice in rural areas of South Carolina. The following questions will focus on what would incentivize practicing in rural areas most for you. When thinking of a rural area, please think of any area within South Carolina not considered to be inside the major metropolitan areas of Greenville, Columbia, and Charleston.

By proceeding to the next page, you agree to participate in the survey. The survey requires no identifying information from participants and is anonymous. You may withdraw participation at any time during the survey with no consequences. If you have already taken this survey before, PLEASE EXIT NOW.

1. What is your gender?

$\circ$ Female

- Male

2. What is your age?

- 17 or younger

- $18-20$

- 21-29

○ 30-39

○ 40-49

○ 50-59

- 60 or older

3. What is your ethnicity? (Please select all that apply.)

- American Indian or Alaskan Native

- Asian or Pacific Islander

- Black or African American

- Hispanic or Latino

- White/Caucasian

O Other

- Prefer not to answer

4. Which of the following best describes your current relationship status?

- Married

- Widowed

- Divorced

- Separated

- Single, but cohabiting with a significant other

- Single, never married

5. What level of medical education have you completed?

- First-year medical student

- Second-year medical student

- Third-year medical student

○ Fourth-year medical student

6. Roughly how much do you owe in student loans?

$\begin{array}{ll}\circ & <\$ 50,000 \\ \circ & \$ 50,000-\$ 100,000 \\ \circ & \$ 100,000-\$ 150,000 \\ \circ & \$ 150,000-\$ 200,000 \\ \circ & >\$ 200,000\end{array}$

7. Have you ever lived, worked, or served in a rural area?
- Yes

○ No

8. Are you interested in practicing primary care?
○ Yes
o No 
9. Select which of the following medical specialties you are interested in:
- Internal Medicine
- Obstetrics/Gynecology
- Pediatrics
- Family Medicine
- Other (please specify):

10. Do you have any personal motivations to practice medicine in a rural area? If yes, please specify:
- No
- Unsure
○ Yes (please specify):

If you answered YES to the previous question, go to question 12.

If you answered NO or UNSURE to the previous question, go to question 11.

11. If sufficiently incentivized, would you be interested in practicing in a rural area?
○ Yes
- No
- Unsure

12. Please indicate which of the following items are important when considering a practice location in a rural setting:

Income potential

- Serving the health needs of the community

- Influence of spouse/partner

- Quality of education for child(ren)

- Financial aid obligations

- Multiculturalism (presence of $>1$ culture in the community)

- Proximity to extended family/relatives

- Proximity to friends/colleagues

- Regional and recreational activities available

- Opportunity for career advancement

- Opportunity for professional experiences

- Desire to return to hometown

- Participation in area-specific training program

- Location of previous clinical training/residency

13. Please indicate which of the following items are important when considering a practice location in a nonrural setting:

- Income potential

- Serving the health needs of the community

- Influence of spouse/partner

- Quality of education for child(ren)

- Financial aid obligations

- Multiculturalism (presence of $>1$ culture in the community)

- Proximity to extended family/relatives

- Proximity to friends/colleagues

- Regional and recreational activities available

- Opportunity for career advancement

O Opportunity for professional experiences

- Desire to return to hometown

- Participation in area-specific training program

- Location of previous clinical training/residency

14. Below is a list of structural incentives that could be used to incentivize physicians to practice in rural areas. Please rate from 1 (strongly disagree) to 5 (strongly agree) if you would or would not be motivated by each item. 


Access to high-
complexity regional
hospital to refer patients
Free-cost drug
treatments for patients
High income and
adequate
accommodation facilities
Contact with medical
technology
Accessible geographic
area and transportation
Access to online
information technology
(for bibliographic search)
Adequate infrastructure
at workplace

15. Below is a list of personal incentives that could be used to incentivize physicians to practice in rural areas. Please rate from 1 (strongly disagree) to 5 (strongly agree) if you would or would not be motivated by each item.

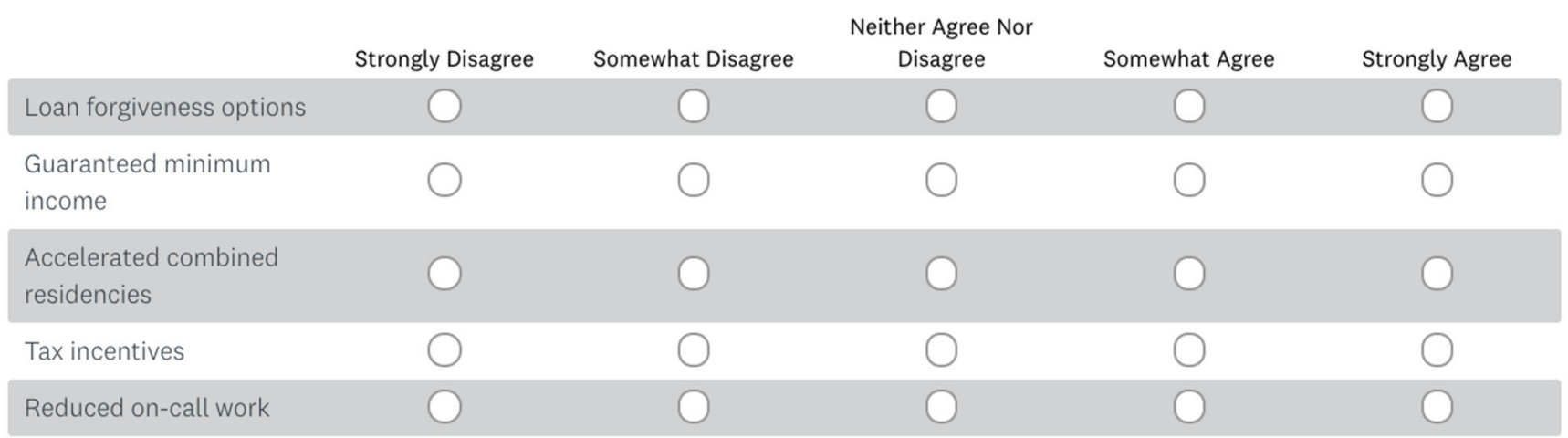

16. If loan forgiveness were an incentive option offered to you, how much of your loans would need to be forgiven?

$\begin{array}{ll}\circ & <\$ 50,000 \\ \circ & \$ 50,000-\$ 100,000 \\ \circ & \$ 100,000-\$ 150,000 \\ \circ & \$ 150,000-\$ 200,000 \\ \circ & >\$ 200,000\end{array}$




\title{
Travel Through Time: From 9/11 to COVID-19, Parallel Predictive Analysis of Travel Marketing
}

\author{
Hannah Gilliam* \\ Gainey School of Business, Spring Arbor University, Spring Arbor, MI \\ bttps://doi.org/10.33697/ajur.2020.029 \\ Student: hannabrg@gmail.com*
}

\begin{abstract}
The events of 9/11 drastically changed the state of the nation across many industry sectors, with the tourism industry among those most affected. Following that horrific day, the nation experienced heightened security measures and protocol, such that the travel industry and travelers would never look the same. People were fearful and anxious, and the tourism industry had to take quick, effective measures to evaluate the consumer response, set a marketing strategy, and promote within a changed national ethos and expectations. COVID-19 is a similar catastrophic, global, and long-term crisis that set our nation on a similarly drastic change in practice and protocol; fear and anxiety were higher than ever. COVID-19 and 9/11 are highly comparable in their market response. By comparing the two events and analyzing the consumer response and advertising messaging, specifically during the stay at home order, a theme and direction for messaging within the travel industry post-COVID-19 can be predicted based on the culture and spirit of The American Dream, confidence in safety, we are in this together, support local tourism, explore your city in a new way, and connect with those you missed.
\end{abstract}

\section{KEYWORDS}

COVID-19; 9/11; Post-pandemic; Advertising; Travel; Prediction; Messaging; Consumer Response; Marketing; Analysis

\section{INTRODUCTION}

Comparing 9/11 to COVID-19

External shocks, 9/11, and COVID-19 are comparable because they represent serious crises in consumer confidence over matters of safety and security across a broad spectrum of culture, economics, and travel. According to the official White House statement, "[on] March 11, 2020, the World Health Organization announced that the COVID-19 outbreak could be characterized as a pandemic, as the rates of infection continue to rise in many locations around the world and across the United States." Then, on March 13, President Donald Trump declared that the United States of America was in a National State of Emergency. After 9/11, President George W. Bush called a National State of Emergency due to terrorist attacks. These events led to an emergency declaration, which provides the legal justification for civil authority to enlist short term rules and requirements. However, this alone does not prove that these two events are comparable.

Both 9/11 and COVID-19 caused emotional distress and a crisis. The definition of a crisis is "“[a] serious incident affecting, for example, human safety, the environment or product or corporate reputation — and which has either received or been threatened by adverse publicity"'.1 9/11 was a direct threat to the safety of the United States. The attacks on 9/11 were received as a warning and caused a great turning point in the state of our nation, leading to fear and increased travel security. People were fearful for our country, for their lives, their families' lives, and fearful of flying. COVID-19 is a crisis too, first, the public health of our nation and the world. More than that, it is a crisis to the environment and to corporate reputation. Due to the economic crash, the second quarter was the worst of any quarter since recorded numbers with a Gross Domestic Product contraction at an annualized rate, ${ }^{2}$ there are many unknowns among the environmental and corporate worlds. People are once again fearful for their lives, their families' lives, and fearful of flying.

Bradley Johnson compiled a list of statements from an article written in 2001 after 9/11 regarding the government and public response to the crisis, ${ }^{3}$ and there are strong COVID-19 parallels:

\begin{tabular}{|l|l|}
\hline 9/11 Response & COVID-19 Parallel \\
\hline "There is one word for ad spending: uncertain." 3 & $\begin{array}{l}\text { Advertising, while still being pushed during the stay at home } \\
\text { order, had changed drastically. Companies were releasing } \\
\text { homemade ads, featuring employees filming themselves at } \\
\text { home. There is an unknown end to this pandemic, and firms }\end{array}$ \\
\hline
\end{tabular}




\begin{tabular}{|c|c|}
\hline & $\begin{array}{l}\text { do not know when to expect to increase their marketing and } \\
\text { advertising again. Microsoft Teams released a commercial } \\
\text { featuring their employees around the world, filmed from } \\
\text { home, speaking on how Microsoft Teams allowed them to } \\
\text { continue working. }\end{array}$ \\
\hline $\begin{array}{l}\text { "The terrorism attack also led to cancellations of sporting } \\
\text { events." } 3\end{array}$ & $\begin{array}{l}\text { During the initial wave of the COVID-19 pandemic, the } \\
\text { NBA season was canceled, and the Summer Olympics } 2020 \text { in } \\
\text { Tokyo was postponed to } 2021 \text {. }\end{array}$ \\
\hline $\begin{array}{l}\text { "The commissioner of baseball canceled all Major League } \\
\text { games. The Emmy Awards were postponed. Broadway shows } \\
\text { were shuttered". 3 }\end{array}$ & $\begin{array}{l}\text { Many of the MLB games have been canceled, including spring } \\
\text { games and training. }{ }^{5} \text { NFL drafts were virtual. }{ }^{6} \text { Many award } \\
\text { shows were rescheduled, including the Pulitzer Prize, the } \\
\text { Emmys, and the Tonys. Broadway shows were all shut down. }{ }^{7}\end{array}$ \\
\hline $\begin{array}{l}\text { "Movie studios will make some marketing cutbacks in the } \\
\text { short term as they postpone releasing some films with } \\
\text { terrorist or violent content." } 3\end{array}$ & $\begin{array}{l}\text { We have yet to see fully how movie studios will change } \\
\text { marketing due to the material and subject matter of a movie; } \\
\text { however, many studios proceeded forward with online and } \\
\text { streaming services for a virtual premiere of a film. For } \\
\text { example, in early September, Disney Plus did a virtual } \\
\text { premiere of the new live-action Mulan. }\end{array}$ \\
\hline $\begin{array}{l}\text { "The travel category faces dim near-term prospects given } \\
\text { terrorism and economic woes." } 3\end{array}$ & $\begin{array}{l}\text { Travel was shut down except for specific cases, and borders } \\
\text { across the world were closed. }\end{array}$ \\
\hline $\begin{array}{l}\text { "Dozens of conventions and trade shows were canceled this } \\
\text { month due to the terrorist attacks in New York and } \\
\text { Washington." } 3\end{array}$ & $\begin{array}{l}\text { The Las Vegas Trade Show was shut down, and many state } \\
\text { fairs were canceled. }{ }^{10} \text { Oktoberfest, in Germany, was canceled } \\
\text { for } 2020 .{ }^{11} \text { The historic St. Patrick's Day parade in Dublin, } \\
\text { Ireland was canceled in } 2020 .^{12}\end{array}$ \\
\hline "Video conferencing [is rising] as some are not ready to fly." 3 & $\begin{array}{l}\text { Zoom, Webex, and other video conferencing media are in use } \\
\text { every day across the globe. Apps for video conferencing hit a } \\
\text { high download rate of } 62 \text { million one week in March, } 2020 .^{13}\end{array}$ \\
\hline
\end{tabular}

Table 1. 9/11 and COVID-19 parallel cultural comparisons.

\section{METHODS AND PROCEDURES}

Regarding the current state of the economy and the world, this study examines how the COVID-19 pandemic will affect the travel industry and how the industry will promote, advertise, and ensure confidence in their consumers. To confidently predict what will happen, it is important to look to another time when the travel industry faced consumers fearing for their safety. Through peer reviews and case studies, research was drawn together on the 9/11 terrorist attacks and their advertising effects.

\section{RESULTS}

Company Approaches

Crisis Strategies: A Comparison

The attacks on $9 / 11$ were a crisis. When dealing with a crisis, there are a few ways to identify the crisis and then decide how to proceed. Evans \& Elphick from International Journal of Tourism Research, ${ }^{1}$ article "Models of crisis management: an evaluation of their value for strategic planning in the international travel industry" discuss these differences.

\begin{tabular}{|l|l|l|l|}
\hline Seymour and Moore & Response & Booth (1993) & Response \\
\hline $\begin{array}{l}\text { The 'Cobra' type of crisis is } \\
\text { sudden, for example, a } \\
\text { disaster, which may come as a } \\
\text { shock (e.g., September 11) }\end{array}$ & $\begin{array}{l}\text { Defensive response with } \\
\text { reliance on the known and } \\
\text { trusted }\end{array}$ & $\begin{array}{l}\text { Sudden threat or loss to the } \\
\text { whole organization }\end{array}$ & $\begin{array}{l}\text { Defensive response with } \\
\text { reliance on the known and } \\
\text { trusted }\end{array}$ \\
\hline $\begin{array}{l}\text { The 'Python' type of crisis } \\
\text { gradually creeps upon a } \\
\text { company, for example, } \\
\text { caused by poor management } \\
\text { or high costs. }\end{array}$ & $\begin{array}{l}\text { Bureaucratic response when } \\
\text { the crisis is not recognized- } \\
\text { negotiated response when } \\
\text { crisis recognized }\end{array}$ & $\begin{array}{l}\text { Periodic threat or loss to part } \\
\text { or whole of the organization } \\
\text { Gradual threat to part of the } \\
\text { organization }\end{array}$ & $\begin{array}{l}\text { Negotiated response and } \\
\text { recognition of the problem } \\
\text { crisis is not recognized }\end{array}$ \\
\hline
\end{tabular}


According to Table 2, 9/11 is a Cobra crisis because the terrorist attacks were sudden and surprising. People, businesses, and the government were unprepared. This is the biggest difference between 9/11 and COVID-19. COVID-19 is a Python crisis. The first known case of COVID-19 can be traced back to November 2019. It was declared a pandemic in the United States on March 11, 2020. This shows the slow, gradual onset of the crisis. Because of this, there are monumental, long-term effects that perhaps the nation did not see as a result of $9 / 11$.

\begin{tabular}{|c|c|c|}
\hline Model & Approach & Limitations \\
\hline Caplan's (1970) crisis model & $\begin{array}{l}\text { Psychological perspective, whereby the } \\
\text { focus is on how the individual copes } \\
\text { with a crisis }\end{array}$ & $\begin{array}{l}\text { The model lacks precision and is } \\
\text { descriptive. The most important } \\
\text { criticism is that it is homeostatic }\end{array}$ \\
\hline $\begin{array}{l}\text { Slatter's (1984) crisis susceptibility } \\
\text { model }\end{array}$ & Economic approach to crises & $\begin{array}{l}\text { It suggests only the factors that are } \\
\text { susceptible to a crisis in an organization. } \\
\text { It is not a process, merely a model } \\
\text { stating factors that may cause a crisis }\end{array}$ \\
\hline Arnold's (1980) model of crisis & $\begin{array}{l}\text { Sociological perspective and looks at } \\
\text { how communities react to crisis }\end{array}$ & $\begin{array}{l}\text { Only focuses on the sociological view } \\
\text { and centers on the individual in relation } \\
\text { to a group. The way an individual views } \\
\text { the crisis may be different to the } \\
\text { organization }\end{array}$ \\
\hline $\begin{array}{l}\text { Process model of crisis development } \\
\text { (Booth, 1993) }\end{array}$ & $\begin{array}{l}\text { Aims to identify features that appear to } \\
\text { be common in many crises }\end{array}$ & $\begin{array}{l}\text { Too general and simple - all crises are } \\
\text { unique in terms of the particular causes } \\
\text { and effects involved }\end{array}$ \\
\hline $\begin{array}{l}\text { The crisis life cycle (Seymour and } \\
\text { Moore, 2000) }\end{array}$ & $\begin{array}{l}\text { Looks at the obstacles to decision } \\
\text { making during a crisis }\end{array}$ & $\begin{array}{l}\text { Too descriptive and general - although } \\
\text { can be made to fit any organization }\end{array}$ \\
\hline Clarke and Varma (2004) & $\begin{array}{l}\text { Presents a model of risk management as } \\
\text { a strategic process }\end{array}$ & Difficult to put into operation \\
\hline $\begin{array}{l}\text { Model of crisis management (Smith, } \\
\text { 1990; Smith and Sipika, 1993) }\end{array}$ & A process from start to finish of a crisis & May be too general and descriptive \\
\hline
\end{tabular}

Table 3. Crisis Management Models, Approach, and Limitations. ${ }^{1}$

Evans \& Elphick then go on to explain crisis models that have been executed, as well as their approaches and limitations (see Table 3). Arguably, 9/11 followed Caplan's (1970) model: "Psychological perspective, whereby the focus is on how the individual copes with a crisis." Many aspects of the nation were affected after 9/11; arguably, the biggest effect was air travel and security changes. With TSA policies in place, the government was focused on how to calm people's nerves, fears, and anxieties about the safety of their country and their aircraft.

At the time of this writing, the COVID-19 pandemic was still in the first wave regarding the travel industry. The same model will likely be applied. People were fearful of traveling out of their houses, let alone across the world. When the time comes that widespread travel is allowed, the travel industry will need to have a crisis model prepared. While the government will most likely place restrictions on travel and new policies, it is up to the travel industries and each firm to instill confidence and safety to the individual. Evans \& Elphick outline the limitations of Caplan's crisis model, stating: "the model lacks precision and is descriptive. The most important criticism is that it is homeostatic." Once the world is fully running again, the travel industry must be quick on its feet to adjust to the consumer emotion. They cannot merely put policies in place, such as with $9 / 11$; they must constantly be responding to the evolving consumer response.

After 9/11, internal communication was a top priority. Argenti (2019) wrote an article for the Harvard Business Review in which he worked with managers to understand the crisis response, specifically to $9 / 11$. He states initially: "What I discovered is that, in a 
time of extreme crisis, internal communications take precedence. Before any other constructive action can take place-whether it is serving customers or reassuring investors — the morale of employees must be rebuilt". ${ }^{14}$ This firmly held true regarding the COVID-19 crisis. Internal communication was, and is, key. Hospitals sent out new policies and updates every few days to their employees. Before the stay-at-home order, employees were being informed of every change that was taking place.

Argenti then continues to lay out a five-step process for a company facing post-crisis:

Get on the scene. The most important thing after a crisis is to jump onto the scene immediately. This can mean press conferences or statements. It is important to stay visible to the customer. Concerning $9 / 11$, there were many press conferences and speeches made all over the nation in the days following. "'When people heard us on the speakers, they listened. Your voice must sound calm, in control and, most important, earnest,' says Lewis, thinking back...' most of all, we wanted people to know we were all in the same boat"'.14

This concept remains true for COVID-19. Many companies and firms released statements on their positioning or availability of service due to the pandemic. Everyone heard from their bank, their doctor, the drug store, even retail stores. Companies that were still offering services kept extremely high visibility. Printing signs that read "OPEN FOR DELIVERY," among other postings.

Choose your channels carefully. "Whether natural or man-made, disasters often disrupt normal flows of communication."14 For 9/11, one of the internet companies crashed, and cell towers were down, which had a huge effect on lines of communication within New York. "[M] any of them realized that they needed to start thinking of the media as allies-in part because their failed communications systems left them no other choice". ${ }^{14}$

For COVID-19, digital and media communications were the only flows of communication. There were live streams daily from government officials and doctors. Churches were live streaming services. People were stuck in their homes, and they were bored. They were turning to digital communication for information, statements, and entertainment. Many companies decided to release and stream fun challenges, ads, or memes to keep the consumer's attention.

Stay focused on the business. During 9/11, it proved effective to have people focus on work. People felt lost and unsure, and so having "an outlet for their desire to help, gets them back into a normal routine, fosters their pride in the company and what they do, and builds strong bonds between themselves and their customers, many of whom desperately need the company to keep their products and services flowing". ${ }^{14}$ By having somewhere to place their energy, people felt not only productive, but also felt helpful and beneficial to the greater cause.

In the first wave of COVID-19, many people were out of work or working from home. This has had a large effect on the economy, and it also has a huge effect on morale. Companies began to find creative ways for people to feel productive and beneficial. There were challenges to complete that raised awareness. Companies were selling specific new product lines with proceeds going to COVID-19 relief. Even celebrities were auctioning off events to raise donations. This brought aid to those in need, but it helped them stay focused on business and the greater good on the consumer side.

Have a plan in place. Argenti urges strongly for the use of web-based communication and for contingency planning. Even with intranet servers down, there needed to be strong communication. "Although operations during a crisis should be decentralized, decision making should not be." Decision-making must continue to be strong and centralized amongst the chaos.

COVID-19 has proved this idea many times over. All nonessential firms decentralized operations and offices, but the company's decision-making and forward motion needed to continue. This is why so many people could continue to work from home.

Improvise but from a strong foundation. "CEO John Murphy says, 'If you have a strong culture, you can maintain focus. On 9/11, we had a structure, a belief system, and a hierarchy all in place. That helped us to get through the crisis, and we have not skipped a beat since"'. ${ }^{14}$ Because of the strong foundation, after $9 / 11$, firms were able to move forward and show the consumer the future through the eyes of their belief system.

During COVID-19, firms acted very quickly. For example, a medical device company, Stryker, was able to push out an emergency bed in 10 days from original conception to when the bed was in the hospitals. Many companies, specifically 
insurance, travel, and cell, had advertising out that amplified their belief in bringing people closer together during this time. These industries believe in community power; during this time of social distancing, they released ads that created emotions of togetherness and familiarity. They told the consumer that "we want to bring you together virtually and keep you safe right now."

\section{Effect on the Travel Industry}

Being as the major vehicle in the terrorist attacks of 2001 were airplanes, the travel industry took a huge economic hit afterward. Right after the attacks, all commercial flights were grounded for three days. This "resulted in a 31.6 percent reduction in travel volume in September of 2001 compared to that same month in 2000 and generated massive industry losses". 15 People were afraid to fly but were still willing to travel. Due to COVID-19, most commercial flights were canceled during the stay-at-home order, and we do not yet know the complete economic effect this will have. The biggest difference during COVID-19 is that people are afraid to travel, not just to fly. People are afraid to go anywhere, and this will cause major impacts on the lodge industry, tourism industry, restaurant industry, tour operators, and the airline industry. These all combine to create a broader impact on the travel industry.

\section{DISCUSSION}

\section{Consumer Emotional Response}

The initial consumer response to 9/11 was intense. People were scared and shocked. People were confused by what had happened; it was sudden and unexpected. People were anxious and fearful of what the future, as well as the present, held. Many questions were unanswered. This, of course, led to people being fearful of flying. Even a year after the attacks, the consumer response was the same. "U.S. consumers are now more wary of foreign travel, with 39\% saying they are less likely to travel abroad, according to Euro RSCG Worldwide's Mind and Mood Monitor. They regard 'home as a haven"' ${ }^{16}$ The phrase "home as a haven" is very powerful. Consumers turned to the emotional pull of "we are American." There was great pride in how the country bound together and linked arms to be one nation, one home. This is not something that happens very often in the United States. There must be a strong enough emotional response. This nationalism and patriotism ran through homes, stores, and the government. As this wave was felt, " $50 \%$ of people aged over 50 say they are now more likely to 'buy American"'. ${ }^{16}$

The initial consumer response to COVID-19 was a lot slower because the crisis was a Python crisis. The emotional response was annoyance that changed into fear and anxiety, fear for the world's unknown state and the nation for both the present and the future. The first response to airlines and travel was to buy as many good deals as possible; however, when the reality of the situation settled in, people were ready to travel but scared of the actual act and the consequences. "Home is haven" quite literally during stay-at-home state orders. While people may not have been emotionally content with this, they were fighting for everyone who could not stay home. There is a nationwide awareness that every individual's role is to play the part for the greater good and create a national haven and safe space. There is a rise in patriotism, a patriotism that is different from before; it is not Americans against terrorists but Americans against an untouchable disease. This is not to say it is better or worse, just that it feels different because every American has directly felt the effects of this pandemic. "America has long equated patriotism with the armed forces. However, you cannot shoot a virus. Those on the frontlines against coronavirus are not conscripts, mercenaries, or enlisted men; they are our doctors, nurses, pharmacists, teachers, caregivers, store clerks, utility workers, small-business owners, and employees. When all is said and done, perhaps we will recognize their sacrifice as true patriotism, saluting our doctors and nurses, genuflecting". ${ }^{17}$

"Shop Local" has been a very important phrase in the media right now. This parallel to "buy American" is not lost. People want to support their neighbors, near and far. The fact that this is happening amid the pandemic only predicts that it will continue forward as life adjusts back to normal. When people feel safe enough to travel, they will travel for one reason to support communities and local businesses. People will want to experience culture and art through the "underdog." "Localism is likely to be a much stronger factor, particularly in the early stages of recovery. Not only will this assist with social reconnection, it is [sic] a more sustainable model that supports climate change adaption [sic]". ${ }^{18}$

\section{Market Response}

Budget response. One of the biggest responses to 9/11 within the market was asking, "why do we spend money on advertising?" With the change in dynamics and media, there were big discussions about where and what advertising money was being spent:

Budget Cuts. The increased reliance on public relations may be attributed to the need to reduce budgets for advertising and marketing, which traditionally use strategies and tactics that are more expensive than public relations, rather than 
public relations' expertise regarding persuasive appeals and message structure. One major reason for the budget shift appears to be less bed tax revenue due to low hotel occupancy. ${ }^{19}$

This question of advertising goes into evaluating where the most effective use of money will be to be able to draw people back into the market after a crisis. "We shifted our budget greatly to funnel more funds into advertising and public relations that would convince a "scared" public that travel is safe and appropriate". ${ }^{19}$ Advertising budgets have already shifted because of COVID-19 while firms decide how consumers will respond.

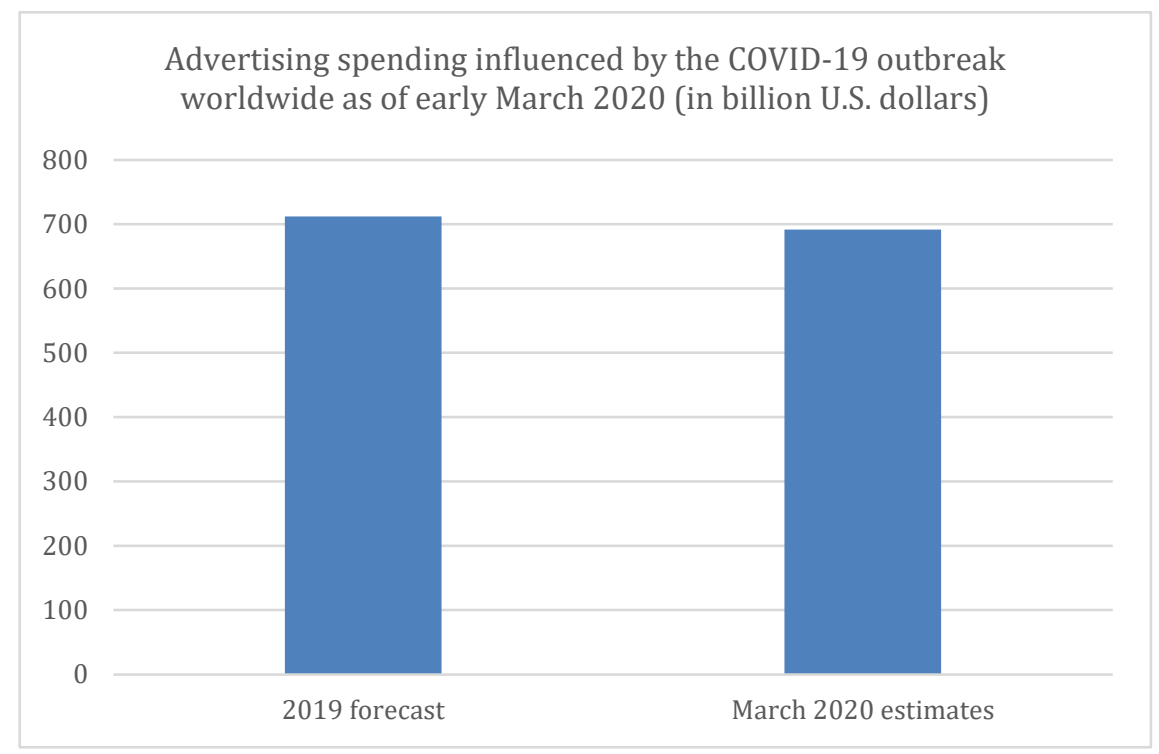

Figure 1. Advertising Spending Influenced by COVID-19 as of early March $2020^{20}$

Pre-epidemic March 2020

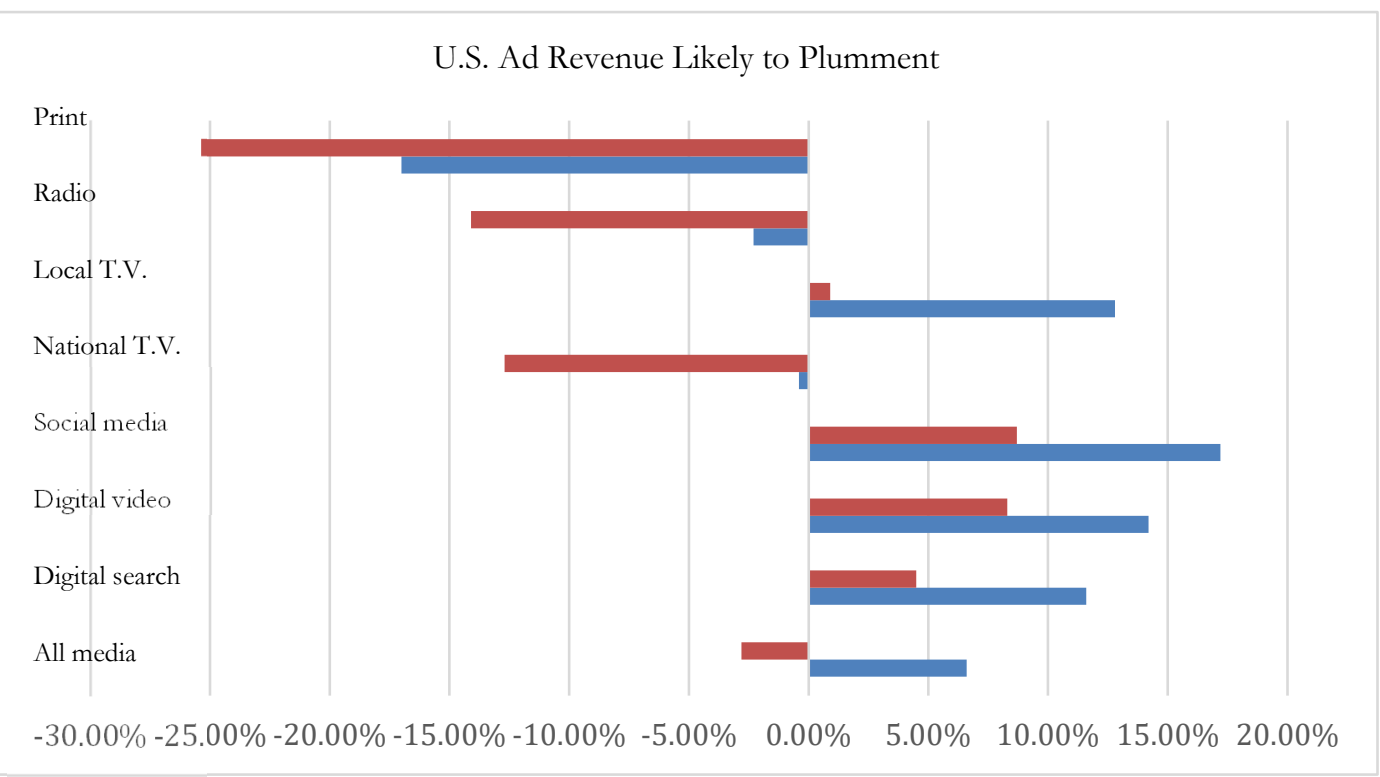

Figure 2. U.S. Ad Revenue Likely to Plummet Pre-epidemic vs. March 202021

Geographical travel response. Another big market response to 9/11 was that everyone was driving and traveling closer to home. "According to a Travel Industry Association of America (TIA) report on changing travel patterns, three-fourths of its members are seeing an increase in closer-to-home travel and last-minute travel among their customers". ${ }^{19}$ Everyone transitioned to close to home adventures. When it came to travel, people were concerned with the destination and the emotions related to it, and so they would sacrifice, no matter how far, in order to be in tune with those emotions. "[D]estination image should incorporate both cognitive (beliefs and knowledge about a travel destination's attributes) and affective facets (emotion or feelings attached to the 
destination)...destination image is a significant mediator between perceived travel risks and visit intention". 18 The important objective was to draw close together as a nation. The only way people were traveling was if the emotional pull outweighed the travel risk.

We can predict that the same situation will happen with travel after COVID-19. Consumers that do travel will need a high emotional pull to that specific destination. Anyone who travels by plane, to be in tight quarters with strangers for hours, will need to have an even greater emotional drive to overthrow the fear.

Travel Advertising and Messaging After Crisis

Advertising Strategies After $9 / 11$

Promotional push instead of pull strategy. Because of emotions of fear, anxiety, and anger, it was very effective to use push marketing and advertising to push people into travel. "[W] hile pull factors refer to those that lead an individual to select one destination over another once the decision to travel has been made. Push factors are viewed as relating to the needs and wants of the traveler... Pull factors, on the other hand, have been characterized in terms of the features, attractions, or attributes of the destination itself... (p. 385)". ${ }^{19}$ These push factors must be strong enough to once again overcome the travel fears. The advertiser needed to push the traveler past the psychological aftermath. Companies quickly ran campaigns, not only to get their message out immediately but also because "there [was] an unspoken awareness that another September 11—God forbid—could cause more lasting damage. There's a slight sense of urgency, of making hay while the sun shines". ${ }^{22}$

For the tourist traveler. For the travel industry, they also needed to run push campaigns. The travel and tourism industry normally focuses on pull factors: drawing the consumer in with events and mass media. This is not effective during moments of high emotion, tension, or crisis. For the travel industry as a majority, most of them could successfully advertise to consumers if they could avoid airlines. "As a result, $87 \%$ of its member organizations have changed their marketing or promotion programs, with $77 \%$ focusing their efforts on closer, drive-in markets during the last 12 months (Keefe, 2002a)". ${ }^{19}$ However, the airlines had to push even harder to try and compensate for the emotional toll.

The travel industry needed to focus on the push or emotional effect of entertainment in each destination:

'People want to get back to what is real. I plan to not focus on entertainment as much as the way people feel while they are in Dickson County.' Human interest and "feel good" stories also are being used by a major tourist attraction in the Southeast, and an attraction in the West reported that it is 'using more visual imagery-our animals, bright colors, happy people-to better relay [a] sense of fun and wonder. ${ }^{\prime 19}$

For tourism in general, there needed to be a sense of normalcy and a heightened normalcy-fun. People needed a break and release from the emotional strains of the attacks:

The key to activating local visitor economies will be to increase demand, but (even assuming this would work with the entire world competing for scarce tourism spend) intensive marketing to attract large numbers of visitors may overwhelm the limited resources and capacity of the remaining businesses. Instead, the initial focus should be on visitor segments that can function happily within the constrained environment, spending money in the grassroots businesses (cafes, shops, wineries) that underpin the tourism sector. Similarly, after the total closure of music venues (with many not expected to re-open if travel/social restrictions extend for several months), new venues for live music and entertainment will be needed, presenting opportunities for co-creation of cultural experiences in wineries, cafes, galleries and local pubs. ${ }^{18}$

The travel industry also needed to focus on a destination's localism, such themes discussed earlier as in "the Dream American" and "home as in haven." The industry gave space for the consumer to see and support the local tourism, businesses, and economies of each destination. Furthermore, the advertising needed to create a sense of community.

For the corporate traveler. Messaging and strategy for the corporate traveler are slightly different; however, focusing on the corporate traveler may be what keeps a company from going under. In many cases, this travel is vital, and so the competition for that consumer is high. The corporate traveler will be pushed with promotions and deals, so a company must rise on time with a promise of improved safety and service. The importance is to figure out exactly what those improvements look like. "[T]he ability of the travel service provider to contribute to effective business outcomes and the safety of the traveler". ${ }^{21}$ 
Messaging After 9/11

Through looking at the consumer's emotional response to $9 / 11$, some of the main messaging has already become clear: home as in haven, buy American, and patriotism.

American Dream. The first messaging is the American Dream and patriotism. Even restaurants were using this messaging to push consumers to go out to eat. '[T] he 'I Love New York' and other marketing campaigns'... 'We have several hundred events scheduled this year, everything from festivals to performing arts, stage plays, and the new film center in Pleasantville. We hope these will attract even more visitors this year"', 23

G.M. advertising introduced us to this concept, as they were one of the first to carry this messaging. They drew on the American Dream and ended up breaking auto sale records in October 2001.19 "G.M. acted forcefully with a marketing campaign and financial incentives at the time when many marketers were tentative and uncertain". 3

Figure 3 GM Advertisement ${ }^{24}$

https://www.marketplace.org/2020/04/03/advertisers-aretrying-adapt-covid19/

Confidence in safety. The next messaging was to instill confidence and inform the consumer about the changes and safety precautions in place-precautions that would push past the emotional trauma. Tampa International Airport was able to implement this with the American Dream. They ran a campaign with the slogan "'Keep Flying America' that was implemented to educate the public about changes in airline travel since $9 / 11$ and to help relieve the fear of flying". ${ }^{25}$

American Airlines, one of the airlines used in the attacks, ran two campaigns. ${ }^{25}$ The first campaign was a commercial that, over a minute, read "We are an airline. But it has become clear we are more. We are a way of life. The freedom to come and go, anywhere, anytime, with confidence and peace of mind. We are an airline that is proud to bear the name: American." They pushed forward with pride in their company and their nation. They also conveyed the importance of feeling confident and peaceful about your travel.

We are in this together. The final messaging is that we are all in this together. American Airlines' second campaign consisted of three commercials with the slogan "The Great American Get-Together. Be a part of it," with discounts for in-country flights. Each video boasted its own theme, respectively: family, friends, and getaway. Each video talks about people that are "somewhere in America." The family video named different individuals and activities like a camera-shy mom and muddy backyards that will become football fields. The friends' video shows the breadth of a lifelong friendship from young children to old friends: "You knew you'd be back, you'd all be back together. Somewhere in America." The getaway video talks about how somewhere there is a lack of errands, homework, but time, memories, and journeys are bound to be brought together. "Somewhere in America...the best way to get together is simply to get away."

United Airlines ran a campaign: We are United. Different employees talked about why they do what they do and how they are here for us. "We took a blow... but we are going to get up, and stand up, and press on."

Market Response and Advertising Already in Place for COVID-19

Because COVID-19 is still currently a crisis worldwide as of November 2020, there must already be advertising in place.

Reviewing this starts with the market response statistically, and then the messaging already in use.

Statistics

Focus and uses of media have shifted. Consumers were bored and at home with nothing to do. See Figure 4 to watch the rise of on-demand media as people searched for mindless ways to occupy their time. Figure $\mathbf{5}$ showcases how specifically the older population was filling their time with news channels. People were scared and over researching the virus and were hyper-aware of the news.

\section{Messaging}

Stay At Home. Main messaging circulates themes regarding the stay-at-home order. Companies were trying to stay visible in front of consumers. Big companies were taking the responsibility of pushing people into their homes. Through this, they were staying at the front of the mind for the consumers. WhatsApp had a commercial that even celebrates the togetherness that is possible during social distancing: "In the Distance, Stay Close." Coca Cola (Figure 6) encouraged people that by staying apart, they are uniting the human race. Nike had a similar play off of this theme by showing that staying inside unites humanity as one team or audience (Figure 7). Toyota had parallel messaging as the following Honda ad (Figure 8). Toyota had a South Africa commercial running that shares how much they love to get people moving but being home has never felt so right: "We Look Forward to Everyone Being Back on the Road." McDonald's (Figure 9) does not have any direct underlying themes but encourages 
separation by altering their logo. Finally, Spotify (Figure 10) encouraged social distancing by offering music to relieve the stress and loneliness of COVID-19.

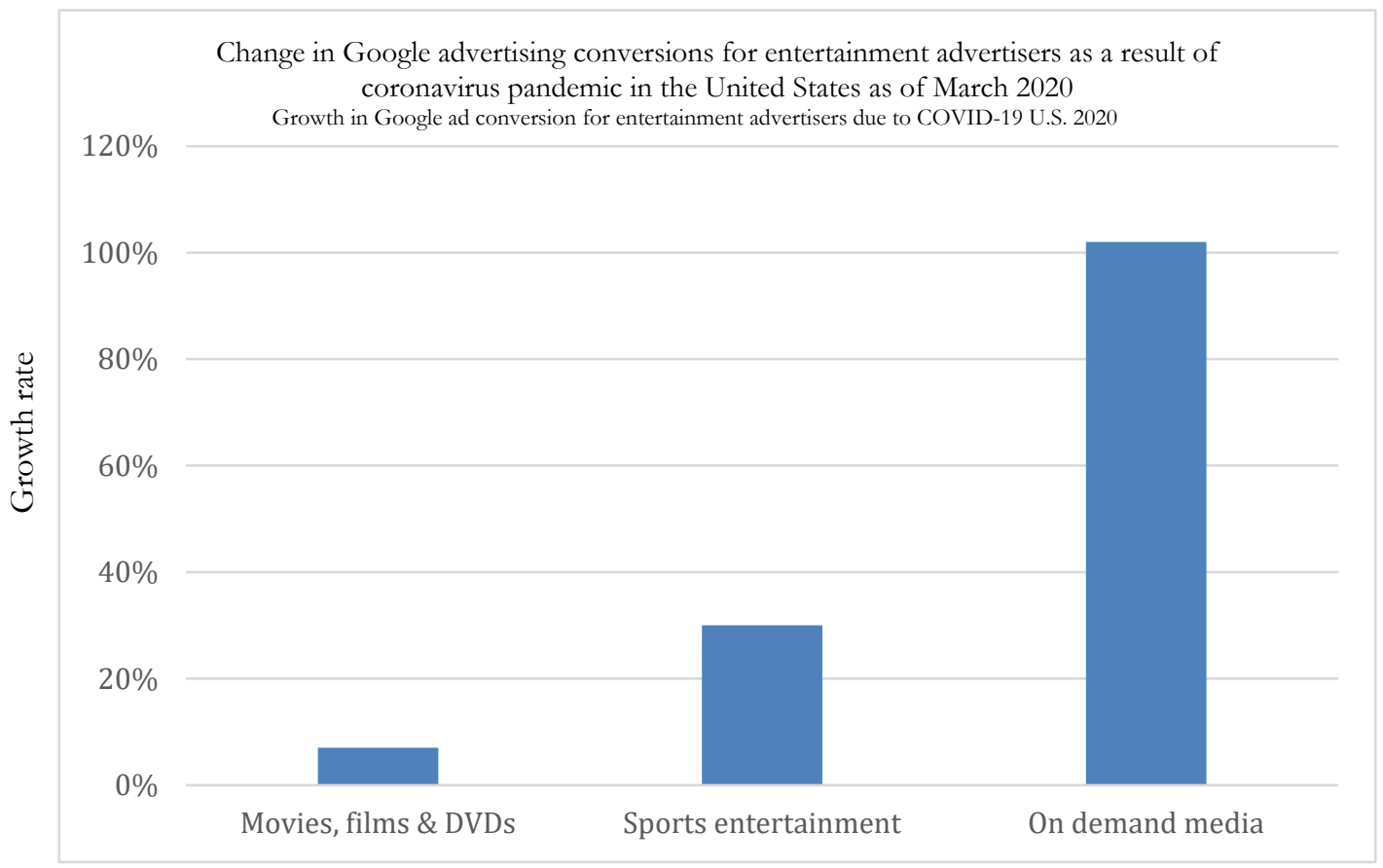

Figure 4. Change in Google Advertising as a Result of COVID-1926

Aged 25-54

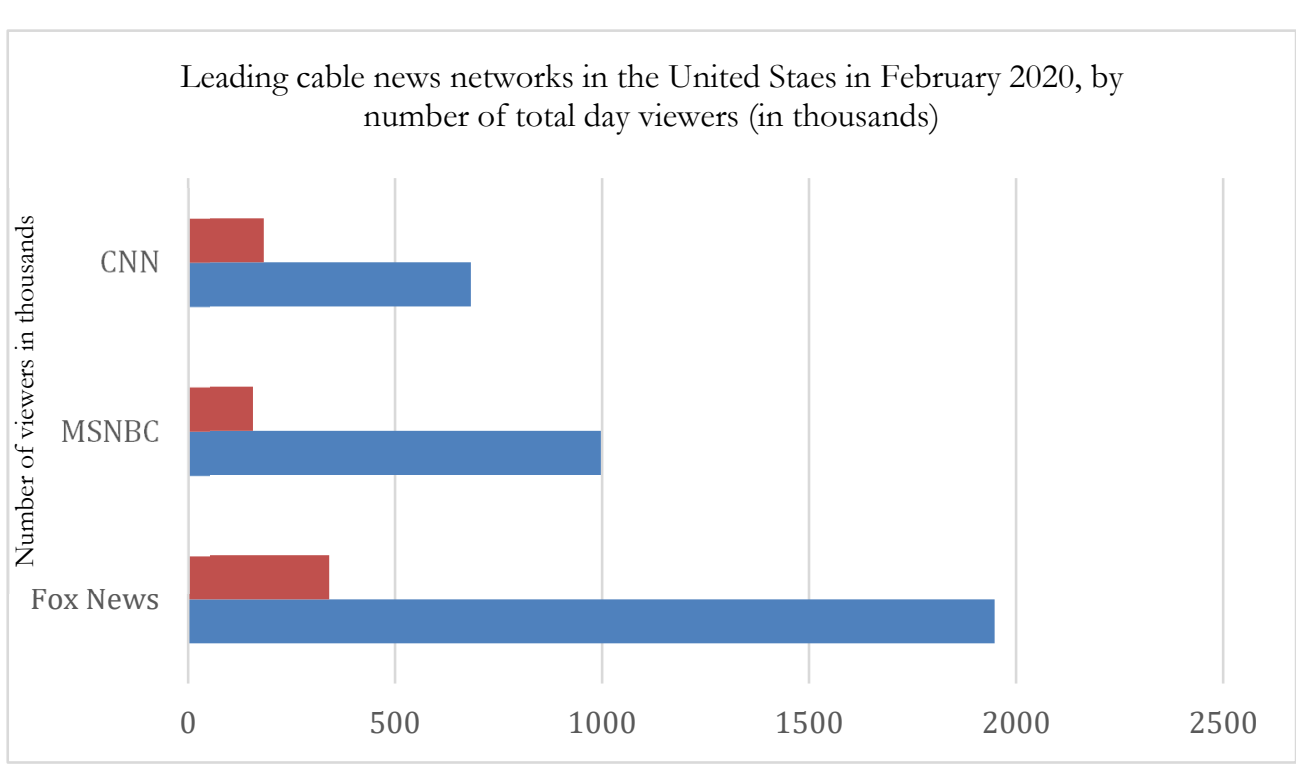

Figure 5. Leading Cable News Networks as of February 202027

Figure 6. Coke $\mathrm{Ad}^{28}$

Figure 7. Nike $\mathrm{Ad}^{29}$ https://www.campaignlive.com/article/coke-goes-big-covidsocial-distancing-times-square/1677909

https://www.gartner.com/en/marketing/insights/dailyinsights/how-active-are-activewear-brands-in-the-fightagainst-covid-19 


\begin{tabular}{|l|l|}
\hline Figure 8. Honda Ad ${ }^{30}$ & $\begin{array}{l}\text { https://auto.hindustantimes.com/auto/news/stay-home- } \\
\text { honda-promotes-covid-19-awareness-with-this-homemade- } \\
\text { civic-commercial-41587186158063.html }\end{array}$ \\
\hline Figure 9. McDonald's Ad ${ }^{31}$ & $\begin{array}{l}\text { https://www.today.com/food/mcdonald-s-changes-golden- } \\
\text { arches-logo-amid-coronavirus-outbreak-t176653 }\end{array}$ \\
\hline Figure 10. Spotify Ad ${ }^{32}$ & $\begin{array}{l}\text { https://techcrunch.com/2020/03/25/spotify-adds- } \\
\text { fundraising-features-and-a-covid-19-news-hub-to-address-the- } \\
\text { health-crisis/?guccounter=1 }\end{array}$ \\
\hline
\end{tabular}

We are in this together. The biggest messaging theme is we are in this together. Once again, we see the parallelism of bringing people together and patriotism rising to draw together our strength. Companies are pushing that they are here with the consumer. They are walking alongside them, and they will be there through every step. Through this, they show they will be there for the steps afterward. Ford led the way on this messaging with a commercial that named everyone affected, followed by a link for support from Ford. Then the screen read "Built for America." This is patriotism and huge togetherness. Jack Daniels had a commercial running that reads "Dear Humanity, Here's to social distancing, socially. Love, Jack." This, in so many words, is telling the consumer that Jack Daniels is here with them. Many other companies also chose to use this branding. Even the government has participated. The State of Ohio had a campaign running for \#inthistogetherohio, where famous figures around the state share that they are here for everyone.

Travel airlines have taken this concept even a step further. Not only do they show the consumer that we are in this together, but most of them are sharing their purpose behind the industry: you. American Airlines has extended its elite status program for an extra year because you are important. They have a commercial running with the campaign slogan, "You are why we fly." The video shows what American Airlines is doing to transport health professionals and medical supplies to keep you safe. Visually, it strongly parallels the campaign American Airlines from 9/11. Delta is running a campaign slogan "Our Promise: Your Safety Above All" and "Together as One." Delta is stating both messaging in their social media; they are also showing how they are transporting supplies.

\section{COVID-19 Post-Crisis Advertising Predictions}

Advertising Strategies

The major advertising strategies after COVID-19, specifically within travel, will largely mirror those of 9/11.

Promotion push instead of pull strategy. Although an unmeasurable bet, it is safe that emotions are higher right now than they have ever been as a collective nation. People are feeling all sorts of emotions in a combination that has not been felt before. There is fear for both the present and the future. There is fear over household finances. Greatest of all, there is fear and anxiety for the health and safety of oneself and one's families. Travel companies and destinations need to push the consumer and the traveler through their needs and wants. The advertiser needs to push the traveler past the psychological aftermath.

For the tourist traveler. To push the tourist to a new destination, advertisers will focus on the feelings that come with entertainment and localism. People are bored and unsettled. The population has been living in a state of temporary normal, and they will "want to get back to what is real." 19 The entertainment, the carefreeness, and the fun of travel will immediately appeal to them. We can expect to see new events, festivals, and "coffee shop passports" popping up. They needed a sense of community. A community that "demonstrates that visitors would be welcome, and serve as a guide to enjoying the available tourism product in that locality."18 This "meet[s] the social connectivity imperative and help[s] to build demand for local services that will in turn support supply-side development."18

For the corporate traveler. The corporate traveler advertising strategy will need to focus mainly on instilling confidence, safety, and customer satisfaction into the consumer. If they must travel, then each airline and hotel needs to differentiate themselves from the competition. Corporate travel will need to continue at some point, so it is up to each company's marketing teams to distinguish themselves. The corporate traveler will be looking for outstanding customer service and safety. They will also be looking to be persuaded of the need to travel over video conferencing. Advertising will have to showcase the distinct value to in-person business.

\section{Messaging}

Below are the six main advertisement messaging constructs that are predicted to come from COVID-19, specifically within the travel industry: 
American Dream. Messaging of the American Dream will come from both destinations and from travel companies. This will come in multiple forms. There will be new festivals, concerts, events, and challenges that boast of patriotism and city pride. There will be ads, specifically in airlines, which push the need to keep supporting America and using American companies to travel. There will be messaging and feelings of community and togetherness because "we," as America, got through it.

Confidence in safety. For airlines specifically, there will be many campaigns run around the concept that they have instituted more safety procedures and precautions. This has already been initiated. As seen above, many companies are pushing safety as the top priority and featuring masks in every image and video. Airlines will need to overcome the consumer fear and show that they will take care of them and that they will make sure safety in travel is their number one priority. We are in this together. This one will also strongly mirror 9/11 ads. If American Airlines used the exact same wording in a new commercial: "You knew you would be back, you'd all be back together. Somewhere in America." Would anyone be surprised? Verbiage and emotions are the same. These companies want the consumer to know that they are here to help and make everything as smooth and easy as possible. However, they also want to show the consumer that their priority lies within the individual's emotions and well-being.

Support local tourism. This has already become a theme among the nation during COVID-19 but will be vital to put into place regarding travel. People want to feel that they are welcome wherever; that they do not have to only stay in their space. The local community needs to be welcoming and to make people feel like their connectivity is desired. This will increase the demand for local businesses. People will want the chance to support local businesses and financially by experiencing their goods and services.

Connect with those you missed. The biggest emotional pull that will override the travel fear will connect with the people you missed. Much like the campaign from American Airlines after 9/11, there will be ploys to connect with family, friends, and nature. There will be narratives about the time wasted and the need that you have to connect. There will be information about the human need for connection.

Explore your city in a new way. Even though travel is normally thought of as adventure and abnormal, in contrast to the stay-at-home order, it will feel so exciting and desired that consumers will believe travel and freedom was always a part of what was real to them. The advertiser will need to push that emotion of fun enough so that the consumer can overcome the emotional fear of traveling and contracting COVID-19. For tourism in general, there needs to be a sense of normalcy and heightened normalcy or fun. People need a break and release from the emotional strains of the pandemic. There will be an increase in opportunity and demand. This is where tourism companies need to step in to create events, themes, and situations where the tourist can feel free and safe.

\section{Qualification}

COVID-19 is, in one sense, completely different from the attacks on 9/11. Economically the effects are already much larger:

Recent pandemics had markedly different infection patterns compared to COVID-19. Hence financial losses were comparatively low. In 2008, the World Bank predicted that the global cost of a bird-flu pandemic would be $3.1 \%$ of World GDP, which in 2019 was $\$ 88,081 \mathrm{~b}$ - resulting in an estimated 2019 cost of $\$ 2,732 \mathrm{~b}$. COVID-19 will generate a much more significant disruption than the World Bank's 2008 prediction, with the World Economic Forum (in March 2020) estimating that the cost of COVID-19 in 2020 will be at least US $\$ 1$ t.. ${ }^{1}$

However, because of the state of crisis and emotional similarities, as a marketer, we can take $9 / 11$ as a mere shadow of what will happen after COVID-19. 9/11 unified the United States, while in many ways, COVID-19 divided the nation.

\section{CONCLUSION}

The United States and the world have been through many crises over recent decades. There have been health pandemics, including SARS, H1N1, and Ebola outbreaks. There have been security crises like 9/11. In 2020, there was COVID-19. In these crises, the nation pulls together to support the American people and the American economy. For COVID-19, when life begins to emulate "normal," advertising will look a lot like it did after 9/11. There will be a push for consumers to reach over their emotional fears. There will be messaging to draw in consumers to the American dream, local tourism, see the world in new ways, and reconnect to their roots. 


\section{ACKNOWLEDGEMENTS}

Hannah thanks Dr. Allen Knight for being a constant encouragement in her academic success and for pushing her to be better, Dr. Caleb Chan for leading in a way that shows how to make Christ evident in a business career, her dad and family for encouraging her always to strive higher professionally, her mom for pushing her to consider COVID-19 as a research topic, Katie Warner for reading through every draft, and finally, her college roommate, Samantha Marchand, for listening to every new thesis idea before settling on this one.

\section{REFERENCES}

1. Evans, N., \& Elphick, S. (2005) Models of crisis management: an evaluation of their value for strategic planning in the international travel industry. International Journal of Tourism Research, 7(3), 135-150. doi: 10.1002/jtr.527

2. JeffCoxCNBCcom. (2020, July 30). Second-quarter GDP plunged by worst-ever 32.9\% amid virus-induced shutdown. Retrieved from https:// wmw.cnbc.com/2020/07/30/us-gdp-q2-2020-first-reading.html (accessed October 2020)

3. Johnson, B. (2020, March 17) Opinion: What $9 / 11$ can teach us about marketing in the time of coronavirus. Retrieved from bttps:// adage.com/article/cmo-strategy/opinion-what-911-can-teach-us-about-marketing-time-coronavirus/2244906 (accessed Mar 2020)

4. Microsoft: The Power of Teams. (2020, April 01). Retrieved from https:/ / www.adsoftheworld.com/ media/film/ microsoft_the_power_of_teams? className=collection-lb (accessed October 2020)

5. Mike Axisa \&. R.J. Anderson. (2020, September 15). MLB schedule has 43 total games postponed due to positive COVID19 cases. Retrieved from https:// wmw.cbssports.com/mlb/news/mlb-schedule-has-43-total-games-postponed-due-to-positive-covid-19-cases/ (accessed October 2020)

6. Pond, S. (2020, April 06). All the Awards Shows That Have Been Canceled, Postponed or Made Rule Changes Because of Coronavirus. Retrieved from https:/ / www.thewrap.com/ all-the-awards-shows-that-have-been-canceled-postponed-or-made-rule-changesbecause-of-coronavirus/ (accessed October 2020)

7. Brown, M. (2020, May 01). An Inside Look At The NFL's 2020 Virtual Draft Shows Elements Are Here To Stay. Retrieved from https:/ / www forbes.com/sites/maurybrown/2020/05/01/an-inside-look-at-the-nfls-2020-virtual-draft-shows-elements-are-here-to-stay/ (accessed October 2020)

8. Vary, A. (2020, August 06). With 'Mulan,' Disney Tests Out Entirely New Early VOD Model. Retrieved https:/ / variety.com/2020/film/news/mulan-disney-plus-premiere-1234711185/ (accessed October 2020)

9. Salcedo, A., Yar, S., \& Cherelus, G. (2020, March 15). Coronavirus Travel Restrictions, Across the Globe. Retrieved from https:/ / mww.nytimes.com/article/ coronavirus-travel-restrictions.html (accessed October 2020)

10. Prast, R. (2020, September 10). Update: Many 2020 Las Vegas conventions move to 2021, go virtual. from bttps:/ / wmw.reviewjournal.com/ business/conventions/ update-many-2020-las-vegas-conventions-move-to-2021-go-virtual-1989650/ (accessed October 2020)

11. Redaktion, O. (2020, April 21). Oktoberfest 2020 is cancelled: "It hurts!" Retrieved from https:// wmw.oktoberfest.de/ en/magazine/oktoberfest-news/2020/oktoberfest-2020-will-be-cancelled (accessed October 2020)

12. Coronavirus: Irish St Patrick's Day parades cancelled. (2020, March 09). Retrieved from bttps:// wmw.bbc.com/ news/world-europe51806551 (accessed October 2020

13. Perez, S. (2020, March 30). Videoconferencing apps saw a record 62M downloads during one week in March. Retrieved from https:// techcrunch.com/2020/03/30/video-conferencing-apps-saw-a-record-62m-downloads-during-one-week-in-march/ (accessed October 2020)

14. Argenti, P. A. (2019, January 04) Crisis Communication: Lessons from 9/11. Retrieved from https:// hbr.org/2002/12/crisiscommunication-lessons-from-911 (accessed Mar 2020)

15. Clark D.E., McGibany J.M., Myers A. (2009) The Effects of $9 / 11$ on the Airline Travel Industry. In: Morgan M.J. (eds.) The Impact of 9/11 on Business and Economics. The Day that Changed Everything?. Palgrave Macmillan, New York.

16. Dignam, C. (2013, July 23) Marketing After 9/11. Retrieved from bttps:// wmw.campaignlive.com/article/marketing-9-11/158038 (accessed Mar 2020)

17. Magazine, P. (2020, April 15). Coronavirus Will Change the World Permanently. Here's How. Retrieved from https://www.politico.com/news/magazine/2020/03/19/coronavirus-effect-economy-life-society-analysis-covid-13557 (accessed October 2020)

18. Peters, K., Peters, J., \& Peters, N. (2020) Visit People Tourism Recovery After Disaster. KPPM Strategy, 1-17.

19. Carden, A. R. (2005) The Use of Persuasive Appeals and Public Relations in the Travel and Tourism Industry Post-9/11. Journal of Hospitality \& Leisure Marketing, 12(1-2), 79-95. doi: 10.1300/j150v12n01_06

20. How COVID-19 Has-And Has Not-Affected Global Ad Spending. (n.d.) [Digital image]. Retrieved from https:// www.emarketer.com/ content/ how-coronavirus-affects-global-ad-spending (accessed Mar 2020)

21. Roper, W. (March 27, 2020) U.S. Ad Revenue Likely to Plummet [Digital image]. Retrieved May 09, 2020, from https:// www.statista.com/ chart/21271/ad-revenue-forecasts-after-coronavirus/ (accessed Mar 2020)

22. Gautier, A. (2002, April) The Business of Travel. N.Z. Marketing Magazine, 21(3), 35.

23. Klein, E. (2002, January 14) Better Days Ahead for the Local Travel Industry. Focus on Business and Personal Travel, pp. 11-12. 
24. Schwab, Kristin. “Advertisers Are Trying to Adapt to COVID-19.” Marketplace, 5 Apr. 2020, www.marketplace.org/2020/04/03/advertisers-are-trying-adapt-covid19/.

25. Mike Allen. (2010, May 19) American Airlines Post-9/11 Campaign. Retrieved from http://www.mikeallenwrites.com/americanairlines-post-911-campaign/(accessed April 2020)

26. TheWrap. (February 25, 2020) Leading cable news networks in the United States in February 2020, by number of total day viewers (in thousands) [Graph]. In Statista. Retrieved May 09, 2020, from https:// wmw.statista.com/statistics/409313/cable-newsnetwork-total-day-viewers-usa/ (accessed Mar 2020)

27. Ellefson, L. (2020, February 25) February Cable News Ratings: Fox News Posts Best Primetime Numbers Ever. Retrieved from https:/ / www.thewrap.com/february-cable-news-ratings-fox-news-posts-best-primetime-numbers-ever/ (accessed Mar 2020)

28. McAteer, O. (2020, March 27) Coke goes big with COVID social distancing in Times Square. Retrieved from bttps:/ / www.campaignlive.com/ article/ coke-goes-big-covid-social-distancing-times-square/1677909 (accessed April 2020)

29. Gartner, Inc. (n.d.) How Active Are Activewear Brands in the Fight against COVID-19. Retrieved from https:/ / wmw.gartner.com/ en/ marketing/insights/daily-insights/ how-active-are-activewear-brands-in-the-fight-against-covid-19 (accessed April 2020)

30. Dasgupta, S. (2020, April 18) Stay home: Honda promotes Covid-19 awareness with this homemade Civic commercial. Retrieved from https:/ / auto.hindustantimes.com/ auto/news/stay-home-honda-promotes-covid-19-awareness-with-this-homemade-civiccommercial-41587186158063.html (accessed April 2020)

31. Today Show. (2020, March 24). McDonald's gives its famous logo a makeover to promote social distancing. Retrieved from https:// www.today.com/food/ mcdonald-s-changes-golden-arches-logo-amid-coronavirus-outbreak-t176653 (accessed April 2020)

32. Perez, S. (2020, March 25) Spotify adds fundraising features and a COVID-19 news hub to address the health crisis. Retrieved from https: / techcrunch.com/2020/03/25/spotify-adds-fundraising-features-and-a-covid-19-news-bub-to-address-the-healthcrisis/(accessed April 2020)

33. American Airlines: You Are Why We Fly. (2020, April 01) Retrieved from https:/ / www.adsoftheworld.com/ media/ film/ american_airlines_you_are_why_we_fly?className =collectionlbecontentid=387208 Einline=true\#collection-lb-content (accessed April 2020)

34. Beer, J. (2020, March 17) Ford reads the culture right with new coronavirus response advertising. Retrieved from https:/ / www.fastcompany.com/90478322/ford-reads-the-culture-right-with-new-coronavirus-response-advertising

35. Beer, J. (2020, March 30) 'We're all in this together'? Why brands have so little to say in the pandemic. Retrieved from https:/ / www.fastcompany.com/90483063/were-all-in-this-together-why-brands-have-so-little-to-say-in-the-pandemic (accessed April 2020)

36. Albers-Miller, N. D., Straughan, R. D., \& Prenshaw, P. J. (2007) Advertising travel services to the business traveler. Tourism Management: Analysis, Behaviour and Strategy, 185-196. doi: 10.1079/9781845933234.0185

37. Austin, C. (2012, September 11) These Are The 10 Worst Ads Exploiting The 9/11 Attacks. Retrieved from bttps:/ / www.businessinsider.com/10-ads-exploiting-the-911-attacks-2012-9 (accessed Mar 2020)

38. Elkin, T. (n.d.) Travel Sites Bounce Back After 9/11. Advertising Age.

39. Fitzgerald, K. (n.d.) Travel Finds Its Fleet. Advertising Age.

\section{ABOUT STUDENT AUTHOR}

Hannah Gilliam is a recent graduate, May 2020 with a Major in Business Administration with a Marketing Concentration and a French Language Minor. Originally from Columbus, OH, she studied at Spring Arbor University, and throughout her four years worked several internships in Marketing. Her long-term goals include opening her own wedding and events planning company. After graduation she returned to Spring Arbor University to work as the Alumni and Student Relations Coordinator and is enjoying aiding in the development of her alma mater in new marketing strategies.

\section{PRESS SUMMARY}

COVID-19 has severely changed the world and the global economic market. These changes are comparable to the effects of 9/11; people are fearful, anxious, and the future is unknown. After 9/11 the tourism industry had to think quickly to reevaluate the consumer needs and market responses in order to push numbers back up. Because of the similar long-term crisis effects from COVID-19, we can compare the two events to run a predictive analysis on the market response and messaging that will arise in the travel industry. There will perhaps be a theme and direction for messaging within the travel industry post-COVID-19 based on the culture and spirit of The American Dream, confidence in safety, we're in this together, support local tourism, explore your city in a new way, and connect with those you missed. 
Print Edition ISSN 1536-4585

Online Edition ISSN 2375-8732 